\title{
Theory of Prehomogeneous Vector Spaces without Regularity Condition
}

\author{
In memory of N. Banba
}

By

\section{Akihiko GroJA*}

\section{§ 0. Introduction}

0.0. Let $G$ be a connected reductive group defined over the complex number field $\boldsymbol{C}$ and $(\rho, V)$ its finite dimensional rational representation. A triple $(G, \rho, V)$ is called a prehomogeneous vector space (abbrev. PV) if there exists a Zariski open dense orbit in $V$. A polynomial function $f(\neq 0)$ on $V$ is called a relative invariant of character $\phi$ if $f(g v)=\phi(g) f(v)$ for any $g \in G$ and $v \in V$.

0.1. Roughly speaking, a main result of the theory of prehomogeneous vector spaces [24], [28], [30] says that, if the regularity condition

$$
\operatorname{det}\left(\frac{\partial^{2} \log f}{\partial x_{i} \partial x_{j}}\right) \neq 0
$$

is satisfied, the Fourier transform of a complex power $f^{\alpha}$ is also a complex power $f^{-\infty}$ multiplied with certain factors. Thus it has been believed that, for an application of the theory of prehomogeneous vector spaces, it is necessary to assume the regularity condition.

0.2. A prehomogeneous vector space is called regular if it has a relative invariant satisfying the regularity condition. By [27; \$4] and [22], the following conditions for a prehomogeneous vector space $(G, \rho, V)$ are equivalent to each other:

(1) $(G, \rho, V)$ is regular.

(2) The isotropy subgroup of $G$ at a point in the open orbit of $V$ is reduc-

Communicated by K. Saito, October 16, 1990. Revised April 11, 1991.

1991 Mathematics Subject Classification: 14L30

* Institute of Mathematics, Yoshida College, Kyoto University, Kyoto 606-01, Japan. 
tive.

(3) $([G, G], \rho, V)$ is stable.

(A $G$-module $V$ is said to be stable if there is an open dense subset of $V$ consisting of $G$-orbits which are closed in $V_{\text {.) }}$

0.3. The classification theory of prehomogeneous vector spaces presents a strange contrast to the application of the theory; once we assume the regularity, classification results usually become much more complicated. For example, assume the regularity in the first part of the theorem in $[7 ; 1.13]$. It would be worth noting that, in the classification theory of representations of reductive groups satisfying some properties other than prehomogeneity, analogous phenomenon occurs; once we assume the stability, classification results become complicated. For example, assume the stability in the second part of the theorem in $[7 ; 1.13]$.

0.4. Prehomogeneous vector spaces also appear in the Dynkin-Kostant theory concerning the nilpotent orbits of complex semisimple Lie algebras ([31]), which plays an important role in the representation theory. See [18]. The prehomogeneous vector spaces appearing in the Dynkin-Kostant theory form a class $(D K)$, which is contained in the class $(\mathbb{R})$ of regular prehomogeneous vector spaces. Denote the totality of PV's by (PV). Among these three classes $(D K) \subset(R) \subset(P V)$, the author considers that $(D K)$ forms a natural class, since it has a natural characterization [7]. If we restrict ourselves to the case where $[G, G]$ is simple and $V$ is irreducible, $(P V)$ is a class which 'corresponds" to the class of Kac-Moody Lie algebras of finite type as is indicated in [7;1.13], neglecting a small deviation. (Similarly, the class of coregular $(G, \rho, V)$ 's 'cor-

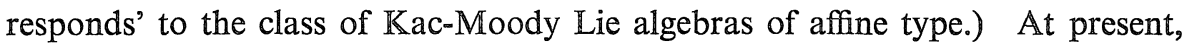
the author can not find a proper place for $(R)$ in the classification theory.

0.5. Without assuming $G$ to be reductive, it is possible to define the regularity of $(G, \rho, V)$ by the existence of a relative invariant satisfying the regularity condition. But it seems to the author that there is a room for doubt about the naturality of this concept.

First, it was conjectured that, for a regular $(G, \rho, V)$ in the generalized sense, the open $G$-orbit in $V$ is an affine variety. But, recently this conjecture turned out to be false [6].

Secondly, as is shown by Rubenthaler-Schiffmann [23] and Bopp-Rubenthaler [2], some examples of regular PV's in the generalized sense can be treated 
and even better understood in the framework of PV's with reductive $G$. See also [8].

0.6. By these reasons, we study prehomogeneous vector spaces assuming the reductivity of $G$ and without assuming the regularity. Roughly speaking, our main result is that, even without assuming the regularity condition, the Fourier transform of a complex power $f^{\alpha}$ of a relative invariant $f$ is also a complex power $f^{-\alpha}$ multiplied with a certain factor $h$. Unlike the regular case, $h$ is supported by a closure of an orbit whose dimension is, in general, not equal to $\operatorname{dim} V$. This phenomenon seems to fit naturally to a more general scheme including the differential equations of Harish-Chandra [12].

0.7. In our study of prehomogeneous vector spaces, we constantly rely on the theory of $D$-modules, which is mainly developed by M. Sato, T. Kawai and M. Kashiwara. In this regard, see (4.21).

0.8. This paper consists of four sections. In the first section we study geometric structure of prehomogeneous vector spaces. The main result of this section is (1.18). In the second section, we review the theory of systems of linear differential equations. In the third section, we study a system of linear differential equations $D f^{\alpha}$ satisfied by $f^{\alpha}$. The purpose of this section is to determine the Fourier transform of $D f^{\alpha}$ and the sheaf of its holomorphic solutions. The main results are (3.11) and (3.23). In the fourth section, we study the hyperfunction solutions of $D f^{\alpha}$ and their Fourier transforms. The main result is (4.19).

0.9. The present paper is partly based on the series of lectures given at Tsukuba University in October 1988. The author would like to express his thanks to those who attended at the lectures, in particular to Prof. T. Kimura. The author is profitted much from the note [9] taken by Prof. T. Kimura and his comments. The author would like to thank Prof. M. Muro and Prof. R. Hotta for their careful reading and comments, which helped the author to improve the paper to a great extent. It is a great pleasure to acknowledge the valuable comments of the referee, by which the author could correct several errors contained in the original manuscript.

\section{$\S$ 1. Structure of Prehomogeneous Vector Spaces}

1.0. The purpose of this section is to prove (1.18), which describes a 
geometric structure of a prehomogeneous vector space. Although (1.3) and (1.5)-(1.9) are contained in [27], we include them here for the sake of the convenience of the readers.

Defimition 1.1. Let $G$ be a connected reductive group defined over the complex number field $\mathbb{C}$ and $\rho: G \rightarrow G L(V)$ a finite dimensional rational representation. Such a triple $(G, \rho, V)$ is called a prehomogeneous vector space (abbrev. PV) if there exists an open dense $G$-orbit, say $O_{0}=G \circ v_{0}$, in $V$.

Definimitom 1.2. Let $f$ be a polynomial function on $V$ which is not identically zero and $\phi \in \operatorname{Hom}\left(G, \mathbb{C}^{\times}\right)$, where $\mathbb{C}^{\times}=G L_{1}(\mathbb{C})$. If $f(g v)=\phi(g) f(v)$ for any $g \in G$ and $v \in V$, then we call $f$ a relative invariant and $\phi$ the character of $f$.

Lemma 1.3. Let $(G, \rho, V)$ be a prehomogeneous vector space.

(1) If two relative invariants $f_{1}$ and $f_{2}$ have the same character, then $f_{1}$ is a constant multiple of $f_{2}$.

(2) A relative invariant is homogeneous.

Proof. (1) Let $f=f_{1} / f_{2}$. Then $f$ is constant on $O_{0}=G \circ v_{0}$, which is open and dense in $V$. Hence $f$ is a constant.

(2) For $c \in \mathbb{C}^{\times}$, let $f_{c}(v)=f(c v)$. Then $f$ and $f_{c}$ have the same character. Hence $f_{c}$ is a constant multiple of $f$.

Henceforth we fix a prehomogeneous vector space $(G, \rho, V)$ and a relatively invariant polynomial function $f$ on $V$ with character $\phi$. Let $n=\operatorname{dim} V, d=\operatorname{deg} f$ and $\Omega=\Omega(f)=f^{-1}\left(\mathbb{C}^{\times}\right)$. Note that the open orbit $O_{0}$ is contained in $\Omega$.

Lemma 1.4. (1) There is a unique G-orbit $O_{1}=O_{1}(f)$ in $\Omega=\Omega(f)$ which is Zariski closed in $\Omega$.

(2) For $c \in \mathbb{C}^{\times}$and $v \in O_{1}, c v \in O_{1}$.

Proof. (1) Let $\pi: \Omega \rightarrow \Omega / G$ be the projection onto the quotient space. Since the Zariski closure $\bar{O}_{0}$ in $\Omega$ is equal to $\Omega$, we get $\pi(\Omega)=\pi\left(\overline{O_{0}}\right) \subset \overline{\pi\left(O_{0}\right)}$. Hence $\Omega / G$ consists of only one point. Since the (closed) points of the quotient space are in one-to-one correspondence with the closed orbits [21], $\Omega$ has a unique closed $G$-orbit.

(2) Since $c O_{1}$ is also a closed $G$-orbit of $\Omega, c O_{1}=O_{1}$.

Lemma 1.5 . Let $\rho^{\vee}: G \rightarrow G L\left(V^{\vee}\right)$ be a rational representation and $\langle 1\rangle$ : $V^{\vee} \times V \rightarrow \mathbb{C}$ a non-degenerate bilinear form such that $\left\langle g v^{\vee} \mid g v\right\rangle=\left\langle v^{\vee} \mid v\right\rangle$ for any $g \in G, v^{\vee} \in V^{\vee}$ and $v \in V$. 
(1) The triple $\left(G, \rho^{\vee}, V^{\vee}\right)$ is a prehomogeneous vector space.

(2) The prehomogeneous vector space $\left(G, \rho^{\vee}, V^{\vee}\right)$ has a relatively invariant polynomial whose character is $\phi^{-1}$, and whose polynomial degree is equal to $d(=\operatorname{deg} f)$.

Proof. (1) Let $K$ be a maximal compact subgroup of $G$. If we fix a basis of $V$, we can identify $G L(V)$ with $G L_{n}(\mathbb{C})$ and $V$ with $\mathbb{C}^{n}$. The compact subgroup $\rho(K)$ of $G L_{n}(C)$ is contained in a conjugate of the unitary group. Hence by taking a vector basis $\left\{v_{1}, \cdots, v_{n}\right\}$ of $V$ suitably, we may assume that $\rho(K)$ is contained in the unitary group. Let $\left\{v_{1}^{\vee}, \cdots, v_{n}^{\vee}\right\}$ be the dual basis of $V^{\vee}$. By this basis, we identify $G L\left(V^{\vee}\right)$ with $G L_{n}(\mathbb{C})$ and $V^{\vee}$ with $\mathbb{C}^{n}$. Then $\rho^{\vee}(k)=\rho(k)^{*-1}=\rho(k)^{c}$ for any $k \in K$. Here * means the transposition of a matrix and ${ }^{c}$ means the complex conjugation. Hence $\rho^{v}(K) \cdot v_{0}^{c}=\left(\rho(K) v_{0}\right)^{c}$ is Zariski dense in the orbit $\left(\rho(G) v_{0}\right)^{c}=O_{0}^{c}$. Hence $\rho^{\vee}(G) v_{0}^{c}$ is Zariski dense in $V^{\vee}$.

(2) Let $f^{\vee}$ be a polynomial function on $V^{\vee}$ defined by $f^{\vee}\left(v^{\vee}\right)=f\left(v^{\vee c}\right)^{c}$ for any $v^{\vee} \in V^{\vee}=\mathbb{C}^{n}$. Then

$$
f^{\vee}\left(\rho^{\vee}(k) v^{\vee}\right)=f\left(\rho(k) v^{\vee c}\right)^{c}=\phi(k)^{c} f\left(v^{\vee c}\right)^{c}=\phi(k)^{-1} f^{\vee}\left(v^{\vee}\right)
$$

for any $k \in K$ and $v^{\vee} \in V^{\vee}$. Since $K$ is Zariski dense in $G$,

$$
f^{\vee}\left(\rho^{\vee}(g) v^{\vee}\right)=\phi(g)^{-1} f^{\vee}\left(v^{\vee}\right)
$$

for any $g \in G$ and $v^{\vee} \in V^{\vee}$. Since any relative invariant of $\left(G, \rho^{\vee}, V^{\vee}\right)$ with character $\phi^{-1}$ is a constant multiple of this particular $f^{\vee}$, it is of degree $d$.

Henceforth we fix $\left(G, \rho^{\vee}, V^{\vee}\right),\langle 1\rangle: V^{\vee} \times V \rightarrow \mathbb{C},\left\{v_{1}, \cdots, v_{n}\right\}$, and $\left\{v_{1}^{\vee}, \cdots\right.$, $\left.v_{n}^{\vee}\right\}$ as in the above lemma. We also fix a relative invariant $f^{\vee}$ on $V^{\vee}$ whose character is $\phi^{-1}$. By convention, we set $\left\langle v^{v} \mid v\right\rangle=\left\langle v \mid v^{v}\right\rangle$. Let $\Omega^{v}=\Omega^{v}\left(f^{v}\right)$ $=f^{\vee-1}\left(C^{\times}\right)$and $O_{0}^{\vee}$ (resp. $\left.O_{1}^{\vee}=O_{1}^{\vee}\left(f^{\vee}\right)\right)$ be the unique open (resp. closed) $G$-orbit in $\Omega^{\mathrm{v}}$.

Lemma 1.6. There exists a polynomial $b(s)=b_{0} s^{d}+b_{1} s^{d-1}+\cdots+b_{d} \in C[s]$ of degree at most $d$ such that both of the formal identities

$$
f^{\vee}\left(\operatorname{grad}_{x}\right) f(x)^{s+1}=b(s) f(x)^{s}
$$

and

$$
f\left(\operatorname{grad}_{y}\right) f^{\vee}(y)^{s+1}=b(s) f^{\vee}(y)^{s}
$$

hold in the same time. Here $\operatorname{grad}_{x}=\left(\frac{\partial}{\partial x_{1}}, \cdots, \frac{\partial}{\partial x_{n}}\right)$ and $\operatorname{grad}_{y}=\left(\frac{\partial}{\partial y_{1}}, \cdots, \frac{\partial}{\partial y_{n}}\right)$. 
(Sometimes, we write grad for $\operatorname{grad}_{x}$ or $\operatorname{grad}_{y^{*}}$ )

Proof. In order to prove the first identity, we may assume $s$ to be a natural number. By $(1.3,(1))$,

$$
f^{\vee}\left(\operatorname{grad}_{x}\right) f(x)^{s+1}=b(s) f(x)^{s}
$$

with some constant $b(s)$ which depends on $s$ but not on $x$. Obviously $b(s)$ is a polynomial in $s$. By the same argument, we get a similar identity

$$
f\left(\operatorname{grad}_{y}\right) f^{\vee}(y)^{s+1}=b^{\vee}(s) f^{\vee}(y)^{s}
$$

with some polynomial $b^{\vee}(s) \in \mathbb{C}[s]$. First, let us take as $f^{\vee}$ the polynomial constructed in the proof of $(1.5,(2))$. Then we can write $f(x)=\sum f_{i_{1}, \cdots, i_{n}} x_{1}^{i_{1} \cdots \cdots} x_{n}^{i_{n}}$

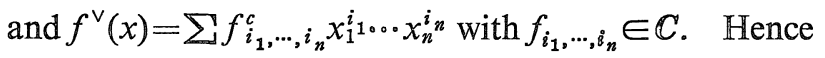

$$
b(0)=f^{\vee}(\operatorname{grad}) f(x)=\sum\left|f_{i_{1}, \cdots, i_{n}}\right|^{2} i_{1} ! \circ \cdots i_{n} !=f(\operatorname{grad}) f^{\vee}(y)=b^{\vee}(0)
$$

is a positive real number. Similarly

$$
\begin{gathered}
b(0) b(1) \cdots b(m-1)=f^{\vee}(\mathrm{grad})^{m} f(x)^{m}=f(\mathrm{grad})^{m} f^{\vee}(x)^{m} \\
=b^{\vee}(0) b^{\vee}(1) \cdots b^{\vee}(m-1)>0 .
\end{gathered}
$$

Hence $b^{\vee}(s)=b(s)$ for our special choice of $f^{\vee}$. If we replace $f^{\vee}$ by $c f^{\vee}(c \in$ $\left.\mathbb{C}^{\times}\right)$, then $b(s)$ and $b^{\vee}(s)$ are replaced by $c b(s)$ and $c b^{\vee}(s)$, respectively. Hence $b^{\vee}(s)=b(s)$ for any relative invariant $f^{\vee}$.

Lemma 1.7。 $b_{0} \neq 0$, i.e., $\operatorname{deg} b(s)=d$.

Proof. We may assume that $f(x)=\sum f_{i_{1}, \cdots, i_{n}} x_{1}^{i_{1} \cdots x_{n}} x_{n}^{i_{n}}$ and $f^{\vee}(x)=$

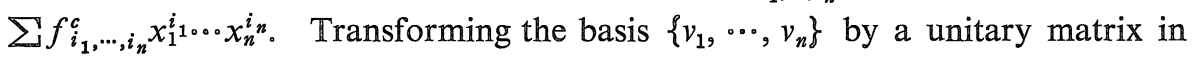
$G L_{n}(\mathbb{C})$, we may assume that $f(1,0, \cdots, 0) \neq 0$, i.e., $f_{d, 0, \cdots, 0} \neq 0$. If $\operatorname{deg} b(s)=$ $e(\leq d)$, then there exists a constant $c$ such that $|b(s)| \leq c(s+1)^{e}$ for $s \geq 0$. Then

$$
\left|f_{d, 0, \cdots, 0}\right|^{2 m}(m d) ! \leq f^{\vee}(\mathrm{grad})^{m} f(x)^{m}=b(0) b(1) \cdots b(m-1) \leq c^{m}(m !)^{e} .
$$

Since $(m d) ! /(m !)^{d} \geq 1,(m !)^{d-e} \leq\left(c /\left|f_{d, 0, \cdots, 0}\right|^{2}\right)^{m}$. Hence $d=e$.

Lemminas 1.8. Let $F=\operatorname{grad} \log f$ and $F^{\vee}=\operatorname{grad} \log f^{\vee}$.

(1) For $v \in \Omega, f^{\vee}(F(v))=b_{0} f(v)^{-1}$. (Recall that $\Omega=f^{-1} \mathbb{C}^{\times}$.)

(2) For $v^{\vee} \in \Omega^{\vee}, f\left(F^{\vee}\left(v^{\vee}\right)\right)=b_{0} f^{\vee}\left(v^{\vee}\right)^{-1}$.

(3) $F$ defines a regular morphism $\Omega \rightarrow \Omega^{\vee}$, compatible with G-actions.

(4) $F^{\vee}$ defines a regular morphism $\Omega^{\vee} \rightarrow \Omega$, compatible with G-actions.

(5) For $c \in \mathbb{C}^{\times}$and $v \in \Omega, F(c v)=c^{-1} F(v)$.

(6) For $c \in \mathbb{C}^{\times}$and $v^{\vee} \in \Omega^{\vee}, F^{\vee}\left(c v^{\vee}\right)=c^{-1} F^{\vee}\left(v^{\vee}\right)$. 
Proof. By (1.7), it is enough to prove the first assertion. If $i_{1}+\cdots+i_{n}=d$,

$$
\left(\frac{\partial}{\partial x_{1}}\right)^{i_{1}} \cdots\left(\frac{\partial}{\partial x_{n}}\right)^{i_{n}} f(x)^{s+1}=\left(s^{d}\left(\frac{\partial f}{\partial x_{1}}\right)^{i_{1}} \cdots\left(\frac{\partial f}{\partial x_{n}}\right)^{i_{n}}+O\left(s^{d-1}\right)\right) f(x)^{s+1-d} .
$$

Hence

$$
\begin{aligned}
f^{\vee}(\operatorname{grad}) f(x)^{s+1} & =\left(s^{d} f^{\vee}(\operatorname{grad} f)+O\left(s^{d-1}\right)\right) f(x)^{s+1-d} \\
= & \left(b_{0} s^{d}+O\left(s^{d-1}\right)\right) f(x)^{s} .
\end{aligned}
$$

Comparing the coefficients of $s^{d}$, we get the assertion.

The character $\phi: G \rightarrow C^{\times}$induces the character of $\operatorname{Lie}(G)$, which we shall denote by the same letter $\phi$.

Lemma 1.9. (1) For $v \in \Omega$ and $A \in \operatorname{Lie}(G)$,

$$
\langle F(v) \mid A v\rangle=\phi(A) .
$$

(2) For $v^{\vee} \in \Omega^{\vee}$ and $A \in \operatorname{Lie}(G)$,

$$
\left\langle F^{\vee}\left(v^{\vee}\right) \mid A v^{\vee}\right\rangle=-\phi(A) .
$$

Proof. If $\rho(A)=\left(a_{i j}\right)_{1 \leq i, j \leq n}$, then by differentiating $f(\exp (t A) \cdot v)=\phi(\exp$ $(t A)) f(v)$ at $t=0$, we get

$$
\left(\sum_{i, j=1}^{n} a_{i j} x_{j} \frac{\partial}{\partial x_{i}}-\phi(A)\right) f=0,
$$

which is nothing but the first assertion. In the same way, we get

$$
\left(\sum_{i, j=1}^{n}\left(-a_{j i}\right) y_{j} \frac{\partial}{\partial y_{i}}+\phi(A)\right) f^{\vee}=0
$$

and the second assertion.

Lemma 1.10. (1) For $v \in \Omega$ and $A \in \operatorname{Lie}(G)$,

$$
\left\langle F^{\vee} F(v)-v \mid A F(v)\right\rangle=0 \text {. }
$$

(2) $F\left(O_{0}\right)$ is an open dense subset of the Zariski closure $\overline{F(\Omega)}$ of $F(\Omega)$ in $\Omega^{\vee}$. (Recall that $F(\Omega) \subset \Omega^{\vee}$ by $(1.8,(3))$.

(3) If $v \in \Omega$ and $F(v) \in F\left(O_{0}\right)$, then

$$
F^{\vee} F(v)-v \in\left(T_{F(v)} F\left(O_{0}\right)\right)^{\perp} .
$$

Here $T_{p} X$ denotes the tangent space of $X$ at $p$, and $\left(T_{F(v)}\left(F\left(O_{0}\right)\right)^{\perp}\right.$ denotes the orthogonal complement in $V$ of $T_{F(v)} F\left(O_{0}\right) \subset T_{F(v)} V^{\vee}=V^{\vee}$. 
Proof. (1) By $(1.8,(3))$ and $(1.9,(2))$,

$$
\left\langle F^{\vee} F(v) \mid A \cdot F(v)\right\rangle=-\phi(A)
$$

for $v \in \Omega$ and $A \in \operatorname{Lie}(G)$. On the other hand, by $(1.9,(1))$,

$$
\langle v \mid A \cdot F(v)\rangle=-\langle A v \mid F(v)\rangle=-\phi(A) \text {. }
$$

By these equalities, we get the assertion.

(2) Since $F\left(O_{0}\right)$ is Zariski dense in $\overline{F(\Omega)}$, it contains a non-empty open set of $\overline{F(\Omega)}$ by the Hilbert's second theorem (cf. $[32 ; 1.13]$ ). Since $F\left(O_{0}\right)$ is $G$ homogeneous, it is open in $\overline{F(\Omega)}$.

(3) $\mathrm{By}(1)$

$$
F^{\vee} F(v)-v \in(\operatorname{Lie}(G) \cdot F(v))^{\perp} .
$$

Since $F(v)=F\left(v^{\prime}\right)$ for some $v^{\prime} \in O_{0}$ by the assumption,

$$
\operatorname{Lie}(G) \cdot F(v)=T_{F(v)}\left(G \cdot F\left(v^{\prime}\right)\right)=T_{F(v)} F\left(O_{0}\right) .
$$

By (1.10.1) and (1.10.2), we get the assertion.

Lempmana H. $_{1}$. (1) Let $F(\Omega)_{0}$ be the union of all the open sets of $\overline{F(\Omega)}$ contained in $F(\Omega)$. If a point $v \in \Omega$ satisfies

$$
F(v) \in F(\Omega)_{0} \text { and }(d F)_{v}(V)=T_{F(v)} F(\Omega)_{0},
$$

then

$$
T_{v}\left(F^{-1} F(v)\right)=\left(T_{F(v)} F(\Omega)_{0}\right)^{\perp} .
$$

(For the moment, $F^{-1} F(v)$ and $T_{v}\left(F^{-1} F(v)\right)$ should be understood scheme theoretically.)

(2) If $v \in O_{0},(1.11 .1)$ is satisfied.

Proof. (1) The following conditions for $a=\left(a_{1}, \cdots, a_{n}\right)$ are equivalent:

(a) $a \in \mathbb{T}_{v}\left(F^{-1} F(v)\right)$.

(b) $(d F)_{v}(a)=0$.

(c) $\sum_{j=1}^{n} \frac{\partial^{2} \log f(v)}{\partial x_{i} \partial x_{j}} a_{j}=0$ for any $i$.

(d) $\sum_{i, j=1}^{n} b_{\dot{i}} \frac{\partial^{2} \log f(v)}{\partial x_{\dot{j}} \partial x_{j}} a_{j}=0$ for any $\left(b_{1}, \cdots, b_{n}\right)$.

(e) $(d F)_{v}(V) \perp a$.

(f) $T_{F(v)} F(\Omega)_{0} \perp a$. 
(2) Since the natural morphism $G \rightarrow G \cdot F(v)$ is a submersion, $F: G \cdot v \rightarrow$ $G \cdot F(v)$ is also a submersion. Hence if $v \in O_{0}$, by $(1.10,(2))$,

$$
T_{F(v)} F(\Omega)_{0}=T_{F(v)} F\left(O_{0}\right)=(d F)_{v}\left(T_{v} O_{0}\right)=(d F)_{v}(V) .
$$

Lemma 1.12. The morphism $F: O_{0} \rightarrow F\left(O_{0}\right)$ is smooth.

Proof. Since $O_{0}$ is $G$-homogeneous and $F$ is compatible with $G$-actions, it is enough to note that there is a point $v \in O_{0}$ such that $F$ is smooth at $v$.

Lemma 1.13. For $v \in O_{0}$,

$$
F^{-1} F(v) \supset v+\left(T_{F(v)} F\left(O_{0}\right)\right)^{\perp} .
$$

Proof. By (1.11), for a given $v \in O_{0}$, the tangent space of $O_{0} \cap F^{-1} F(v)$ at any point is the same space $\left(T_{F(v)} F(\Omega)_{0}\right)^{\perp}=\left(T_{F(v)} F\left(O_{0}\right)\right)^{-}$. By (1.12), $O_{0} \cap$ $F^{-1} F(v)$ is a union of open subsets of affine subspaces of $V$ which are parallel to $\left(T_{F(v)} F\left(O_{0}\right)\right)^{\perp}$. Thus we can find a non-empty open subset $U$ of $\left(T_{F(v)} F\left(O_{0}\right)\right)^{\perp}$ such that

$$
O_{0} \cap F^{-1} F(v) \supset v+U .
$$

If $v^{\prime} \in F^{-1} F(v)$, then by (1.8),

$$
b_{0} f\left(v^{\prime}\right)^{-1}=f^{\vee}\left(F\left(v^{\prime}\right)\right)=f^{\vee}(F(v))=b_{0} f(v)^{-1} .
$$

Hence, by (1.7), $f \equiv c$ on $F^{-1} F(v)$ for some non-zero constant $c$, i.e., $f^{-1}(c)$ $\supset F^{-1} F(v)$. Since $F^{-1} F(v)$ is closed in $\Omega$, it is also closed in $f^{-1}(c)$, which implies that $F^{-1} F(v)$ is closed in $V$. By (1.13.1), $F^{-1} F(v)$ contains the Zariski closure of $v+U$ in $V$, which is $v+\left(T_{F(v)} F\left(O_{0}\right)\right)^{\perp}$.

Lemma 1.14. The morphisms $F^{\vee}: \overline{F(\Omega)} \rightarrow \overline{F^{\vee}\left(\Omega^{\vee}\right)}$ and $F: \overline{F^{\vee}\left(\Omega^{\vee}\right)} \rightarrow \overline{F(\Omega)}$ are isomorphisms and the inverse of each other. (Here $\overline{F(\Omega)}\left(\right.$ resp. $\left.\overline{F^{\vee}\left(\Omega^{\vee}\right)}\right)$ is the Zariski closure in $\Omega^{\vee}($ resp. $\Omega)$.)

Proof. For any $v \in O_{0}$,

$$
F^{\vee} F(v) \in v+\left(T_{F(v)} F\left(O_{0}\right)\right)^{\perp} \subset F^{-1} F(v)
$$

by $(1.10,(3))$ and (1.13). Hence $F F^{\vee} F \equiv F$ on $O_{0}$, i.e., $F F^{\vee}$ is the identity on $F\left(O_{0}\right)$. Since $F\left(O_{0}\right)$ is dense in $\overline{F(\Omega)}, F F^{\vee}$ is the identity on $\overline{F(\Omega)}$. Analogously, $F^{\vee} F$ is the identity on $\overline{F^{\vee}\left(\Omega^{\vee}\right)}$.

Lemma 1.15. (1) $F(\Omega)$ is a smooth affine variety and Zariski closed in $\Omega^{\vee}$.

(2) For $v^{\vee} \in F(\Omega)$, 


$$
T_{v} v F(\Omega)=(d F)_{F^{v}\left(v^{v}\right)}(V)=(d F)_{F^{v}\left(v^{v}\right)}\left(T_{F^{v}\left(v^{v}\right)} F^{\vee}\left(\Omega^{v}\right)\right)
$$

and

$$
T_{F^{\vee}\left(v^{\vee}\right)}\left(F^{\vee}\left(\Omega^{\vee}\right)\right)=\left(d F^{\vee}\right)_{v^{\vee}}\left(V^{\vee}\right)=\left(d F^{\vee}\right)_{v} v\left(T_{v} \vee F(\Omega)\right) .
$$

Proof. By (1.14) and $(1.8,(4))$,

$$
F(\Omega)=\left\{v^{\vee} \in \Omega^{\vee} \mid v^{\vee}=F F^{\vee}\left(v^{\vee}\right)\right\} .
$$

Hence $F(\Omega)$ is closed in $\Omega^{\vee}$. Similarly, $\overline{F^{\vee}\left(\Omega^{\vee}\right)}=F^{\vee}\left(\Omega^{\vee}\right)$. As $F(\Omega)$ is a closed subset of the affine variety $\Omega^{\vee}=f^{\vee-1}\left(C^{\times}\right), F(\Omega)$ is an affine variety. By (1.14),

$$
\begin{aligned}
& T_{v^{v}} F(\Omega)=T_{F F^{v}\left(v^{v}\right)} F(\Omega) \supset(d F)_{F^{v}\left(v^{v}\right)}(V) \\
& \quad \supset(d F)_{F^{v}\left(v^{v}\right)}\left(T_{F^{v}\left(v^{v}\right)} F^{\vee}\left(\Omega^{v}\right)\right)=T_{F F^{v}\left(v^{v}\right)} F(\Omega)
\end{aligned}
$$

for $v^{v} \in F(\Omega)$. By these relations, we get the first equalities in (2). The second equalities can be proved in the same way. Let $a\left(v^{\vee}\right)=\operatorname{dim} T_{v} F(\Omega)$. Then $a$ is upper semicontinuous on $F(\Omega)$, i.e., the values of $a$ are not larger than $a\left(v^{v}\right)$ in a small neighbourhood of $v^{v}$. On the other hand, as $a\left(v^{v}\right)$ is the rank of $(d F)_{F^{\vee}\left(v^{v}\right)}$, it is lower semicontinuous. Hence $a$ is locally constant, and $F(\Omega)$ is smooth.

Lemma 1.16. For $v^{\vee} \in F(\Omega)$, the symmetric matrix

$$
\left(\frac{\partial^{2} \log f^{\vee}}{\partial y_{i} \partial y_{j}}\left(v^{\vee}\right)\right)_{1 \leq i, j \leq n}
$$

defines a $G_{v}$-invariant, non-degenerate, symmetric, bilinear form $B_{v} \vee$ on $T_{v} \vee F(\Omega)$. Here $G_{v}$ denotes the isotropy subgroup of $G$ at $v^{v}$.

Proof. If $g v_{i^{\prime}}=\sum_{i=1}^{n} v_{i} g_{i i^{\prime}}$, then $g^{-1} v_{i}^{\vee}=\sum_{i^{\prime}=1}^{n} g_{i i^{\prime}} v_{i^{\prime}}^{\vee}$ and

$$
\left(\frac{\partial^{2} \log f^{\vee}\left(g^{-1} \circ\right)}{\partial y_{i} \partial y_{j}}\right)_{i, j}=\left(g_{i i^{\prime}}\right)\left(\left(\frac{\partial^{2} \log f^{\vee}}{\partial y_{i^{\prime}} \partial y_{j^{\prime}}}\right)\left(g^{-1_{\circ}}\right)\right)\left(g_{j^{\prime}}\right)^{*} \text {. }
$$

Hence the bilinear form $B_{v^{v}}$ on $T_{v} v(\Omega)$ is $G_{v}$-invariant. Since $B_{v}$ is also given by

$$
T_{v} \vee F(\Omega) \times T_{v} \vee F(\Omega) \ni(a, b) \rightarrow\left\langle a \mid\left(d F^{\vee}\right)_{v} \vee(b)\right\rangle,
$$

its kernel is

$$
T_{v} \vee F(\Omega) \cap\left(\left(d F^{\vee}\right)_{v} v\left(T_{v} \vee F \Omega\right)\right)^{\perp},
$$

which is, by (1.11) and $(1.15,(2))$, equal to

$$
T_{v} \vee F(\Omega) \cap\left(T_{F^{\vee}\left(v^{\vee}\right)} F^{\vee}\left(\Omega^{\vee}\right)\right)^{\perp}=T_{v} F(\Omega) \cap T_{v}\left(F^{\vee-1} F^{\vee}\left(v^{\vee}\right)\right) .
$$


(Note that, by (1.14) and (1.15), $F(\Omega)_{0}=F(\Omega)$ and (1.11.1) is automatically satisfied.) Since $F(\Omega)$ is a cross section of $F^{\vee}: \Omega^{\vee} \rightarrow F^{\vee}\left(\Omega^{\vee}\right)$ by (1.14) and (1.15), and $F^{\vee-1} F^{\vee}\left(v^{\vee}\right)$ is a fibre,

$$
T_{v} F(\Omega) \cap T_{v \vee}\left(F^{\vee-1} F^{\vee}\left(v^{\vee}\right)\right)=0 .
$$

Hence $B_{v} v$ is non-degenerate.

Lemma 1.17 ([19]). Let $X$ be a smooth affine variety and $G$ a reductive group acting on $X$. Suppose that a $G_{x}$-invariant, non-degenerate, symmetric bilinear form on $T_{x} X$ is given for each point $x \in X$. Then there exists an open, dense, $G$-stable subset $U$ of $X$ such that $G \cdot x$ is closed in $X$ for any point $x \in U$.

Theorem 1.18. (1) The G-orbit $O_{1}$ is affine variety and the isotropy group at a point of $O_{1}$ is reductive.

(2) $F(\Omega)=F\left(O_{0}\right)=O_{1}^{v}$.

(3) The morphisms $F: O_{1} \rightarrow O_{1}^{\vee}$ and $F^{\vee}: O_{1}^{\vee} \rightarrow O_{1}$ are isomorphisms and the inverse morphisms of each other.

(4) Let $\left(T O_{1}^{\vee}\right)^{\perp}$ be the conormal bundle of $O_{1}^{\vee}$, i.e.,

$$
\left(T O_{1}^{\vee}\right)^{\perp}=\left\{\left(v, v^{\vee}\right) \in V \times O_{1}^{\vee} \mid v \in\left(T_{v} \vee O_{1}^{\vee}\right)^{\perp}\right\} .
$$

Let

$$
\Phi\left(v, v^{\vee}\right)=v+F^{\vee}\left(v^{\vee}\right) \quad \text { for }\left(v, v^{\vee}\right) \in\left(T O_{1}^{\vee}\right)^{\perp} .
$$

Then $\Phi$ defines a G-isomorphism between fibre spaces $\pi:\left(T O_{1}^{\vee}\right)^{\perp} \rightarrow O_{1}^{\vee}$ (natural projection) and $F: \Omega \rightarrow O_{1}^{\vee}$. The inverse of $\Phi$ is given by

$$
\Psi(v)=\left(v-F^{\vee} F(v), F(v)\right) \quad \text { for } v \in \Omega .
$$

(Recall that $F=\operatorname{grad} \log f, \Omega=f^{-1}\left(\boldsymbol{C}^{\times}\right)$and $O_{0}$ (resp. $O_{1}$ ) is the unique open (resp. closed) orbit in $\Omega$. The objects $F^{\vee}, \Omega^{\vee}, O_{0}^{\vee}$ and $O_{1}^{\vee}$ are defined in the same way starting from $f^{\vee}$ instead of $f$.)

Proof. (1) Since $O_{1}$ is Zariski closed in the affine variety $\Omega$, it is affine. Hence the isotropy group at a point of $O_{1}$ is reductive [20].

(2) By (1.15), (1.16) and (1.17), there exists a non-empty, $G$-stable open subset $U$ of $F(\Omega)$ such that, for any $x \in U, G \cdot x$ is closed in $F(\Omega)$. Since $F\left(O_{0}\right)$ is dense in $F(\Omega), U \cap F\left(O_{0}\right) \neq \phi$. Since $F\left(O_{0}\right)$ is a $G$-orbit, $F\left(O_{0}\right) \subset U$. Hence $F\left(O_{0}\right)=\overline{F\left(O_{0}\right)}=F(\Omega)$. By (1.15), $F\left(O_{0}\right)=F(\Omega)$ is a closed orbit in $\Omega^{\vee}$. Hence $F(\Omega)=O_{1}^{\vee}$.

(3) This follows from (2), (1.14) and (1.15). 
(4) Let $v^{\vee} \in O_{1}^{\vee}$. For any $v \in F^{-1}\left(v^{\vee}\right), F(v)=v^{\vee} \in O_{1}^{\vee}=F\left(O_{0}\right)$ by (2). Hence by $(1.10,(3)), v-F^{\vee} F(v) \in\left(T_{F(v)} F(\Omega)\right)^{\perp}$. Hence

$$
F^{-1}\left(v^{\vee}\right) \subset F^{\vee}\left(v^{\vee}\right)+\left(T_{v} \vee F(\Omega)\right)^{\perp} \text {. }
$$

Since $v^{\vee} \in O_{1}^{\vee}=F\left(O_{0}\right)$, we can write $v^{\vee}=F\left(v^{\prime}\right)$ with $v^{\prime} \in O_{0}$. By (1.13),

$$
F^{-1}\left(v^{\vee}\right)=F^{-1} F\left(v^{\prime}\right) \supset v^{\prime}+\left(T_{v^{\vee}} F(\Omega)\right)^{\perp} \text {. }
$$

Since $F^{\vee}\left(v^{\vee}\right)+\left(T_{v} \vee F(\Omega)\right)^{\perp}$ and $v^{\prime}+\left(T_{v} \vee F(\Omega)\right)^{\perp}$ are affine spaces of the same dimension, it follows from (2), (1.18.1) and (1.18.2) that

$$
F^{-1}\left(v^{\vee}\right)=F^{\vee}\left(v^{\vee}\right)+\left(T_{v} \vee F(\Omega)\right)^{\perp}=F^{\vee}\left(v^{\vee}\right)+\left(T_{v} \vee O_{1}^{\vee}\right)^{\perp} .
$$

Then, $\Phi$ defines an isomorphism $\pi^{-1}\left(v^{\vee}\right) \rightarrow F^{-1}\left(v^{\vee}\right)$ and $\Psi$ defines its inverse. In fact, if $\left(v, v^{\vee}\right) \in \pi^{-1}\left(v^{\vee}\right)$,

$$
\Phi\left(v, v^{\vee}\right)=v+F^{\vee}\left(v^{\vee}\right) \in\left(T_{v} \vee O_{1}^{\vee}\right)^{\perp}+F^{\vee}\left(v^{\vee}\right)=F^{-1}\left(v^{\vee}\right)
$$

by (1.18.3). Hence $F \Phi\left(v, v^{\vee}\right)=v^{\vee}$ and $\Psi \Phi\left(v, v^{\vee}\right)=\left(\Phi\left(v, v^{\vee}\right)-F^{\vee}\left(v^{\vee}\right), v^{\vee}\right)=$ $\left(v, v^{v}\right)$. On the other hand, it is easy to see that $\Phi \Psi(v)=v$. Thus we get the assertion.

Example 1.19. Let $V=V^{\vee}=\mathbb{C}^{2},\left\langle(x, y) \mid\left(x^{\prime}, y^{\prime}\right)\right\rangle=x x^{\prime}+y y^{\prime}$ and $G=\left(\mathbb{C}^{\times}\right)^{2}$. Define a $G$-action on $V$ by $\left(g_{1}, g_{2}\right)(x, y)=\left(g_{1} x, g_{2} y\right)$. Let $f(x, y)=x^{a} y^{b}$. For any $a$ and $b, O_{0}=O_{0}^{\vee}=C^{\times} \times \mathbb{C}^{\times}$.

(1) If $a=b=0$, then $\Omega=\mathbb{C}^{2}, O_{1}=\{(0,0)\}, O_{1}^{\vee}=\{(0,0)\}$ and $F^{-1}(0,0)=\mathbb{C}^{2}$.

(2) If $a=0, b>0$, then $\Omega=\mathbb{C} \times \mathbb{C}^{\times}, O_{1}=\{0\} \times \mathbb{C}^{\times}, O_{1}^{\vee}=\{0\} \times \mathbb{C}^{\times}$and $F^{-1}(0, y)=\mathbb{C} \times\{b / y\}$.

(3) If $a>0, b>0$, then $\Omega=\mathbb{C}^{\times} \times \mathbb{C}^{\times}, O_{1}=C^{\times} \times \mathbb{C}^{\times}, O_{1}^{\vee}=\mathbb{C}^{\times} \times \mathbb{C}^{\times}$and $F^{-1}(x, y)=\{(a / x, b / y)\}$.

Example 1.20. Let $V=V^{\vee}=M_{2 m, 3}(C),\left\langle v \mid v^{\vee}\right\rangle=\operatorname{Tr}\left(v^{*} \cdot v^{\vee}\right)$ and $G=C^{\times} \times$ $S p_{2 m}(\mathbb{C}) \times S O_{3}(\mathbb{C})$. Here $S p_{2 m}(\mathbb{C})=\left\{g \in G L_{2 m}(\mathbb{C}) \mid g^{*} J g=J\right\}$ with

$$
J=\left(\begin{array}{cc}
0 & 1_{m} \\
-1_{m} & 0
\end{array}\right),
$$

and $S_{3}(\boldsymbol{C})=\left\{g \in S L_{3}(\mathbb{C}) \mid g^{*} g=1\right\}$. (* means the transposition.) Define $G$ actions on $V$ and $V^{\vee}$ by $\left(t, g_{1}, g_{2}\right) v=t\left(g_{1} v g_{2}^{*}\right)$ and $\left(t, g_{1}, g_{2}\right) v^{\vee}=t^{-1}\left(g_{1}^{*-1} v^{\vee} g_{2}^{-1}\right)$ for $t \in \mathbb{C}^{\times}, g_{1} \in S p_{2 m}(\boldsymbol{C}), g_{2} \in S O_{3}(\boldsymbol{C}), v \in V$ and $v^{\vee} \in V^{\vee}$. Let $f(v)=f^{\vee}(v)=$ $-\operatorname{Tr}\left(\left(v^{*} J v\right)^{2}\right) / 2$ and $\phi\left(t, g_{1}, g_{2}\right)=t^{4}$. Then for $g=\left(t, g_{1}, g_{2}\right), f(g v)=\phi(g) f(v)$ and $f^{\vee}\left(g v^{\vee}\right)=\phi(g)^{-1} f^{\vee}\left(v^{\vee}\right)$. Let $v^{i}(1 \leq i \leq 3)$ be the column vectors of $v \in M_{2 m, 3}(\mathbb{C})$ and $\left(v^{i} v^{j}\right)=\left(v^{i}\right)^{*} J v^{j}$. Then $f(v)=\left(v^{1} v^{2}\right)^{2}+\left(v^{2} v^{3}\right)^{2}+\left(v^{3} v^{1}\right)^{2}$. Let 


$$
v_{0}=\left(\begin{array}{llllllllll}
1 & 0 & 0 & \cdots & 0 & & 0 & 0 & \cdots & 0 \\
0 & 1 & 0 & \cdots & 0 & & 0 & 0 & \cdots & 0 \\
0 & 0 & 0 & \cdots & 0 & & 1 & 0 & \cdots & 0
\end{array}\right)^{*}
$$

and

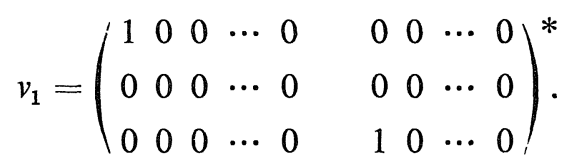

Then $\Omega=f^{-1}\left(C^{\times}\right)$consists of two orbits $O_{0}=G v_{0}$ and $O_{1}=G v_{1}$, where $O_{0}$ is open in $\Omega$ and $O_{1}$ is closed. The morphism $F=\operatorname{grad} \log f$ is given by

$$
F(v)=-2 J v v^{*} J v / f(v) .
$$

Hence

$$
F^{\vee} F(v)=-v\left(v^{*} J v\right)^{2} / f(v)
$$

and

$$
v-F^{\vee} F(v)=\frac{v}{f(v)}\left(\left(v^{2} v^{3}\right),\left(v^{3} v^{1}\right),\left(v^{1} v^{2}\right)\right)^{*} \cdot\left(\left(v^{2} v^{3}\right),\left(v^{3} v^{1}\right),\left(v^{1} v^{2}\right)\right) .
$$

The fibre of $F$ containing $v_{1}$ is

$$
\left\{\left(\begin{array}{cccccccc}
1 & 0 & \cdots & 0 & 0 & 0 & \cdots & 0 \\
0 & x_{2} & \cdots & x_{m} & 0 & x_{m+2} & \cdots & x_{2 m} \\
0 & 0 & \cdots & 0 & 1 & 0 & \cdots & 0
\end{array}\right)^{*}\right\}
$$

This example will be taken up again in (3.24).

Remark 1.21. Although the open orbit $O_{0}$ is uniquely determined by the prehomogeneous vector space $(G, \rho, V), \Omega=\Omega(f)$ and $O_{1}=O_{1}(f)$ depend on the choice of a relatively invariant polynomial $f$, as we have seen in (1.19). Moreover, we can show the following fact. Let $f_{1}$ and $f_{2}$ be relatively invariant polynomial functions on $V$. Then $O_{1}\left(f_{1}\right)=O_{1}\left(f_{2}\right)$ if and only if $\Omega\left(f_{1}\right)=\Omega\left(f_{2}\right)$, i.e., $f_{1}^{-1}(0)=f_{2}^{-1}(0)$.

Proof. Since $O_{1}\left(f_{i}\right)$ is the unique closed $G$-orbit in $\Omega\left(f_{i}\right), \Omega\left(f_{1}\right)=\Omega\left(f_{2}\right)$ implies $O_{1}\left(f_{1}\right)=O_{1}\left(f_{2}\right)$. Assume that $\Omega\left(f_{1}\right) \neq \Omega\left(f_{2}\right)$, i.e., $f_{1}^{-1}(0) \neq f_{2}^{-1}(0)$. Let $\phi_{i}$ be the character of $f_{i}$, and $f_{i}^{\vee}$ a relatively invariant polynomial on $V^{\vee}$ of character $\phi_{i}^{-1}$ (cf. (1.5)). As is seen from the proof of (1.5), $f_{i}^{\vee}$ can be obtained from $f_{i}$ by the complex conjugation of the coefficients of $f_{i}$ (up to scalar multiple). Hence $f_{1}^{\vee-1}(0) \neq f_{2}^{\vee-1}(0)$. Assume that $f_{1}^{\vee-1}(0) \mp f_{2}^{\vee-1}(0)$. Then for any natural number $s, f_{2}^{\vee s} / f_{1}^{\vee}$ is not a polynomial. Since $f_{1}$ (grad) $f_{2}^{\vee s}$ and $f_{2}^{\vee s} / f_{1}^{\vee}$ 
correspond to the same character $\phi_{1} \phi_{2}^{-s}$ and $f_{1}(\operatorname{grad}) f_{2}^{\mathrm{v} s}$ is a polynomial, $f_{1}(\operatorname{grad}) f_{2}^{\vee_{s}}=0$. Hence we can show, as in the proof of $(1.8)$, that

$$
f_{1}\left(\operatorname{grad} \log f_{2}^{\vee}\right) \equiv 0 \text {. }
$$

On the other hand, by (1.7) and (1.8),

$$
f_{1}\left(\operatorname{grad} \log f_{1}^{\vee}\right) \neq 0 \text {. }
$$

Since $\left(\operatorname{grad} \log f_{i}^{\vee}\right)\left(O_{0}^{\vee}\right)=O_{1}\left(f_{i}\right)$ by $(1.18,(2)), O_{1}\left(f_{1}\right) \neq O_{1}\left(f_{2}\right)$.

\section{§2. $\mathbb{D}$-modules}

2.0. In this section, we review the theory of systems of differential equations.

\subsection{Differential operators}

\subsubsection{Weyl algebra}

For an affine variety $V$ over $\mathbb{C}$, we denote the ring of regular functions on $V$ by $A(V)$ or $\mathbb{C}[V]$.

Let $V=\mathbb{C}^{n}$ and $\left\{x_{1}, \cdots, x_{n}\right\}$ be its coordinate system. The Weyl algebra $D(V)$ is the $\mathbb{C}$-algebra defined by the following presentation:

(1) $D(V)$ is generated by $2 n$ elements $x_{1}, \cdots, x_{n}, \frac{\partial}{\partial x_{1}}, \cdots, \frac{\partial}{\partial x_{n}}$.

(2) These generators satisfy the commutation relations $\left[x_{i}, x_{j}\right]=0$, $\left[\frac{\partial}{\partial x_{i}}, \frac{\partial}{\partial x_{j}}\right]=0$, and $\left[\frac{\partial}{\partial x_{i}}, x_{j}\right]=\delta_{i j}$, where $[P, Q]=P Q-Q P$ and $\delta_{i j}$ is the Kronecker delta.

For an affine open subset $U$ of $V$, let $D(U)=A(U) \otimes_{A(V)} D(V)$. Sometimes we write $\partial_{i}$ for $\frac{\partial}{\partial x_{i}}, A$ for $A(U)$, and $D$ for $D(U)$. We call an element $P$ of $D$ a differential operator and define its $\operatorname{order}$ ord $(P)$ in the usual way. Let $D_{k}=$ $\{P \in D \mid \operatorname{ord}(P) \leq k\}$. Then $\left\{D_{k}\right\}$ gives a filtered ring structure in $D$. Let $\operatorname{gr}_{k}(D)$ $=D_{k} / D_{k-1}$ and $\operatorname{gr}(D)=\oplus_{k \in Z} \operatorname{gr}_{k}(D)$. The $\operatorname{gr}(D(U))$ has a natural ring structure and is isomorphic to $\mathbb{C}\left[U \times V^{\vee}\right]$ as a graded ring, where $V^{\vee}$ is the dual space of $V$. Let $\sigma=\sigma_{k}: D_{k} \rightarrow D_{k} / D_{k-1}$ be the natural projection. For a differential operator $P$ of order $k$, we call $\sigma_{k}(P)$ the principal symbol of $P$. For a left ideal $L$ of $D(U)$, let

$$
\operatorname{gr}(L)=\bigoplus_{k \in Z} \sigma_{k}\left(L \cap D_{k}\right)
$$


Then $\operatorname{gr}(L)$ is an ideal of $\operatorname{gr}(D(U))=C\left[U \times V^{\vee}\right]$.

2.1.2. Define an anti-automorphism * of $D$ by $\partial_{i}^{*}=-\partial_{i}$ and $a^{*}=a(a \in A)$. For $P \in D$, we call $P^{*}$ the adjoint operator of $P$. Let $\Omega$ be the $A$-module $A \cdot d x_{1}$ $\wedge \cdots \wedge d x_{n}$. For a right $D$-module $M, \operatorname{Hom}_{A}(\Omega, M)=M \otimes_{A} \Omega^{-1}$ has a left $D$-module structure defined by $P\left(m \otimes\left(d x_{1} \wedge \cdots \wedge d x_{n}\right)^{-1}\right)=m P^{*} \otimes\left(d x_{1} \wedge \cdots \wedge\right.$ $\left.d x_{n}\right)^{-1}$. (As far as we deal with $D$-modules over an open set of $C^{n}, \otimes_{A} \Omega^{-1}$ is not important. In such a case, we omit to write it if the notation becomes too complicated.)

2.1.3. There are several kinds of 'differential operators'. Let us consider relations among them.

For a point $v \in V$, let $A_{v}$ be the localization of $A$ at $v$, and $m_{v}$ its maximal ideal. Let $\left(\hat{A_{v}}, \hat{m}_{v}\right)$ be the $m_{v}$-adic completion of $\left(A_{v}, m_{v}\right)$. Let $\tilde{A_{v}}$ be the ring of germs of holomorphic functions at $v$, and $\widetilde{m}_{v}$ its maximal ideal. Then the $\tilde{m}_{v}$-adic completion of $\left(\tilde{A_{v}}, \widetilde{m}_{v}\right)$ is also $\left(\hat{A}_{v}, \hat{m}_{v}\right)$. Since $A_{v}$ is noetherian, $\hat{A}_{v}$ is faithfully flat over $A_{v}\left[3\right.$; Chap.3, §3, Prop.9]. Similarly, $\hat{A}_{v}$ is faithfully flat over $\tilde{A_{v}}$. Hence $\tilde{A_{v}}$ is faithfully flat over $A_{v}$ (cf. [3; Chap. 1, $\S 3$, no. 4, Remark (2)]). Hence any $A_{v}$-module $M$ can be regarded as a submodule of $\tilde{A_{v}} \otimes_{A_{v}} M[3$; Chap. 1, §3, Prop.9]. Since $A_{v}$ is flat over $A$ [3; Chap.2, §2, Theorem 1], $\widetilde{A_{v}}$ is flat over $A$. Let $D_{v}=A_{v} \otimes_{A} D$ and $\tilde{D_{v}}=\tilde{A_{v}} \otimes_{A} D$. Since $\tilde{A_{v}}$ is flat over $A, \tilde{D}_{v}$ is flat over $D$. For a point $p=\left(v, v^{\vee}\right)$ of $V \times V^{\vee}$, let $E_{p}$ be the ring of germs of microdifferential operators (of finite order) at $p$. (See $[15 ; 2.2 .2]$ for the definition. Here we consider $V \times V^{\vee}$ as the cotangent bundle of $V$.) Since $E_{p}$ is flat over $\widetilde{D}_{v}$ [26; Chap. 2, 3.4, Remark 2], $E_{p}$ is flat over $D$.

2.1.4. Let $V=V^{\prime} \oplus V^{\prime \prime},\left\{x_{1}^{\prime}, \cdots, x_{a}^{\prime}\right\}$ and $\left\{x_{1}^{\prime \prime}, \cdots, x_{b}^{\prime \prime}\right\}$ be coordinate systems of $V^{\prime}$ and $V^{\prime \prime}$, and $I$ (resp. $I^{\prime \prime}$ ) the ideal of $A(V)$ (resp. $A\left(V^{\prime \prime}\right)$ ) generated by $x_{1}^{\prime \prime}, \cdots, x_{b}^{\prime \prime}$. Let $i: V^{\prime} \rightarrow V$ be the inclusion mapping and

$$
D_{V^{\prime} \rightarrow V}=(A(V) / I) \otimes_{A(V)} D(V)=D(V) / I D(V)=D\left(V^{\prime}\right) \otimes_{c}\left(D\left(V^{\prime \prime}\right) / I^{\prime \prime} D\left(V^{\prime \prime}\right)\right) .
$$

Then $D_{V^{\prime} \rightarrow V}$ is a left $D\left(V^{\prime}\right)$-module and a right $D(V)$-module. Let $v=v^{\prime} \oplus v^{\prime \prime}$, $D_{V^{\prime} \rightarrow V, v}=D_{V^{\prime} \rightarrow V} \otimes_{A(V)} A_{v}, \tilde{D}_{V^{\prime} \rightarrow V, v}=D_{V^{\prime} \rightarrow V} \otimes_{A_{v}} \tilde{A}_{v}, D_{v^{\prime}}=A_{v^{\prime}} \otimes_{A\left(V^{\prime}\right)} D\left(V^{\prime}\right)$ and $\tilde{D}_{v^{\prime}}$ $=\tilde{A_{v^{\prime}}} \otimes_{A_{v^{\prime}}} D_{v^{\prime}}$. Then $D_{V^{\prime} \rightarrow V, v}$ is a left $D_{v^{\prime}}$-module, and $\tilde{D}_{V^{\prime} \rightarrow V, v}$ is a left $\widetilde{D}_{v^{\prime}}$ module. Let $\tilde{A_{v}} D\left(V^{\prime}\right)$ be the subring of $\tilde{D}_{v}$ generated by $\tilde{A_{v}}$ and $D\left(V^{\prime}\right)$. Then $\tilde{D}_{v}$ is a free left $\tilde{A_{v}} D\left(V^{\prime}\right)$-module generated by $\left\{\left(\frac{\partial}{\partial x_{1}^{\prime \prime}}\right)^{j_{1}} \cdots\left(\frac{\partial}{\partial x_{b}^{\prime \prime}}\right)^{j_{b}} \mid j_{i} \geq 0\right\}$. Hence $\tilde{D}_{v}$ is a faithfully flat left $\tilde{A_{v}} D\left(V^{\prime}\right)$-module. Since $\tilde{A_{v}} D\left(V^{\prime}\right) / I \tilde{A_{v}} D\left(V^{\prime}\right)=$ $\widetilde{D}_{v^{\prime}}$ and $\widetilde{D}_{v} / I \widetilde{D}_{v}=\widetilde{D}_{V^{\prime} \rightarrow V, v}, \widetilde{D}_{V^{\prime} \rightarrow V, v}$ is a faithfully flat left $\widetilde{D}_{v^{\prime}}$-module. Let $1_{V^{\prime} \rightarrow V}=$ 
$\left(1_{D(V)} \bmod I D(V)\right)$. Since $I D(V)=\left\{P \in D(V) \mid P A_{v} \subset I A_{v}\left(P\right.\right.$ acts on $A_{v}$ as a differential operator)\}, there do not exist elements $P \in D(V)-I D(V)$ and $s \in A_{v}-$ $m_{v}$ such that $P_{s} \in I D(V)$ (multiplication of differential operators $P$ and $s$ ). Hence $D_{V^{\prime} \rightarrow V}$ may be regarded as a subset of $D_{V^{\prime} \rightarrow V, v}$. By (2.1.3), $D_{V^{\prime} \rightarrow V, v}$ may be regarded as a submodule of $\widetilde{D}_{V^{\prime} \rightarrow V, v}$. Hence $1_{V^{\prime} \rightarrow V}$ may be regarded as an element of $D_{V^{\prime} \rightarrow V, v}$ or $\widetilde{D}_{V^{\prime} \rightarrow V, v}$.

Lemma 2.1.5. Let $M$ be a right $\widetilde{D}_{v^{\prime}}$-module. For an element $u$ of $M$, the following conditions are equivalent:

(1) $u \otimes 1_{V^{\prime} \rightarrow V}=0$ in $M \otimes \tilde{D}_{v^{\prime}} \tilde{D}_{V^{\prime} \rightarrow V, v}$.

(2) The image of $u \widetilde{D}_{v^{\prime}} \otimes \widetilde{D}_{v^{\prime}} \widetilde{D}_{V^{\prime} \rightarrow V, v}$ in $M \otimes \widetilde{D}_{v^{\prime}} \tilde{D}_{V^{\prime} \rightarrow V, v}$ is zero.

(3) $u \tilde{D}_{v^{\prime}} \otimes \tilde{D}_{v^{\prime}}, \widetilde{D}_{V^{\prime} \rightarrow V, v}=0$.

(4) $u=0$.

Proof. Since $1_{V^{\prime} \rightarrow V}$ is a generator of the right $\widetilde{D}_{v}$-module $\widetilde{D}_{V^{\prime} \rightarrow V, v},(1) \Leftrightarrow(2)$. Since $\widetilde{D}_{V^{\prime} \rightarrow V, v}$ is a flat left $\widetilde{D}_{v^{\prime}}$-module, (2) $\Leftrightarrow(3)$. Since $\widetilde{D}_{V^{\prime} \rightarrow V, v}$ is faithfully flat left $\widetilde{D}_{v^{\prime}}$-module, (3) $\Leftrightarrow(4)$.

2.1.6. Let $M$ be a $D$-module generated by a single element $u$ and $N$ another $D$-module. Sometimes, we identify a $D$-module homomorphism $\varphi: M \rightarrow N$ with the element $\varphi(u)$ of $N$, and call $\varphi$ or $\varphi(u)$ a solution of $M$ (in $N$ ). Cf. $[15 ; 2.1]$.

2.1.7. Let $M$ be a $D$-module and $f \in A(U)$. Considering $M$ as an $A$ module, we can define its localization by $f^{-1}$, whch we shall denote by $M\left[f^{-1}\right]$. (An element of $M\left[f^{-1}\right]$ can be expressed as $f^{-l} u$ with $u \in M$ and an integer $l \geq 0$, and $f^{-l} u=0$ if and only if $f^{N} u=0$ for a sufficiently large $N$.) Then $M\left[f^{-1}\right]$ has a natural $D$-module structure such that

$$
\partial_{i}\left(f^{-l} u\right)=-l f^{-l-1} \frac{\partial f}{\partial x_{i}} u+f^{-l}\left(\partial_{i} u\right) .
$$

\subsection{Characteristic variety}

\subsubsection{Support}

Let $V$ be a smooth, irreducible, affine variety over $\mathbb{C}, A=A(V)$, and $M$ an $A$-module. For an element $u$ of $M$, let

$$
\operatorname{ann}_{A}(u)=\{a \in A \mid a u=0\}
$$

and

$$
\operatorname{supp}(u)=\left\{v \in V \mid a(v)=0 \quad \text { for any } a \in \operatorname{ann}_{A}(u)\right\} .
$$


Let $\operatorname{supp}(M)$ be the union of $\operatorname{supp}(u)$ for $u \in M$, and call it the support of $M$. If $M$ is finitely generated, $\operatorname{supp}(M)$ is Zariski closed in $V$.

2.2.2. Let $M$ be a finitely generated $A$-module, $C$ an irreducible algebraic set of $V, r=\operatorname{codim}_{V}(C), I=\{a \in A \mid a \equiv 0$ on $C\}$ and $S=A-I$. Assume that there is an affine open subset $W$ of $V$ such that $W \cap C \neq \phi$ and

(1) $W \cap \operatorname{supp}(M)=\phi$, or

(2) $W \cap \operatorname{supp}(M)=W \cap C$.

Then $S^{-1} A$ is a regular local ring of dimension $r, S^{-1} M$ is a finitely generated $S^{-1} A$-module, and is annihilated by some power of the maximal ideal $S^{-1} I$ of $S^{-1} A$. (In fact, $A(W) \otimes_{A} M$ is already annihilated by some power of $A(W) I$.) Hence length ${ }_{S^{-1} A}\left(S^{-1} M\right)$ is finite, which we shall denote by $m(C)=m(C, M)$ and call the multiplicity of $M$ along $C$. See [29] for regular local rings.

\subsubsection{Characteristic variety}

Let $V=C^{n}$ and $U$ be an affine open subset of $V$. Let $M$ be a (left) $D(U)$ module, and $F=\left(F_{k} M\right)_{k \in Z}$ an increasing filtration of $M$ by finitely generated $A(U)$-submodules such that

$$
\bigcup_{k \in Z} F_{k} M=M \text { and } D_{l}\left(F_{k} M\right) \subset F_{k+l} M .
$$

(Recall that $\left\{D_{l}\right\}$ is the order filtration of $D$.) Then $\operatorname{gr}(M)$ naturally becomes a $\operatorname{gr}(D)$-module, where $\operatorname{gr}_{k}(M)=F_{k} M / F_{k-1} M$ and $\operatorname{gr}(M)=\bigoplus_{k \in Z} \operatorname{gr}_{k}(M)$. Such a filtration $F$ is said to be $\operatorname{good}$ if $\operatorname{gr}(M)$ is finitely generated as a $\operatorname{gr}(D)$-module. A $D$-module $M$ has a good filtration if and only if $M$ is finitely generated as a $D$-module.

If $F$ is a good filtration of a $D(U)$-module $M$, the support of $\operatorname{gr}(D)$-module $\operatorname{gr}(M)$ is an algebraic subset of $U \times V^{\vee}$, which is denoted by $\operatorname{ch}(M)$ and called the characteristic variety. As is easily seen, $\operatorname{ch}(M)$ does not depend on the choice of a good filtration. A finitely generated $D$-module $M$ is called holonomic if $\operatorname{dim} \operatorname{ch}(M) \leq n$.

\subsubsection{Characteristic cycle}

Let $C$ be an irreducible algebraic subset of $U \times V^{\vee}$. Assume that there exists an affine open set $W$ of $U \times V^{\vee}$ such that $W \cap C \neq \phi$ and

(1) $W \cap \operatorname{supp}(M)=\phi$, or

(2) $W \cap \operatorname{supp}(M)=W \cap C$.

Then we can consider the multiplicity of $\operatorname{gr}(M)$ along $C$, which we shall call the multiplicity of $M$ along $C$ and denote by $m(C)=m(C, M)$.

Let $i(M)$ be the set of irreducible components of $\operatorname{ch}(M)$ and consider the 
formal sum

$$
\operatorname{chl}(M)=\sum_{\sigma \in i(M)} m(C, M) C,
$$

which we call the characteristic cycle of $M$. The characteristic cycle is also independent of the choice of a good filtration.

Lemma 2.2.5. For a finitely generated $D$-module $M$ and a point $p=\left(v, v^{v}\right)$ $\in V \times V^{\vee}$, the following conditions are equivalent to each other:

(1) $p \in \operatorname{ch}(M)$.

(2) For some $u \in M, p \in \operatorname{ch}(D u)$.

(3) For some $u \in M, E_{p} \otimes_{D}(D u) \neq 0$.

(4) $E_{p} \otimes_{D} M \neq 0$.

Proof. (1) $\Leftrightarrow(2)$ is obvious. Since $E_{p}$ is flat over $D$ (cf. (2.1.3)), $E_{p} \otimes_{D}(D u)$ can be regarded as an $E_{p}$-submodule of $E_{p} \otimes_{D} M$ for any $u$. Hence (3) $\Leftrightarrow$ (4). For a given element $u \in M$, let $I=\operatorname{ann}_{D}(u)$. In order to prove (2) $\Leftrightarrow(3)$, it is enough to prove the equivalence of the following conditions:

(a) $p \in \operatorname{ch}(D u)$.

(b) $p$ is a common zero of the ideal $\operatorname{gr}(I)$ of $\operatorname{gr}(D)=\mathbb{C}\left[V \times V^{\vee}\right]$.

(c) $p$ is a common zero of the ideal $\operatorname{gr}\left(\tilde{A_{v}} \otimes I\right)$ of $\operatorname{gr}\left(\tilde{D}_{v}\right)=\tilde{A}_{v} \otimes_{C} \mathbb{C}\left[V^{\vee}\right]$.

(d) $E_{p} \otimes_{D}(D u) \neq 0$.

(In (c), the filtration of $\widetilde{D}_{v}$ is given by $\tilde{D}_{k, v}=\tilde{A}_{v} \otimes_{A} D_{k}$ and $\operatorname{gr}(-)$ is defined as before.) (a) $\Leftrightarrow$ (b) is obvious. Since $\tilde{A_{v}}$ is flat over $A$, it follows that $\tilde{A_{v}} \otimes_{A}(D u)$ $=\tilde{D}_{v} /\left(\tilde{A}_{v} \otimes I\right), \tilde{D}_{k, v} \cap\left(\tilde{A_{v}} \otimes I\right)=\tilde{A}_{v} \otimes\left(D_{k} \cap I\right)$ and $\operatorname{gr}\left(\tilde{A_{v}} \otimes I\right)=\tilde{A}_{v} \otimes \operatorname{gr}(I)[3 ;$ Chap.1 $\S 2$, Prop.6]. Hence $(\mathrm{b}) \Leftrightarrow(\mathrm{c})$. (c) $\Leftrightarrow(\mathrm{d})$ is proved in $[15 ; 2.3 .5]$.

Lemma 2020\% Let

$$
0 \rightarrow M^{\prime} \rightarrow M \rightarrow M^{\prime \prime} \rightarrow 0
$$

be an exact sequence of $D$-modules. If $M$ is finitely generated, then $M^{\prime}$ and $M^{\prime \prime}$ are also finitely generated, and

$$
m(\mathbb{C}, M)=m\left(\mathbb{C}, M^{\prime}\right)+m\left(\mathbb{C}, M^{\prime \prime}\right)
$$

for any irreducible component $\mathbb{C}$ of $M$.

Proof. Consider a good filtration of $M$ and filtrations of $M^{\prime}$ and $M^{\prime \prime}$ induced from it. Then

$$
0 \rightarrow \operatorname{gr}\left(M^{\prime}\right) \rightarrow \operatorname{gr}(M) \rightarrow \operatorname{gr}\left(M^{\prime \prime}\right) \rightarrow 0
$$


is exact. By the exactness of the localization as a functor, and by the additivity of the length, we get the assertion.

Lemma 2.2.7. For $a \quad D(U)$-module $M$ and $u \in M, \quad \operatorname{supp}(u)=\operatorname{ch}(D u) \cap$ $(U \times\{0\})$.

The assertion follows from the equivalence $(a) \Leftrightarrow(b)$ in the proof of $(2.2 .5)$.

2.3. In this paragraph, we study $D$-modules related to the complex power $f^{s}$ of a polynomial function $f(x)$. A main purpose of this paragraph is to give (2.3.11).

2.3.H. Let $V=\mathbb{C}^{n}, f$ be a polynomial function on $V, B$ a connected, simply connected open subset of $\Omega=f^{-1}\left(C^{\times}\right), f^{s}$ a single-valued branch of $f(x)^{s}((s, x)$ $\in C \times B), D[s]=D \otimes_{C} C[s]$ and $D[s] f^{s}$ the $D[s]$-module generated by $f^{s}$. For a given complex number $\alpha$, let $f^{\alpha}=\left(f^{s} \bmod (s-\alpha) D[s] f^{s}\right)$. Then $f^{\alpha}$ is a generator of the $D$-module $D[s] f^{s} /(s-\alpha) D[s] f^{s}$. Note that $f^{\alpha}$ is only an element of an abstract $D$-module $D f^{\alpha}$, and not a function.

2.3.2 ([14], [16]). Let $M$ be a $D$-module and $u$ an element of $M$. Consider the left ideal $I$ of $D[s]$ consisting of differential operators $P(s) \in D[s]$ such that

$$
\left(f^{m-s} P(s) f^{s}\right) u=0
$$

holds in $\boldsymbol{C}[s] \otimes_{\boldsymbol{C}} M$ for a sufficiently large integer $m$. Note that $f^{m-s} P(s) f^{s} \in$ $D[s]$ if $m$ is large enough. Let $N=D[s] / I$ and denote the element $(1 \bmod I)$ by $f^{s} u$. Then $N=D[s]\left(f^{s} u\right)$. By the definition of $N$,

$$
\begin{gathered}
f^{m} P(s)\left(f^{s} u\right)=0 \text {, if and only if } P(s)\left(f^{s} u\right)=0, \text { and } \\
P(s)\left(f^{s} u\right)=0 \text {, if and only if } P(s+m)\left(f^{m} \cdot f^{s} u\right)=0,
\end{gathered}
$$

for $P(s) \in D[s]$ and $m \geq 0$. For a complex number $\alpha$, let $N(\alpha)=N /(s-\alpha) N$, and $f^{\alpha} u=\left(f^{s} u \bmod (s-\alpha) N\right)$. Then $N(\alpha)=D\left(f^{\alpha} u\right)$.

Note that if $M=A(V)$ and $u=1$, the $D[s]$-module $D[s]\left(f^{s} u\right)$ is naturally isomorphic to the $D[s]$-module $D[s] f^{s}$ given in (2.3.1).

2.3.5. Let $C[s, t]$ be the $C$-algebra defined by the relation $t s=(s+1) t$, and $D[s, t]=D \otimes_{C} C[s, t]$. Then $D[s]\left(f^{s} u\right)$ has a $D[s, t]$-module structure defined by

$$
t\left(P(s) f^{s} u\right)=P(s+1) f \circ f^{s} u
$$


This definition is well-defined by (2.3.4).

2.3.6. For a polynomial $c(s) \in \mathbb{C}[s]$, let

$$
\begin{array}{ll}
A_{+}=A_{+}(c)=\{\alpha \in \mathbb{C} \mid c(\alpha+j) \neq 0 & \text { for } j=0,1,2, \cdots\}, \\
A_{-}=A_{-}(c)=\{\alpha \in \mathbb{C} \mid c(\alpha-j) \neq 0 & \text { for } j=1,2, \cdots\}
\end{array}
$$

Henceforth, we assume that there exist a polynomial $c(s) \in \mathbb{C}[s]$ and a differential operator $P(s) \in D[s]$ such that

$$
P(s)\left(f \circ f^{s} u\right)=c(s) f^{s} u \text {. }
$$

(It is known [14] that such $c(s)$ and $P(s)$ exist if $M$ is holonomic.)

Lemma 2.3.8 ([16; Lemma 2.3]). For $\alpha \in A_{-}(c), D\left(f^{\alpha} u\right)$ is naturally isomorphic to $D\left(f^{\infty} u\right)\left[f^{-1}\right]$ with the natural D-module structure.

(See (2.1.7) for $(-)\left[f^{-1}\right]$. Although we do not assume $M$ to be holonomic, the proof of [16; Lemma 2.3] works if we replace 'bijective' in the line 14 of page 175 with 'injective'.)

Lemma 2.3.9. If $c(\alpha-1) \cdots c(\alpha-l) \neq 0$, then $f^{\alpha} u \rightarrow f^{l} \cdot f^{\alpha-l} u$ gives an isomorphism $D\left(f^{\alpha} u\right) \rightarrow D\left(f^{\alpha-l} u\right)$. Especially, if $\alpha \in A_{-}(c)$, the assumption is satisfied.

Proof. The inverse morphism is given by

$$
f^{\alpha-l} u \rightarrow(c(\alpha-l) \cdots c(\alpha-1))^{-1}(P(\alpha-l) \cdots P(\alpha-1))\left(f^{\alpha} u\right) .
$$

Lemma 2.3.10. Assume that $f$ is not a zero divisor of $D u(\subset M)$. Then $D u$ is a quotient of $N(0)=D\left(f^{0} u\right)$.

Proof. Define the $D$-module homomorphism $D[s] f^{s} u \rightarrow D u$ by

$$
Q(s)\left(f^{s} u\right) \rightarrow Q(0) u .
$$

If $Q(s) f^{s} u=0$, then $\left(f^{m-s} Q(s) f^{s}\right) u=0$ for a sufficiently large integer $m$. Let $f^{m-s} Q(s) f^{s}=\sum s^{j} Q_{j}$. Then $Q_{j} u=0$. Since $Q_{0}=f^{m} Q(0), f^{m} Q(0) u=0$. But we are assuming that $f$ is not a zero divisor of $D u$. Hence $Q(0) u=0$, and, consequently, the above morphism is well-defined. Obviously, it is surjective and its kernel contains $s D[s]\left(f^{s} u\right)$. Thus we get a surjective homomorphism

$$
D[s]\left(f^{s} u\right) / s D[s]\left(f^{s} u\right) \rightarrow D u .
$$

Lemma 2.3.11. Assume that $f$ is not a zero divisor of $D u$. If $0 \in A_{-}(c)$, then $D u=(D u)\left[f^{-1}\right]$. 
(See (2.3.6) for $A_{-}$, and (2.3.7) for $c$.)

Proof. By (2.3.8), $N(0)=D\left(f^{0} u\right)$ is isomorphic to $D\left(f^{0} u\right)\left[f^{-1}\right]$. Hence, by (2.3.10), we get a surjective homomorphism $\varphi:\left(D f^{0} u\right)\left[f^{-1}\right] \rightarrow D u$. It is enough to prove that $\operatorname{ker}(\varphi)=0$.

If $\varphi\left(Q f^{0} u\right)=0$ with $Q \in D$, then $Q u=0$. If $m$ is a sufficiently large integer, then, as elements of $D[s], f^{m-s} Q f^{s}=f^{m} Q+s R(s)$ with some $R(s) \in D[s]$. Hence $\left(f^{m-s} Q f^{s}\right) u=s R(s) u$. For a sufficiently large integer $l$, there exists $T(s) \in D[s]$ such that $f^{s-m} R(s)=T(s) f^{s-l}$. Then as elements of $D[s], f^{m-s} Q f^{s}$ $=f^{m} Q+s f^{m-s} T(s) f^{s-l}$, and $f^{m-s}\left(Q f^{l}-(s+l) T(s+l)\right) f^{s}=f^{m+l} Q$. Hence $\left(Q f^{l}-(s+l) T(s+l)\right) f^{s} u=0$ in $D[s]\left(f^{s} u\right)$, and $\left(Q f^{l}\right) \cdot\left(f^{-l} u\right)=0$ in $D\left(f^{-l} u\right)$. Since 0 belongs to $A_{-}, D\left(f^{0} u\right) \rightarrow D\left(f^{-l} u\right)\left(f^{0} u \rightarrow f^{l} \cdot f^{-l} u\right)$ is an isomorphism by (2.3.9). Hence $Q f^{0} u=0$.

In general, if $\varphi\left(f^{-m} \cdot Q f^{0} u\right)=0$, then $\varphi\left(Q f^{0} u\right)=0, Q f^{0} u=0$, and $f^{-m}$. $Q f^{0} u=0$. Hence $\operatorname{ker}(\varphi)=0$.

2.4. In this paragraph, we recall known facts about the characteristic varieties of $D[s] f^{s}$ and $D f^{\alpha}$. We include (2.4.3) and (2.4.4) here in order to make [13] accessible.

2.4.1. Let $V^{\vee}=\left\{y=\left(y_{1}, \cdots, y_{n}\right)\right\}$ be the dual space of $V=\boldsymbol{C}^{n}=$ $\left\{\left(x_{1}, \cdots, x_{n}\right)\right\}, p: \boldsymbol{C} \times V \times V^{\vee} \rightarrow V \times V^{\vee}$ the natural projection, $\Omega=f^{-l} \boldsymbol{C}^{\times}$,

$$
\begin{aligned}
& \tilde{W}^{\prime}=\left\{(s, x, s \text { grad } \log f(x)) \in C^{\times} \times \Omega \times V^{\vee}\right\}, \\
& \tilde{W}=\text { the Zariski closure of } \tilde{W}^{\prime} \text { in } C \times V \times V^{\vee}, \\
& \tilde{W}_{0}=\left(\{0\} \times V \times V^{\vee}\right) \cap \tilde{W}, \\
& W^{\prime}=p\left(\tilde{W}^{\prime}\right), \\
& W=\text { the Zariski closure of } W^{\prime} \text { in } V \times V^{\vee}, \text { and } \\
& W_{0}=\left\{(x, y) \in W \mid f(x) y_{1}=\cdots=f(x) y_{n}=0\right\} .
\end{aligned}
$$

2.4.2. Since $\tilde{W}^{\prime}$ is Zariski closed in $C^{\times} \times \Omega \times V^{\vee}$,

$$
\tilde{W}^{\prime}=\tilde{W} \cap\left(C^{\times} \times \Omega \times V^{\vee}\right)=\tilde{W}-\{s f(x)=0\} .
$$

In an irreducible variety over $\boldsymbol{C}$, a Zariski open set is everywhere dense with respect to the classical topology. Hence the closure of $\tilde{W}^{\prime}$ with respect to the classical topology coincides with $\tilde{W}$. Similarly, the closure of $W^{\prime}$ with respect to the classical topology coincides with $W$.

Lemma 2.4.3. ([13; Lemma 5.5]). If $\left(x_{0}, y_{0}\right) \in W$ and $f\left(x_{0}\right)=0$, then there exists a sequence $\left(s_{k}, x_{k}, y_{k}\right) \in C^{\times} \times W^{\prime}$ such that $y_{k}=s_{k}(\operatorname{grad} \log f)\left(x_{k}\right), s_{k} \rightarrow 0$ 
and $\left(x_{k}, y_{k}\right) \rightarrow\left(x_{0}, y_{0}\right)$.

Sublemma. Let $W$ be an algebraic variety over an algebraically closed field $k, W^{\prime}$ an open dense subset of $W$, and $p$ a point of $W$. Then there exists an irreducible curve $C \subset W$ such that $p \in C \oplus W \backslash W^{\prime}$.

Proof of (2.4.3). Let $C \subset W$ be an irreducible algebraic curve such that $p:=\left(x_{0}, y_{0}\right) \in C \mp W \backslash W^{\prime}, a: C^{\prime} \rightarrow C$ the normalization of $C, p^{\prime}$ a point of $C^{\prime}$ lying over $p$, and $b: C^{\prime} \rightarrow \mathbb{P}^{1}$ any branched covering. Put $D=\{t \in \mathbb{C}|| t \mid<1\}$ and $d(x)=x^{e}(x \in D)$ for a given natural number $e$. If the ramification index of $b$ at $p^{\prime}$ is $e$, then the branched covering $b: C^{\prime} \rightarrow \mathbb{P}^{1}$ is analytically isomorphic to $d: D \rightarrow D$ in a neighbourhood of $p^{\prime}$. As the composition of the local isomorphism $(D, 0) \rightarrow\left(C^{\prime}, p^{\prime}\right)$ and the natural projection $\left(C^{\prime}, p^{\prime}\right) \rightarrow(C, p)$, we get a holomorphic curve $c: D \rightarrow C(\subset W)$ such that $c(0)=p$ and $c(t) \in W^{\prime}$ for $0<|t| \ll$ 1. By a change of variable $t$, we may assume that $c(t)$ is holomorphic and lying in $W^{\prime}$ for $0<|t| \leq 2$. Let $c(t)=(x(t), y(t))$. For $t_{0} \in D \backslash\{0\}$, let

$$
\overline{x_{i}}\left(t_{0}\right)=\max _{|t| \leq 2\left|t_{0}\right|}\left|x_{i}(t)\right| \text {. }
$$

Then $\lim _{t \rightarrow 0} \bar{x}_{i}(t)=0$ and

$$
\left|t_{0}\right| \circ\left|\dot{x}_{i}\left(t_{0}\right)\right| \leq \max _{\left|t-t_{0}\right| \leq\left|t_{0}\right|}\left|x_{i}(t)\right| \leq \overline{x_{i}}\left(t_{0}\right)
$$

by the Cauchy's inequality concerning analytic functions of one variable. Let $f(x(t))=\sum_{i \geq 0} f_{i} t^{i}$. If $f_{0}=\cdots=f_{m-1}=0$ and $f_{m} \neq 0$, then $m>0$,

$$
f(x(t))=f_{m} t^{m}+O\left(t^{m+1}\right), \text { and } 2 t \frac{d}{d t} f(x(t))=2 m f_{m} t^{m}+O\left(t^{m+1}\right) .
$$

Hence

$$
\begin{gathered}
|f(x(t))| \leq\left|2 t \frac{d}{d t} f(x(t))\right| \leq 2 \sum_{i=1}^{n}|t| \cdot\left|\dot{x}_{\dot{i}}(t)\right| \cdot\left|\frac{\partial f}{\partial x_{i}}(x(t))\right| \\
\leq 2 \sum_{i=1}^{n} \overline{x_{i}}(t) \cdot\left|\frac{\partial f}{\partial x_{i}}(x(t))\right| \leq 2|\bar{x}(t)| \cdot|(\operatorname{grad} f)(x(t))|
\end{gathered}
$$

for $|t| \ll 1$. Take $t_{k} \in D \backslash\{0\}$ so that $t_{k} \rightarrow 0$, put $\left(x_{k}, y_{k}\right):=\left(x\left(t_{k}\right), y\left(t_{k}\right)\right)\left(\in W^{\prime}\right)$, and take $s_{k} \in \mathbb{C}^{\times}$so that $s_{k}(\operatorname{grad} \log f)\left(x_{k}\right)=y_{k}$. Then

$$
\left|s_{k}\right| \cdot\left|\operatorname{grad} f\left(x_{k}\right)\right|=\left|y_{k} f\left(x_{k}\right)\right| \leq 2\left|y_{k}\right| \cdot\left|\bar{x}\left(t_{k}\right)\right| \cdot\left|\operatorname{grad} f\left(x_{k}\right)\right| .
$$

Since $f$ is locally constant on grad $f=0\}$, and since $f\left(x_{k}\right) \neq 0$ but $f\left(x_{0}\right)=0$, $\operatorname{grad} f\left(x_{k}\right) \neq 0$ for $k \gg 0$. Hence $\left|s_{k}\right| \leq 2\left|y_{k}\right| \cdot\left|\bar{x}\left(t_{k}\right)\right|$ for $k \gg 0$ and we get the assertion. 
Proof of sublemma. We may assume that $W$ is an irreducible affine variety. By the Noether's normalization theorem, there is a finite surjective morphism $\pi: W \rightarrow k^{m}$, where $m=\operatorname{dim} W$. Put $Z=\pi\left(W \backslash W^{\prime}\right)$. Then $Z$ is a closed subset of $k^{m}$ and $\operatorname{dim} Z<m$. Let $A=k\left[T_{1}, \cdots, T_{m}\right.$ ] (the polynomial $\operatorname{ring}$ ), $B=k[W]$ (the ring of regular functions on $W$ ), and $A_{0}$ (resp. $B_{0}$ ) be the localization of $A$ (resp. $B)$ at $\pi(p)$ (resp. $p$ ). Consider $A$ (resp. $A_{0}$ ) as a subring of $B$ (resp. $B_{0}$ ) by $\pi^{*}$. Take an affine line $L$ such that $\pi(p) \in L \nsubseteq Z$. Let $P$ be the prime ideal of $A$ defining $L, P_{0}$ the prime ideal of $A_{0}$ generated by $P, Q_{0}$ a prime ideal of $B_{0}$ such that $Q_{0} \cap A_{0}=P_{0}$ (going-up theorem of Cohen-Seidenberg), $Q=Q_{0} \cap B$, and $C$ the irreducible subvariety of $W$ defined by $Q$. Then $p \in C$ and $\pi$ induces a finite surjective morphism $C \rightarrow L$. Hence $C$ is an irreducible curve with the desired property.

Lemma 2.4.4. $p(\tilde{W})=W$.

Proof. First, assume that $f$ is quasi-homogeneous, i.e., there exist $a_{i} \in$ $A(V)$ such that $\sum_{i=1}^{n} a_{i} \frac{\partial f}{\partial x_{i}}=f$. Since

$$
s \frac{\partial f}{\partial x_{j}}=y_{j} f(x)=\sum_{i=1}^{n} y_{j} a_{i} \frac{\partial f}{\partial x_{i}}
$$

holds on $\tilde{W}$, the finite $\boldsymbol{C}\left[V \times V^{\vee}\right]$-module $\sum_{i=1}^{n} \boldsymbol{C}\left[V \times V^{\vee}\right] \frac{\partial f}{\partial x_{i}}$ is stable under the multiplication by $s$. Hence $\left.s\right|_{\tilde{w}}$ is finite over $\mathbb{C}\left[V \times V^{\vee}\right]$. Hence $\left.p\right|_{\tilde{w}}$ is a finite morphism, $p(\widetilde{W})$ is Zariski closed in $V \times V^{\vee}$, and $p(\tilde{W})=W$.

In the general case, $\operatorname{let}\left(x_{0}, x\right) \in C \times V, f_{1}\left(x_{0}, x\right)=x_{0} f(x)$, and define $\tilde{W}_{1}^{\prime}, \tilde{W}_{1}$, $W_{1}^{\prime}, W_{1}$ in the same way as $\widetilde{W}^{\prime}, \widetilde{W}, W^{\prime}, W$ using $f_{1}$ instead of $f$. Then we have the commutative diagram

$$
\begin{array}{ccc}
\tilde{W}_{1} & \rightarrow & \tilde{W} \\
\downarrow & & \downarrow \\
W_{1} & \rightarrow & W
\end{array}
$$

where the morphisms are natural projections. Since $f_{1}$ is quasi-homogeneous, $\widetilde{W}_{1} \rightarrow W_{1}$ is surjective. Hence it is enough to prove that $W_{1} \rightarrow W$ is surjective. Let $(x, y)$ be an element of $W$ and let us find an element of $W_{1}$ lying above it. If $f(x) \neq 0,(x, y)=(x, s$ grad $\log f(x))$ with some $s \in \mathbb{C}$. Then $(1, x, s, s$ grad $\log f(x))$ is an element of $W_{1}$, lying above $(x, y)$. Let us assume that $f(x)=0$. Let $\left(x_{k}, y_{k}\right)=\left(x_{k}, s_{k} \operatorname{grad} \log f\left(x_{k}\right)\right) \in W^{\prime}$ and $\left(x_{k}, y_{k}\right) \rightarrow(x, y)$. By (2.4.3), we may assume that $s_{k} \rightarrow 0$. Hence 


$$
W_{1}^{\prime} \ni\left(s_{k}, x_{k}, 1, s_{k} \operatorname{grad} \log f\left(x_{k}\right)\right) \rightarrow(0, x, 1, y),
$$

and $(0, x, 1, y) \in W_{1}$, whose projection to $W$ is $(x, y)$.

Lemma 2.4.5。 $p\left(\tilde{W}_{0}\right)=W_{0}$.

Proof. For a given $(x, y) \in W_{0}$, we can find $s$ such that $(s, x, y) \in \widetilde{W}$ by (2.4.4). If $f(x)=0$, then we may assume $s=0$ by (2.4.3). If $f(x) \neq 0$, then $y=0$, and $(x, 0)=p(0, x, 0) \in p\left(\tilde{W}_{0}\right)$. Hence $W_{0} \subset p\left(\tilde{W}_{0}\right)$. Since $s \operatorname{grad} f(x)=f(x) y$ holds on $\tilde{W}, p\left(\tilde{W}_{0}\right) \subset W_{0}$.

Lemma 2.4.6. (1) The characterisic variety of the $D$-module $D[s] f^{s}$ is $W$ and $m\left(W, D[s] f^{s}\right)=1$.

(2) $\operatorname{ch}\left(D f^{\alpha}\right)=W_{0}$.

Proof. By (2.2.5), (1) follows from [13] and (2) follows from [25; appendix]. (If $f$ is quasi-homogeneous, $D[s] f^{s}=D f^{s}$ is a finitely generated $D$-module. For a general $f, D[s] f^{s}$ is also finitely generated as a $D$-module. See pages 49 and 50 of [13].)

\section{5. b-Functions}

2.5.0. There are several kinds of 'b-functions'. Let us consider relations among them. Here, in (2.5), we use the notation given in (2.1.3).

2.5.1. Let $V=\mathbb{C}^{n}, v \in V$ and $R$ be one of the rings $A, A_{v}, \tilde{A_{v}}$ or $\hat{A}_{v}$. For a given $f \in R$, we can find $P(s) \in R[s] \otimes_{A} D$ such that

$$
P(s) f^{s+1}=c(s) f^{s}
$$

with some $c(s) \in \mathbb{C}[s]-\{0\}$. (See [1] for the proof.) The totality of such polynomials $c(s)$ is an ideal of $\mathbb{C}[s]$, whose monic generator we shall denote by $B(s), B_{v}(s), \tilde{B}_{v}(s)$ or $\hat{B}_{v}(s)$ if $R=A, A_{v}, \tilde{A}_{v}$ or $\hat{A}_{v}$, respectively.

Lemma 2.5.2. If $f \in A, B(s)$ is the least common multiple of $\left\{B_{v}(s) \mid v \in V\right\}$.

Proof. Let $c(s)$ be the least common multiple of $\left\{B_{v}(s)\right\}$. Then $c(s)$ divides $B(s)$. Let us prove that $B(s)$ divides $c(s)$. Let $P_{v} f^{s+1}=c(s) f^{s}$ with $P_{v} \in A_{v}[s] \otimes_{A} D$. Multiplying the denominator of $P_{v}$, we get relations of the form $Q_{i} f^{s+1}=g_{i} c(s) f^{s}\left(Q_{i} \in D[s], g_{i} \in A, i=1,2, \cdots, N\right)$ with $\left(g_{1}, \cdots, g_{N}\right)=1$. If $\sum a_{i} g_{i}=1$ with $a_{i} \in A$, then $\sum a_{i} Q_{i} f^{s+1}=c(s) f^{s}$. Hence $B(s)$ divides $c(s)$.

Lemma 2.5.3. If $f$ is a homogeneous polynomial, $B(s)=B_{0}(s)$.

Proof. Let $P_{0} f^{s+1}=B_{0}(s) f^{s}$ with $P_{0} \in A_{0}[s] \otimes_{A} D$. The coefficients of $P_{0}$ 
are regular in some neighbourhood $U$ of 0 . Hence $B_{0}(s)$ is a multiple of $B_{v}(s)$ for any $v \in U$. Since $f$ is homogeneous, $B_{v}(s)=B_{t v}(s)$ for $t \in C^{\times}$. By these facts and by (2.5.2), $B_{0}(s)$ is a multiple of $B(s)$. Since $B_{0}(s)$ divides $B(s), B_{0}(s)=$ $B(s)$.

Lemma 2.5.4. If $f \in A_{v}, B_{v}=\tilde{B}_{v}=\hat{B}_{v}$.

Proof. Obviously, $\hat{B}_{v}$ divides $\tilde{B}_{v}$, and $\tilde{B}_{v}$ divides $B_{v}$. Let

$$
P f^{s+1}=\hat{B}_{v} f^{s}
$$

with $P \in \hat{A}_{v}[s] \otimes D$. Let $P=\sum_{|\alpha| \leq k} a_{\alpha} \partial^{\alpha}$ and $\partial^{\alpha} f^{s+1}=c_{\alpha} f^{s-l}$ with $k, l \in \mathbb{Z}, a_{\alpha} \in$ $\hat{A}_{v}[s]$ and $c_{\alpha} \in A_{v}[s]$. Here $\alpha=\left(\alpha_{1}, \cdots, \alpha_{n}\right),|\alpha|=\sum \alpha_{i}$ and $\partial^{\alpha}=\partial_{1}^{\alpha} \cdots \partial_{n}^{\alpha_{n}}$. Then, the linear equation

$$
\sum_{|\alpha| \leq k} x_{\infty} c_{\alpha}=\hat{B}_{v} f^{l}
$$

is satisfied by $\left(x_{\alpha}\right)=\left(a_{\alpha}\right)$. Since $\hat{A}_{v}[s]$ is faithfully flat over $A_{v}[s]$ (cf. (2.1.3)), there is a solution of (2.5.6) in $A_{v}[s]$. (Cf. [3; Chap.1, §3, Prop.13].) Hence we can find $P \in A_{v}[s] \otimes D$ which satisfies (2.5.5). Hence $B_{v}$ divides $\hat{B}_{v}$.

Lemma 2.5.7. Let $V^{v}$ be the dual space of $V$, and $p=\left(v, v^{v}\right) \in W_{0}$. (See (2.4.1) for the definition of $W_{0}$.) If $Q \in \tilde{A_{v}} \otimes D$ is invertible in $E_{p}$ and satisfies $Q f^{s+1}=b_{p}(s) f^{s}$ with $b_{p}(s) \in \mathbb{C}[s]$, then $b_{p}(s)$ is a non-zero consiant multiple of $\tilde{B}_{v}(s)$.

Proof. Let $E_{p}[s] f^{s}=E_{p} \otimes_{D} D[s] f^{s} . \quad$ By $(2.2 .5)$ and $(2.4 .6,(2))$,

$$
0 \neq E_{p} \otimes_{D}\left(D f^{\alpha}\right)=\frac{E_{p} \otimes_{D} D[s] f^{s}}{E_{p} \otimes_{D}(s-\alpha) D[s] f^{s}}=\frac{E_{p}[s] f^{s}}{(s-\alpha) E_{p}[s] f^{s}}
$$

for any $\alpha \in C$. (Recall that $E_{p}$ is flat over D.) Hence

$$
(s-\alpha) E_{p}[s] f^{s} \subsetneq E_{p}[s] f^{s} \text { for any } \alpha \in C .
$$

For any $\alpha(s) \in \mathbb{C}[s]-\{0\}, a(s) D[s] f^{s} \simeq D[s] f^{s}$ as $D$-modules. Hence

$$
a(s) E_{p}[s] f^{s} \simeq E_{p}[s] f^{s} \neq 0 .
$$

By (2.5.8) and (2.5.9), $E_{p}[s] f^{s}$ is faithfully flat over $\mathbb{C}[s]$. (See $\S 2$, Prop.3, (2) and $\S 3$, Prop.1 of $\left[3\right.$; Chap.1].) Let $b_{p}(s)=c(s) \tilde{B}_{v}(s)$. If $c(s) \notin C^{\times}$, then $b_{p}(s)$ $C[s] \subsetneq \tilde{B}_{v}(s) C[s]$ and

$$
b_{p}(s) E_{p}[s] f^{s} \subsetneq \tilde{B}_{v}(s) E_{p}[s] f^{s} \subset E_{p}[s] f^{s+1} .
$$

On the other hand, since $Q$ is invertible in $E_{p}$, 


$$
b_{p}(s) E_{p}[s] f^{s}=E_{p}[s] Q f^{s+1}=E_{p}[s] f^{s+1}
$$

Hence $c(s) \in \mathbb{C}^{\times}$.

Corollary 2.5.10. If $f$ is a relatively invariant polynomial of a prehomogeneous vector space, then $b_{0}^{-1} b(s)=B(s)=B_{0}(s)=\tilde{B}_{0}(s)=\hat{B}_{0}(s)$. (See (1.6) for $b_{0}$ and $b(s)$.)

Proof. Since $f$ is a homogeneous polynomial by $(1.3,(2))$, we have $B(s)=$ $B_{0}(s)$ by (2.5.3). By (2.5.7), it is enough to prove that there exists a point $v^{\vee} \in$ $V^{\vee}$ such that $p=\left(0, v^{\vee}\right) \in W_{0}$ and $f^{\vee}$ (grad) is invertible in $E_{p}$. (Cf. (1.6). Here we use the notation in the first section.) Hence it is enough to prove that

$$
\{0\} \times O_{1}^{\vee} \subset W_{0} \text {. }
$$

For $v \in \Omega,(\varepsilon v, \varepsilon F(\varepsilon v))=(\varepsilon v, F(v)) \rightarrow(0, F(v)),(\varepsilon \rightarrow 0)$. Hence we get (2.5.11). (Cf. $(1.18,(2))$.)

Corollary 2.5.12. The b-function is of the form

$$
b(s)=b_{0} \mathbb{M}_{j=1}^{d}\left(s+\alpha_{j}\right), \quad d=\operatorname{deg} f,
$$

where $\alpha_{j}$ 's are positive rational numbers.

Proof. The assertion follows from [13] and (2.5.10).

\subsection{Dualiety}

Lemma 2.6.1. Let $(R, m)$ be a regular local ring of dimension $r$, and $M$ an $R$-module of finite length. (See [29] for regular local rings.) Then

$$
\operatorname{Ext}_{R}^{j}(M, R)=0 \text { for } j \neq r
$$

and

$$
\text { length }_{R}\left(\operatorname{Ext}_{R}^{r}(M, R)\right)=\text { length }_{R}(M) .
$$

Proof. If length $(M)=1$, i.e., $M=R / m$, a free resolution of $M$ can be constructed explicitly and $\operatorname{Ext}_{R}^{j}(M, R)$ can be calculated. (Consider the complex $K\left(x_{1}, \cdots, x_{r} ; M\right)$ given in $[29 ; \mathbb{I V}-4]$, where $\left\{x_{1}, \cdots, x_{r}\right\}$ is a regular system of parameters of $R[29 ; \mathbb{I V}-40]$.) Our assertion follows from this special case by an induction on the length of $M$.

Lemma 2.6.2. Let $M$ be a finitely generated (left) $D(U)$-module, $C$ an irreducible component of $\operatorname{ch}(M)$, and $s=\operatorname{codim}_{U \times V^{v}}(C)$. Then 


$$
m\left(C, \operatorname{Ext}_{D}^{s}(M, D) \otimes_{A} \Omega^{-1}\right)=m(C, M) .
$$

(See (2.1.2) for $\otimes \Omega^{-1}$, and (2.2.4) for $m(C,-)$.)

Proof. Let $(M, F)$ be a good filtration of $M$ (cf. (2.2.3)), and

$$
\stackrel{d}{\rightarrow}\left(M_{1}, F\right) \stackrel{d}{\rightarrow}\left(M_{0}, F\right) \stackrel{\varepsilon}{\rightarrow}(M, F) \rightarrow 0
$$

a filtered complex such that

(1) $M_{\mathrm{o}}:=\left(\cdots \stackrel{d}{\rightarrow} M_{1} \stackrel{d}{\rightarrow} M_{0} \stackrel{\varepsilon}{\rightarrow} M \rightarrow 0\right)$ is exact,

(2) $\ldots \stackrel{\operatorname{gr}(d)}{\longrightarrow} \operatorname{gr}\left(M_{1}\right) \stackrel{\operatorname{gr}(d)}{\longrightarrow} \operatorname{gr}\left(M_{0}\right) \stackrel{\operatorname{gr}(\varepsilon)}{\longrightarrow} \operatorname{gr}(M) \rightarrow 0$ is exact,

(3) each $M_{j}$ is finitely generated free $D$-module, say, $M_{j}=\bigoplus_{1 \leq k \leq r(j)} D u_{j, k}$, and

(4) $\quad F_{i} M_{j}=\bigoplus_{1 \leq k \leq r(j)} D_{i-s(j, k)} u_{j, k}$ with some $s(j, k)$.

Such a filtered complex exists for any $(M, F)[1$; Chap. 2, 3.11]. Let

$$
F_{i} \operatorname{Hom}_{D}\left(M_{\circ}, D\right)=\left\{h \in \operatorname{Hom}_{D}\left(M_{\circ}, D\right) \mid h\left(F_{k} M_{\circ}\right) \subset D_{i+k} \text { for any } k\right\} .
$$

For each $j,\left(\operatorname{Hom}_{D}\left(M_{j}, D\right), F\right)$ is a good filtration and

$$
\operatorname{gr} \operatorname{Hom}_{D}\left(M_{j}, D\right)=\operatorname{Hom}_{\mathrm{gr}(D)}\left(\operatorname{gr}\left(M_{j}\right), \operatorname{gr}(D)\right) \text {. }
$$

Let $\left(\operatorname{Ext}_{D}^{j}(M, D), F\right)$ be the filtration induced from $\left(\operatorname{Hom}\left(M_{\bullet}, D\right), F\right)$. Consider the spectral sequence $\left(E_{r}^{p q}\right)$ associated to the filtered complex $\left(\operatorname{Hom}\left(M_{\bullet}, D\right), F\right)$, and let $E_{r}^{t}=\bigoplus_{p+q=t} E_{r}^{p q}$. Then

$$
\begin{aligned}
& E_{0}^{t}=\operatorname{Hom}_{\operatorname{gr}(D)}\left(\operatorname{gr}\left(M_{t}\right), \operatorname{gr}(D)\right), \\
& E_{1}^{t}=\operatorname{Ext}_{\mathrm{gr}^{t}(D)}(\operatorname{gr}(M), \operatorname{gr}(D)), \text { and } \\
& E_{\infty}^{t}=\operatorname{gr}_{\operatorname{Ext}_{D}^{t}(M, D) .}
\end{aligned}
$$

Let $S=\left\{a \in \mathbb{C}\left[U \times V^{\vee}\right] \mid a \neq 0\right.$ on $\left.C\right\}$. Then by (2.6.1),

$$
S^{-1} E_{1}^{t}=\operatorname{Ext}_{S^{-1} \operatorname{gr}(D)}\left(S^{-1} \operatorname{gr}(M), S^{-1} \operatorname{gr}(D)\right)=0
$$

for $t \neq s$. (Note that $S^{-1} \operatorname{gr}(D)$ is a regular local ring of dimension $s$.) Hence the spectral sequence $\left(S^{-1} E_{r}^{p q}\right)$ degenerates at $E_{1}$-terms, and

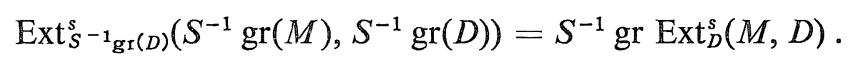

By definition, $m\left(C, \operatorname{Ext}_{D}^{s}(M, D) \otimes \Omega^{-1}\right)$ is the length of the right hand side of this equality. On the other hand, by (2.6.1), the length of the left hand side is equal to that of $S^{-1} \operatorname{gr}(M)$, which is $m(C, M)$. 
2.6.3. If $M$ is a left $D[s, t]$-module (cf. (2.3.5)), then we can define a left $D[s, t]$-module structure of $\operatorname{Hom}_{D}(M, D)$ by

$$
(P \varphi)(u)=\varphi(u) P^{*}, \quad(s \varphi)(u)=-\varphi(s u) \quad \text { and } \quad(t \varphi)(u)=\varphi(t u),
$$

for $\varphi \in \operatorname{Hom}_{D}(M, D), u \in M$ and $P \in D$. (See (2.1.2) for $P^{*}$. Note that our definition of the $D[s, t]$-module structure here depends on the choice of the coordinate system. In order to make the description free from the coordinate system, it suffices to consider $\operatorname{Hom}_{D}(M, D) \otimes_{A} \Omega^{-1}$ etc.) Hence $\operatorname{Ext}_{D}^{j}(M, D)$ has a left $D[s, t]$-module structure. Let $N=D[s] f^{s}, N(\alpha)=D f^{\alpha}$ (cf. (2.3.1)), $N^{*}=$ $\operatorname{Ext}_{D}^{n-1}(N, D)$ and $N(\alpha)^{*}=\operatorname{Ext}_{D}^{n}(N(\alpha), D)$. In general, set $M^{*}=\operatorname{Ext}_{D}^{n}(M, D)$ for a holonomic $D$-module $M$. (See (2.2.3) for the definition of a holonomic $D$ module.) Since $N$ is a left $D[s, t]$-module by $(2.3 .5), N^{*}$ is also a left $D[s, t]$ module.

Lemma 2.6.4. (1) $\operatorname{Ext}_{D}^{j}(N, D)=0$ for $j \neq n-1$.

(2) If a $D$-module $M$ is holonomic, then $\operatorname{Ext}_{D}^{j}(M, D)=0$ for $j \neq n$.

(3) If $\alpha_{+} \in A_{+}(B), \alpha_{-} \in A_{-}(B)$ and $\alpha_{+}+\alpha_{-} \equiv 0 \bmod \mathbb{Z}_{\text {, then }} N\left(\alpha_{+}\right)^{*} \simeq N\left(\alpha_{-}\right)$ and $N\left(\alpha_{+}\right) \simeq N\left(\alpha_{-}\right)^{*}$.

(See (2.3.6) for $A_{+}$and $A_{-}$, and (2.5.1) for $B$. )

Proof. (1) and (2) are essentially proved in [13]. In order to prove (3), it is enough to prove that

$$
N\left(\alpha_{+}+k\right) \simeq N\left(\alpha_{-}-l\right)^{*}
$$

for some non-negative integers $k$ and $l$. (Cf. (2.3.9).) We prove this in several steps.

2.6.5. First step. First, let us define a $D[s, t]$-module homomorphism $r: N^{*} \rightarrow N$ on the open set $U=V-f^{-1}(0)$. Here, in (2.6.5), $A$ and $D$ always stand for $A(U)$ and $D(U)$.

Let $\theta=\bigoplus_{1 \leq i \leq n} A[s] v_{i}$ and define a free resolution of the $D[s]$-module $A[s]$

$$
0 \rightarrow D[s] \otimes_{A[s]} \bigwedge^{n} \theta \stackrel{\partial}{\rightarrow} \ldots \stackrel{\partial}{\rightarrow} D[s] \otimes_{A[s]} \stackrel{0}{\wedge} \stackrel{\varepsilon}{\rightarrow} A[s] \rightarrow 0
$$

by

$$
\partial\left(P \otimes v_{1} \wedge \cdots \wedge v_{k}\right)=\sum_{i=1}^{k}(-1)^{i-1} P \partial_{i} \otimes\left(v_{1} \wedge \cdots \wedge v_{i-1} \wedge v_{i+1} \wedge \cdots \wedge v_{k}\right)
$$

and

$$
\varepsilon(P)=P \cdot 1
$$


Since $N=D[s] f^{s}$ is a free $A[s]$-module generated by $f^{s},\left(D[s] \otimes \bigwedge^{k} \theta \otimes N, \partial \otimes N\right)$ gives a free resolution of the $D[s]$-module $N$ in the following manner. The $D[s]$-module structure of $D[s] \otimes \bigwedge^{k} \theta \otimes N$ is given by

$$
\partial_{i}\left(P \otimes w \otimes f^{s}\right)=\partial_{i} P \otimes w \otimes f^{s}+P \otimes w \otimes \partial_{i}\left(f^{s}\right) .
$$

The set $\left\{1 \otimes\left(v_{i_{1}} \wedge \cdots \wedge v_{i_{k}}\right) \otimes f^{s} \mid 1 \leq i_{1}<\cdots<i_{k} \leq n\right\}$ gives a free basis of the $D[s]$-module $D[s] \otimes \wedge^{k} \theta \otimes N$. Consider the complex

$$
\left(\operatorname{Hom}_{D}\left(D[s] \otimes \bigwedge^{k} \theta \otimes N, D\right), \delta\right),
$$

where $\delta$ is the morphism induced by $\partial \otimes N$. Let

$$
\begin{aligned}
& w=v_{1} \wedge \cdots \wedge v_{n}, \\
& w_{i}=v_{1} \wedge \cdots \wedge v_{i-1} \wedge v_{i+1} \wedge \cdots \wedge v_{n}, \\
& w_{i j}=v_{1} \wedge \cdots \wedge v_{i-1} \wedge v_{i+1} \wedge \cdots \wedge v_{j-1} \wedge v_{j+1} \wedge \cdots \wedge v_{n}, \\
& w_{j i}=-w_{i j}
\end{aligned}
$$

for $i<j$, and $w_{i i}=0$. For a given $\varphi \in \operatorname{Hom}_{D}\left(D[s] \otimes \bigwedge^{k} \theta \otimes N, D\right)$, let

$$
\begin{aligned}
P_{l} & =\varphi\left(1 \otimes w \otimes s^{l} f^{s}\right), \\
Q_{i, l} & =\varphi\left(1 \otimes w_{i} \otimes s^{l} f^{s}\right), \\
R_{i, j, l} & =\varphi\left(1 \otimes w_{i, j} \otimes s^{l} f^{s}\right),
\end{aligned}
$$

and identify $\varphi$ with $P=\left(P_{l}\right), Q=\left(Q_{i l}\right), R=\left(R_{i j l}\right)$ if $k=n, n-1, n-2$, respectively. If we set $f_{i}=\frac{\partial f}{\partial x_{i}}$, then by the identities

$$
\partial_{i} \otimes w_{i} \otimes s^{l} f^{s}=\partial_{i}\left(1 \otimes w_{i} \otimes s^{l} f^{s}\right)-1 \otimes w_{i} \otimes \partial_{i}\left(s^{l} f^{s}\right) \text { etc. }
$$

we get

$$
\begin{aligned}
& (\delta Q)_{l}=\sum_{i=1}^{n}(-1)^{i-1}\left(\partial_{i} Q_{i, l}-\left(f_{i} / f\right) Q_{i, l+1}\right), \\
& (\delta R)_{j, l}=\sum_{i=1}^{n}(-1)^{i-1}\left(\partial_{i} R_{i, j, l}-\left(f_{i} / f\right) R_{i, j, l+1}\right), \\
& R_{i, j, l}+R_{j, i, l}=0 .
\end{aligned}
$$

The $D[s, t]$-module structure of $N$ can be lifted to $D[s] \otimes \bigwedge^{k} \theta \otimes N$;

$$
t\left(P(s) \otimes\left(v_{i_{1}} \wedge \cdots \wedge v_{i_{k}}\right) \otimes Q(s) f^{s}\right)=P(s+1) \otimes\left(v_{i_{1}} \wedge \cdots \wedge v_{i_{k}}\right) \otimes Q(s+1) f \cdot f^{s} .
$$

We give a left $D[s, t]$-module structure of $\operatorname{Hom}_{D}\left(D[s] \otimes \bigwedge^{k} \theta \otimes N, D\right)$ by $(s \varphi)(x)$ $=-\varphi(s x),(t \varphi)(x)=\varphi(t x)$ and $(P \varphi)(x)=\varphi(x) P^{*}$ for $P \in D$. (See (2.1.2) for $P^{*}$.) Then 


$$
(s Q)_{i, l}=-Q_{i, l+1}, \quad(t Q)_{i, 0}=f Q_{i, 0},
$$

and $\delta$ 's are compatible with the left $\mathbb{C}[s, t]$-module structures. Define a $D$ module homomorphism $r:\{Q\} \rightarrow \mathbb{D}[s] f^{s}$ by

$$
r(Q)=\sum_{i=1}^{n}(-1)^{i-1} Q_{i, 0}^{*}\left(f_{i} / f\right) f^{s} .
$$

If $\delta Q=0$, we can show that $r(s Q)=s \gamma(Q), r(t Q)=\operatorname{tr}(Q)$ and $r(\delta R)=0$ by using (2.6.6) and (2.6.7). Hence we get a $D[s, t]$-module homomorphism $\gamma: \operatorname{Ext}_{D}^{n-1}(N, D) \otimes_{A} \Omega^{-1} \rightarrow N$. (Recall that $D=D\left(V-f^{-1}(0)\right)$.)

2.6.8. Second step. Next, let us show that the morphism $r: N^{*} \rightarrow N$ constructed in the first step is not identically zero if $f$ is quasi-homogeneous. We continue to write $A$ and $D$ for $A(U)$ and $D(U)$. Since $f$ is assumed to be quasihomogeneous, there exist $a_{i}^{\prime} \in A$ such that $\sum_{i=1}^{n} f_{i} a_{i}^{\prime}=f$. Let $(-1)^{i} a_{i}^{\prime} f^{-1}=a_{i}$. (Note that $f$ is invertible in $A=A\left(V-f^{-1}(0)\right)$.) Then $\sum_{i=1}^{n}(-1)^{i} f_{i} a_{i}=1$. Since $\delta Q=0$ if and only if $\sum_{i=1}^{n}(-1)^{i} \partial_{i} Q_{i, l}=\sum_{i=1}^{n}(-1)^{i} f_{i} Q_{i, l+1}$ for any $l \geq 0$ (cf. (2.6.6)), and since $\sum_{i=1}^{n}(-1)^{i} f_{i} \circ a_{i} T=T$ for any $T \in D$, the morphism ker $\delta \rightarrow \mathbb{D}^{n}$ defined by $Q \rightarrow\left(Q_{i, 0}\right)_{1 \leq i \leq n}$ is surjective. Hence $\gamma \neq 0$.

2.6.9. Thired step。 Here, in (2.6.9), $A$ and $D$ stand for $A(V)$ and $D(V)$, and we assume $f$ to be quasi-homogeneous. Since $N=D f^{s}, N^{*}$ is generated as a $D$-module by a finite generator system, say $u_{1}, \cdots, u_{p}$. (Actually, this is always the case, even without assuming $f$ to be quasi-homogeneous.) Their images by $r$ can be expressed as $r\left(u_{i}\right)=P_{i}(s) f^{-m} \circ f^{s}$ with $\mathbb{P}_{i}(s) \in D[s]$ if $m$ is a sufficiently large integer. Then we can define a $D[s, t]$-module homomorphism $\gamma_{1}: N^{*} \rightarrow N$ by $\gamma_{1}\left(u_{i}\right)=P_{i}(s+m) f^{s}$. Since $\gamma_{1} \neq 0$ by $(2.6 .8)$, since $\operatorname{cln}\left(N^{*}\right)=\operatorname{chl}(N)=W$ by (2.4.6) and (2.6.2), and since $N$ does not have a holonomic submodule other than 0 by [13; Theorem (2.12) and Corollary (5.12)], ker $\gamma_{1}$ and coker $\gamma_{1}$ are both holonomic $D[s, t]$-modules by (2.2.6). Hence by [13; Prop. 5.11], $t^{k}$ ker $\gamma_{1}=0$ and $t^{k}$ coker $\gamma_{1}=0$ for a sufficiently large integer $k$. If $t^{k} u \in \operatorname{ker} \gamma_{1} \cap$ $t^{k} N^{*}$, then $t^{k} \gamma_{1}(u)=0$. Since $t: N \rightarrow N$ is injective, $\gamma_{1}(u)=0$ and $t^{k} u \in t^{k} \operatorname{ker} \gamma_{1}$ $=0$. Hence $r_{1}: t^{k} N^{*} \rightarrow t^{k} N$ is injective. Since $t^{k} N \subset r_{1}\left(N^{*}\right)$, we get injective morphisms $t^{2 k} N \rightarrow t^{k} N^{*} \rightarrow t^{k} N$, from which we get

$$
\frac{t^{2 k} N^{*}}{(s-\alpha) t^{2 k} N^{*}} \stackrel{a}{\rightarrow} \frac{t^{2 k} N}{(s-\alpha) t^{2 k} N} \stackrel{b}{\rightarrow} \frac{t^{k} N^{*}}{(s-\alpha) t^{k} N^{*}} \stackrel{c}{\rightarrow} \frac{t^{k} N}{(s-\alpha) t^{k} N} .
$$

Since 


$$
\frac{t^{k} N}{(s-\alpha) t^{k} N}=\frac{t^{k} N}{t^{k}(s-\alpha-k) N}=N(\alpha+k)
$$

and $c b$ can be identified with the natural morphism $N(\alpha+2 k) \rightarrow N(\alpha+k), c b$ is an isomorphism if $k$ is sufficiently large (cf. (2.3.9)). Hence $b$ is injective. Since $0 \rightarrow N \stackrel{t}{\rightarrow} N \rightarrow N / t N \rightarrow 0$ is exact, and $N / t N$ is holonomic, $0 \rightarrow N^{*} \stackrel{t}{\rightarrow} N^{*} \rightarrow$ $(N / t N)^{*} \rightarrow 0$ is exact and, especially, $N^{*} \stackrel{t}{\rightarrow} N^{*}$ is injective. Cf. $(2.6 .4,(2))$. (Note that $(N / t N)^{*}=\operatorname{Ext}_{D}^{n}(N / t N, D)$.) In the same way, we get an exact sequence $0 \rightarrow N^{*} \stackrel{-s-\alpha}{\longrightarrow} N^{*} \rightarrow N(\alpha)^{*} \rightarrow 0$. Hence

$$
\frac{t^{k} N^{*}}{(s-\alpha) t^{k} N^{*}}=\frac{N^{*}}{(s-\alpha-k) N^{*}}=N(-\alpha-k)^{*},
$$

and $b a$ can be identified with the morphism $N(-\alpha-2 k)^{*} \rightarrow N(-\alpha-k)^{*}$ induced by $N(-\alpha-k) \rightarrow N(-\alpha-2 k)$. Hence $b a$ is an isomorphism if $k$ is sufficiently large (cf. (2.3.9)), and $b$ is surjective. Thus we get an isomorphism $N(\alpha+2 k)$ $\stackrel{b}{\rightarrow} N(-\alpha-k)^{*}$, assuming $k$ to be sufficiently large. Hence by (2.3.9), we get the assertion for quasi-homogeneous $f$.

2.6.10. End of the proof. Let $x_{0}$ be a new variable, $D_{0}=D(\mathbb{C})$ and $D_{1}=$ $D(C \times V)$. Since $D_{1}\left(f x_{0}\right)^{\alpha}$ is naturally isomorphic to $D f^{\alpha} \otimes_{C} D_{0} x_{0}^{\alpha}$,

$$
\left(D_{1}\left(f x_{0}\right)^{\alpha}\right)^{*}=\left(D f^{\alpha}\right)^{*} \otimes_{C}\left(D_{0} x_{0}^{\alpha}\right)^{*} .
$$

By (2.6.9) and (2.6.11), we get an isomorphism

$$
r_{1}:\left(D f^{\alpha+k}\right) \otimes_{C}\left(D_{0} x_{0}^{\alpha+k}\right) \rightarrow\left(D^{-\alpha-k}\right)^{*} \otimes_{C}\left(D_{0} x_{0}^{\alpha+k}\right),
$$

if $k$ is a sufficiently large integer. Since $\left(x_{0} \frac{\partial}{\partial x_{0}}-\alpha-k\right) r_{1}\left(u \otimes x_{0}^{\alpha+k}\right)=0$ for any $u \in D f^{\alpha+k}$, we can uniquely express $r_{1}\left(u \otimes x_{0}^{\alpha+k}\right)$ as $r(u) \otimes x_{0}^{\alpha+k}$ with $r(u) \in$ $\left(D f^{-\alpha-k}\right)^{*}$. By this $r, D f^{\alpha+k} \simeq\left(D f^{-\alpha-k}\right)^{*}$.

Remark 2.6.12. As far as $f^{-1}(0) \neq \phi, N^{*}$ is not isomorphic to $N$ as $D[s]-$ modules. In fact, if $N^{*} \simeq N$, the following two exact sequences coincide;

$$
\begin{aligned}
0 \rightarrow & N^{*} \stackrel{(s-\alpha)^{*}}{\longrightarrow} N^{*} \rightarrow N(\alpha)^{*} \rightarrow 0 \\
& \|\quad\| \quad \| \quad N \quad \stackrel{-s-\alpha}{\longrightarrow} N \rightarrow N(-\alpha) \rightarrow 0 .
\end{aligned}
$$

By (2.5.12) and (2.3.9), $N(0) \simeq N(k)$ for any positive integer $k$, and as their dual, $N(0) \simeq N(-k)$. Since $\tilde{A_{0}} \otimes_{A} D(\mathbb{C}) x^{0}$ is not isomorphic to $\tilde{A_{0}} \otimes_{A} D(\mathbb{C}) x^{-1}$, 
$N(0) \simeq N(-1)$ implies $f^{-1}(0)=\phi$

\subsection{Fourren transformation}

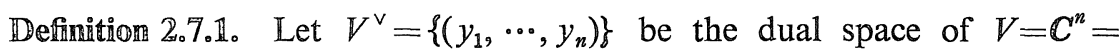
$\left\{\left(x_{1}, \cdots, x_{n}\right)\right\}$. Define an algebra isomorphism $\mathscr{F}: D(V) \rightarrow D\left(V^{\vee}\right)$ by

$$
\mathscr{F}\left(x_{i}\right)=\sqrt{-1} \frac{\partial}{\partial y_{i}} \text { and } \mathscr{F}\left(\frac{\partial}{\partial x_{i}}\right)=\sqrt{-1} y_{i} .
$$

For a $D(V)$-module $M$, define a $D\left(V^{\vee}\right)$-module $\mathscr{F}(M)$ as follows. As an additive group $\mathscr{F}(M)=M$. Denote an element $u$ of $M$ by $\mathscr{F}(u)$ if $u$ is considered as an element of $\mathscr{H}(M)$. The $D\left(V^{\vee}\right)$-module structure of $\mathscr{F}(M)$ is defined by $\mathscr{F}(P) \mathscr{F}(u)=\mathscr{F}(P u)$ for $P \in D(V)$ and $u \in M$. The $D\left(V^{\vee}\right)$-module $\mathscr{F}(M)$ is called the Fourier transform of $M$. If $M$ has a $D(V)[s]$-module structure or a $D(V)[s, t]$-module structure, we define the actions of $s$ and $t$ on $\mathscr{F}(M)$ by $s \mathscr{F}(u)=\mathscr{F}(s u), t \mathscr{F}(u)=\mathscr{F}(t u) . \quad($ See $(2.3 .5)$ for $s$ and $t$.)

Lemma 2.7.2。([12])。 Let $X=\sum_{i=1}^{n} x_{i} \partial_{i} \in D(V), M$ be a $D$-module and $u$ an element of $M$ such that $X u=c u$ with some $c \in \mathbb{C}$. Then ch $\mathscr{F}(D u)=-\sqrt{-1}$ $\operatorname{ch}(D u)\left(\subset V \times V^{\vee}\right)$.

\subsection{Regular holomomic $\mathbb{D}$-module}

2.8.1. For a smooth quasi-projective (algebraic) variety $X$ of dimension $r$ over $\mathbb{C}$, let $\mathcal{O}=\mathcal{O}_{X}$ be the sheaf of regular functions, $\Omega=\Omega_{X}$ the sheaf of regular $r$-forms, $\mathscr{D}=\mathscr{D}_{X}$ the sheaf of algebraic differential operators, $\mathcal{O}^{a n}=\mathcal{O}_{X}^{a n}$ the sheaf of holomorphic functions, and for any $\mathcal{O}$-module $\mathscr{M}, \mathscr{M}^{a_{n}}=\mathcal{O}^{a_{n}} \otimes_{\mathcal{O}} \mathscr{M}$. If $X$ is a Zariski open subset of $\mathbb{C}^{n}$ and $M$ is an $A(X)$-module, we denote the $\mathcal{O}_{X-}^{a n}$ module $\mathcal{O}_{X}^{a n} \otimes_{A(X)} M$ by $M^{a n}$. All the definitions and results concerning $D$ modules given in (2.1)-(2.6) can be generalized to $\mathscr{D}$-modules.

2.8.2. As for the definition of the regularity of a holonomic $\mathscr{D}$-module, we adopt the one given in [11; chap. 4]. (Because of the length, we do not repeat it here.) Note that a $\mathscr{D}$-module $\mathscr{M}$ on an affine line $\mathbb{C}$ defined by a single equation

$$
\sum P_{j}(x)\left(\frac{d}{d x}\right)^{j} u=0
$$

is regular in our sense if and only if $(\mathrm{F})$ does not have an irregular singularity at any point of $\mathbb{C} \cup\{\infty\}$, i.e., $(\mathbb{F})$ is of Fuchsian type. In the case where $X$ is an affine open subset of $\mathbb{C}^{n}$, we call a (left) $D(X)$-module $M$ regular, if $\mathcal{O}_{X} \otimes_{A(X)} M$ 
is regular.

2.8.3. For a left $\mathscr{D}^{a n}$-module $\mathscr{M}$, let

$$
\begin{aligned}
& \operatorname{Sol}(\mathscr{M})=R \operatorname{Hom} \mathscr{D}^{a n}\left(\mathscr{M}, \mathcal{O}^{a n}\right), \text { and } \\
& \operatorname{DR}(\mathscr{M})=\Omega^{a n} \otimes_{\mathscr{D}^{a n}}^{L} \mathscr{M}[-n]=R \operatorname{Hom} \mathscr{D}^{a n}\left(\mathcal{O}^{a n}, \mathscr{M}\right) .
\end{aligned}
$$

Here Hom denotes the sheaf of local homomorphisms, and, $R$ and $L$ means the right and left derived functors. See [33] for the generalities concerning the derived categories. For a left $\mathscr{D}$-module $\mathscr{M}$, we write $\operatorname{Sol}(\mathscr{M})($ resp. $\operatorname{DR}(\mathscr{M}))$ for $\operatorname{Sol}\left(\mathscr{M}^{a n}\right)$ (resp. DR( $\left(\mathscr{M}^{a n}\right)$ ). If $X$ is a Zariski open subset of $\mathbb{C}^{n}$, and $M$ is a left $D(X)$-module, then we write $\operatorname{Sol}(M)$ (resp. $\operatorname{DR}(M)$ ) for $\operatorname{Sol}\left(M^{a n}\right)$ (resp. $\left.\operatorname{DR}\left(M^{a n}\right)\right)$.

Lemma 2.8.4. (1) Let

$$
0 \rightarrow \mathscr{M}_{1} \rightarrow \mathscr{M}_{2} \rightarrow \mathscr{M}_{3} \rightarrow 0
$$

be an exact sequence of holonomic $\mathscr{D}$-modules. Then $\mathscr{M}_{1}$ and $\mathscr{M}_{3}$ are regular if and only if $\mathscr{M}_{2}$ is regular.

(2) A holonomic $\mathscr{D}$-module is regular if and only if its composition factors are regular. (As is shown by (2.2.6), a holonomic D-module is of finite length.)

Lemma 2.8.5. Let $\Re$ be a $\mathscr{D}[s, t]$-module (cf. (2.3.5)) such that

(1) for any $\alpha \in \mathbb{C}, \mathcal{Z}(\alpha)=\Re /(s-\alpha) \Re$ is holonomic,

(2) $t: \Re \rightarrow \Re$ is injective, and

(3) $\Re / t \mathscr{T}$ is holonomic.

Then the composition factors (up to permutation) of $\mathscr{R}(\alpha)$ depends only on $\alpha$ $\bmod \mathbb{Z}$.

Proof. With some homomorphism $\varphi$, the following diagram becomes commutative;

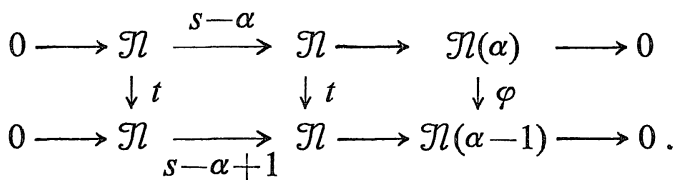

By the snake lemma [3; Chap. 1, $\$ 1$, Prop. 2],

$$
0 \rightarrow \operatorname{ker} \varphi \rightarrow \Re / t \Re \rightarrow \Re / t \Re \rightarrow \operatorname{coker} \varphi \rightarrow 0
$$

is exact. By (3), the composition factors of $\operatorname{ker} \varphi$ and coker $\varphi$ coincide (up 
to permutation) with each other. Since

$$
0 \rightarrow \operatorname{ker} \varphi \rightarrow \Re(\alpha) \rightarrow \Re(\alpha-1) \rightarrow \operatorname{coker} \varphi \rightarrow 0
$$

is also exact, the composition factors of $\mathscr{R}(\alpha)$ and $\mathscr{R}(\alpha-1)$ coincide with each other.

Lemma 2.8.6. Let $\mathscr{M}$ be a regular holonomic $\mathscr{D}$-module on a variety $X$ and $u \in \mathscr{M}$. Then $\mathscr{D}\left(f^{\alpha} u\right)$ is regular holonomic. Especially, $\mathscr{D} f^{\alpha}$ is regular holonomic. (See (2.3.1) for $\mathscr{D} f^{a}$ and (2.3.2) for $\mathscr{D}\left(f^{a} u\right)$.)

Proof. By [14; Theorem 2.5], $\mathscr{D}\left(f^{\alpha} u\right)$ is holonomic. By [14; Theorem 2.7], (2.3.7) holds with some $P(s)$ and $c(s)$. Consider $A_{ \pm}$of this polynomial $c(s)$. (See (2.3.6) for $A_{ \pm}$). Take an integer $k$ so that $\alpha+k \in A_{-} . \quad B y(2.8 .4)$ and (2.8.5), we may replace $\alpha$ with $\alpha+k$. Thus we may assume $\alpha \in A_{-}$from the beginning. By (2.3.8), $\mathscr{D}\left(f^{\alpha} u\right)=\mathscr{D}\left(f^{\alpha} u\right)\left[f^{-1}\right]$. We have $\mathscr{D}$-isomorphism $\mathscr{D}\left(f^{\alpha} u\right)=\mathscr{D}\left(f^{\alpha} u\right)\left[f^{-1}\right] \rightarrow\left(\mathscr{D} f^{\alpha} \otimes_{\mathcal{O}} \mathscr{D} u\right)\left[f^{-1}\right]$ defined by $P f^{\alpha} u \rightarrow P\left(f^{\alpha} \otimes u\right)$. Since $\mathscr{D} f^{\alpha} \otimes_{\mathcal{O}} \mathscr{D} u=\Delta^{*}\left(\mathscr{D} f^{\alpha} \bigotimes \mathscr{D} u\right)$, where $\Delta: X \rightarrow X \times X$ is the diagonal embedding, it is enough to prove the regularity of $\mathscr{D} f^{\infty}$. (Here we used 111 ; Chap. 4, 2.2.2] which asserts that the regularity is preserved by several functors.) Let $\bar{X}$ be a smooth completion of $X$. It suffices to show that the $\mathscr{D}_{X}^{a n}$-module $\mathscr{D}_{X}^{a n} f^{\infty}$ can be extended to a regular holonomic $\mathscr{D}_{\bar{X}}^{a n}$-module. (By [11; Chap. 4, 1.1.3

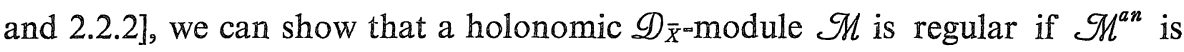
regular.) By a proper modification of $\bar{X}$, we may assume that the rational morphism $f: \bar{X} \rightarrow \mathbb{P}^{1}$ is regular on the whole $\bar{X}[10]$. Then $\mathscr{D}_{\bar{X}}^{a n} f^{\alpha}$ is regular in a neighbourhood of $f^{-1}(0)$ by [16; Theorem 2.2]. It is also regular in a neighbourhood of $f^{-1}(\infty)$, since $\mathscr{D}_{\bar{X}}^{a n} f^{\alpha}=\mathscr{D}_{\bar{X}}^{a n}\left(f^{-1}\right)^{-\alpha}$ and $f^{-1}$ is a regular function there.

\section{§3。 DD-modunes amall Prehomogemeous Vector Spaces}

3.0. In this section, first we give a description of the Fourier transform of the system of differential equations $D f^{a}$ satisfied by the complex power $f^{a}$ of a relative invariant $f$ of a prehomogeneous vector space, in (3.11). Next we describe the sheaves of holomorphic solutions of $D f^{a}$ and its Fourier transform, in (3.23). (See (2.3.1) for $D f^{\alpha}$.) In this section, we assume that a prehomogeneous vector space $(G, \rho, V)$, its dual $\left(G^{\vee}, \rho^{\vee}, V^{\vee}\right)$, relative invariants $f \in \mathbb{C}[V]$ and $f^{\vee} \in \mathbb{C}\left[V^{\vee}\right]$ are given. We use the notation given in the previous two sections. 
Lemma 3.耳. Let $u=\mathscr{F}\left(f^{\alpha}\right) \in \mathscr{F}\left(D f^{\alpha}\right)$ and consider the $D[s]$-module $D[s]\left(f^{\vee s} u\right)$. (Cf. (2.3.2).) In this $D[s]$-module, we have

$$
f(\partial)\left(f^{\vee} \circ f^{\vee s} \mathscr{F}\left(f^{\alpha}\right)\right)=(-1)^{d} b(\alpha-s-1) f^{\vee s} \mathscr{F}\left(f^{\alpha}\right) .
$$

Here $\partial=\operatorname{grad}=\left(\partial_{1}, \cdots, \partial_{n}\right)$.

Proof. For any natural number $l$,

$$
\begin{aligned}
& \left(f \circ f^{\vee}(\partial)-b(\alpha-l-1)\right) f^{\vee}(\partial)^{l} f^{\alpha} \\
& \quad=b(\alpha-1) \cdots b(\alpha-l) \cdot\left(f^{\circ} f^{\vee}(\partial)-b(\alpha-l-1)\right) f^{\alpha-l}=0 .
\end{aligned}
$$

Hence

$$
\left(f(\sqrt{-1} \partial) f^{\vee}(\sqrt{-1} y)-b(\alpha-l-1)\right) f^{\vee}(\sqrt{-1} y)^{l} \mathscr{F}\left(f^{\alpha}\right)=0 .
$$

Since $f$ and $f^{\vee}$ are homogeneous polynomials of degree $d$,

$$
\left(f(\partial) f^{\vee}-(-1)^{d} b(\alpha-l-1)\right) f^{\vee_{l}} \mathscr{F}\left(f^{\alpha}\right)=0 .
$$

As differential operators in $D[s]$, let

$$
\left(f^{\vee}\right)^{d-s}\left(f(\partial) f^{\vee}-(-1)^{d} b(\alpha-s-1)\right) f^{\vee s}=\sum_{j=0}^{d} s^{j} P_{j}
$$

with some $P_{j} \in D$. By (3.1.1), $P_{j} \mathscr{F}\left(f^{\alpha}\right)=0$, i.e.,

$$
\left.\left(f(\partial) f^{\vee}-(-1)^{d} b(\alpha-s-1)\right) f^{\vee s} \mathscr{F}^{\prime}\left(f^{\alpha}\right)\right)=0 .
$$

Lemma 3.2. If $\alpha \in A_{+}(b)$, then $\mathscr{I}\left(D f^{\alpha}\right)=\mathscr{F}\left(D f^{\alpha}\right)\left[f^{\vee-1}\right]$.

(See (1.6) for $b$, and (2.3.6) for $A_{+}$)

Proof. First, let us show that, if $u=P f^{\alpha} \in D f^{\alpha}$ satisfies $f^{\vee}(\partial) u=0$, then $u=0$. By the definition of $D f^{\alpha}$, there exists $Q(s) \in D[s]$ such that $f^{\vee}(\partial) P f^{s}=$ $(s-\alpha) Q(s) f^{s}$. Let $m$ be a sufficiently large integer. Then $Q(s) f^{\vee}(\partial)^{m}=$ $f^{\vee}(\partial) R(s)$ with some $R(s) \in D[s]$. Hence

$$
\begin{aligned}
& f^{\vee}(\partial) P f^{\vee}(\partial)^{m} f^{s+m}=b(s) b(s+1) \cdots b(s+m-1) f^{\vee}(\partial) P f^{s} \\
& \quad=b(s) \cdots b(s+m-1)(s-\alpha) Q(s) f^{s}=(s-\alpha) Q(s) f^{\vee}(\partial)^{m} f^{s+m} \\
& \quad=f^{\vee}(\partial)(s-\alpha) R(s) f^{s+m} .
\end{aligned}
$$

Let $N=D[s] f^{s}$. For $x \in \Omega,(x, \operatorname{grad} \log f(x)) \in W=\operatorname{ch} N$ by (2.4.1) and (2.4.6). Hence the principal symbol of $f^{\vee}(\partial)$ does not vanish identically on the characteristic variety of $N$. Thus, if $f^{v}(\partial) v=0$ for $v \in N$, then $D v$ is a holonomic submodule of $N$. Since $N$ does not have a holonomic submodule other than 0 by [13; Theorem (2.12) and Corollary (5.12)], $v=0$. Thus (3.2.1) implies 
$P f^{\vee}(\partial)^{m} f^{s+m}=(s-\alpha) R(s) f^{s+m}$ and

$$
P f^{\vee}(\partial)^{m} f^{s} \in(s-\alpha-m) N
$$

Define a $D$-module endomorphism $t^{\prime}$ of $N$ by

$$
t^{\prime}\left(T(s) f^{s}\right)=T(s-1) f^{\vee}(\partial) f^{s}\left(=b(s-1) T(s-1) f^{s-1}\right) .
$$

Then $s t^{\prime}-t^{\prime} s=t^{\prime}$, and $s$ induces a $D$-module endomorphism of $N / t^{\prime} N$. Since

$$
b(s-1) T(s) f^{s}=T(s)\left(f \circ f^{\vee}(\partial) f^{s}\right) \in t^{\prime} N,
$$

the minimal polynomial of $s \in \mathbb{E n d}\left(N / t^{\prime} N\right)$ is a divisor of $b(s-1)$. Let us show $\left(3.2 .2_{m}\right) \Rightarrow\left(3.2 .2_{m-1}\right)$ for $m \geq 1$. By our assumption, $b(\alpha+m-1) \neq 0$. Hence $s-\alpha-m \in \operatorname{End}\left(N / t^{\prime} N\right)$ is injective and

$$
\begin{aligned}
& t^{\prime}\left(P f^{\vee}(\partial)^{m-1} f^{s}\right)=P f^{\vee}(\partial)^{m} f^{s} \in(s-\alpha-m) N \cap t^{\prime} N=(s-\alpha-m) t^{\prime} N \\
& \quad=t^{\prime}(s-\alpha-m+1) N .
\end{aligned}
$$

Since $t^{\prime} \in$ End $N$ is injective, $P f^{\vee}(\partial)^{m-1} f^{s} \in(s-\alpha-m+1) N$. Thus inductively, we get $P f^{s} \in(s-\alpha) N$, i.e., $P f^{\alpha}=0$.

As we have shown above, $f^{\vee}(\partial)$ is not a zero divisor of $D f^{\alpha}$, and hence $f^{\vee}$ is not a zero divisor of $\mathscr{F}\left(D f^{\alpha}\right)$. Since $\alpha \in A_{+}(b(s))$ if and only if $0 \in$ $A_{-}(b(\alpha-s-1))$, we get the assertion from (3.1) and (2.3.11).

Lemma 3.3. $\operatorname{ch}\left(D f^{\alpha}\right) \cap\left(V \times \Omega^{\vee}\right)=\left(T O_{1}^{\vee}\right)^{\perp}$.

Proof. Let $\left(v, v^{\vee}\right)$ be an element of $\left(T O_{1}^{\vee}\right)^{\perp}$. By $(1.4,(2)), s^{-1} v^{\vee} \in O_{1}^{\vee}$ for any $s \in \mathbb{C}^{\times}$. Since

$$
T_{s^{-1} v^{\vee}} O_{1}^{\vee}=\operatorname{Lie}(G) \cdot\left(s^{-1} v^{\vee}\right)=s^{-1} \cdot \operatorname{Lie}(G) \cdot v^{\vee}=T_{v} \vee O_{1}^{\vee},
$$

we have $v \in T_{s^{-1} v \vee} O_{1}^{\vee}$, i.e., $\left(v, s^{-1} v^{\vee}\right) \in\left(T O_{1}^{\vee}\right)^{\perp}$. By $(1.18), \Phi\left(v, s^{-1} v^{\vee}\right)=v+$ $F^{\vee}\left(s^{-1} v^{\vee}\right)=v+s F^{\vee}\left(v^{\vee}\right)$ is an element of $F^{-1}\left(s^{-1} v^{\vee}\right)$, i.e., $F\left(v+s F^{\vee}\left(v^{\vee}\right)\right)=s^{-1} v^{\vee}$. Hence $\left(s, v+s F^{\vee}\left(v^{\vee}\right), v^{\vee}\right) \in \widetilde{W}$. By taking the limit $s \rightarrow 0$, we get $\left(0, v, v^{\vee}\right) \in \widetilde{W}_{0}$, and $\left(v, v^{\vee}\right) \in W_{0}=\operatorname{ch}\left(D f^{\alpha}\right)$ by $(2.4 .5)$ and $(2.4 .6,(2))$. Hence $\operatorname{ch}\left(D f^{\alpha}\right) \cap\left(V \times \Omega^{\vee}\right)$ $\supset\left(T O_{1}^{\vee}\right)^{\perp}$.

Conversely, let $\left(v, v^{\vee}\right)$ be an element of $\operatorname{ch}\left(D f^{\alpha}\right) \cap\left(V \times \Omega^{\vee}\right)$. By (2.4.5) and (2.4.6, (2)), $\left(0, v, v^{\vee}\right) \in \widetilde{W}_{0}$. By (2.4.2), there exists a sequence $\left(s_{k}, v_{k}, s_{k} F\left(v_{k}\right)\right)$ in $\mathbb{C}^{\times} \times \Omega \times V^{\vee}$ which converges to $\left(0, v, v^{\vee}\right)$. For any $A \in \operatorname{Lie}(G)$, $\left\langle v_{k} \mid A \cdot s_{k} F\left(v_{k}\right)\right\rangle=-s_{k}\left\langle A v_{k} \mid F\left(v_{k}\right)\right\rangle=-s_{k} \phi(A) \rightarrow 0$ by $(1.9,(1))$. On the other hand, the first member converges to $\left\langle v \mid A \circ v^{v}\right\rangle$. Hence $v$ is orthogonal to $\operatorname{Lie}(G) \circ v^{v}$. Since $s_{k} F\left(v_{k}\right)=F\left(s_{k}^{-1} v_{k}\right) \in O_{1}^{\vee}$ by (1.18), $v^{\vee} \in \bar{O}_{1}^{\vee}$. Since we are assuming $v^{\vee} \in$ $\Omega^{\vee}, v^{\vee} \in O_{1}^{\vee}$. Hence $\operatorname{Lie}(G) \circ v^{\vee}=T_{v^{\vee}} O_{1}^{\vee}$ and $v \in\left(T_{v^{\vee}} O_{1}^{\vee}\right)^{\perp}$. Thus we have 
proved that $\operatorname{ch}\left(D f^{\alpha}\right) \cap\left(V \times \Omega^{\vee}\right) \subset\left(T O_{1}^{\vee}\right)^{\perp}$.

Lemma 3.4. $\operatorname{ch}\left(\mathscr{I}\left(D f^{\alpha}\right)\right) \cap\left(V \times \Omega^{\vee}\right)=\left(T O_{1}^{\vee}\right)^{\perp}$.

Proof. We have

$$
\begin{aligned}
& \sum_{i=1}^{n} x_{i} \frac{\partial}{\partial x_{i}} f^{s}=d s f^{s}, \quad \sum_{i=1}^{n} x_{i} \frac{\partial}{\partial x_{i}} f^{\alpha}=d \alpha f^{\alpha}, \quad \text { and } \\
& d \alpha \mathscr{F}\left(f^{\alpha}\right)=\sum_{i=1}^{n} \sqrt{-1} \frac{\partial}{\partial y_{i}} \sqrt{-1} y_{i} \mathscr{F}\left(f^{\alpha}\right)=-\sum_{i=1}^{n} y_{i} \frac{\partial}{\partial y_{i}} \mathscr{F}\left(f^{\alpha}\right)-n \mathscr{F}\left(f^{\alpha}\right) .
\end{aligned}
$$

By (2.7.2) and (3.3), we have

$$
\operatorname{ch}\left(\mathscr{F}\left(D f^{\alpha}\right)\right) \cap\left(V \times \Omega^{\vee}\right)=-\sqrt{-1} \operatorname{ch}\left(D f^{\alpha}\right) \cap\left(V \times \Omega^{\vee}\right)=-\sqrt{-1}\left(T O_{1}^{\vee}\right)^{\perp} .
$$

Since it follows from (3.3.1) that $s\left(T O_{1}^{\vee}\right)^{\perp}=\left(T O_{1}^{\vee}\right)^{\perp}$ for any $s \in C^{\times}$, we get the assertion.

\subsection{D-modules $\mathbb{D} u_{\alpha}^{\prime}$ and $\boldsymbol{D} \boldsymbol{u}_{\alpha}^{\prime \prime}$}

By (1.9.1), we have

$$
\left(\sum_{i, j=1}^{n} a_{i j} x_{j} \frac{\partial}{\partial x_{i}}-s \phi(A)\right) f^{s}=0
$$

for any $A \in \operatorname{Lie}(G)$. (Here $\rho(A)=\left(a_{i j}\right)$.) By the definition of $f^{\alpha}$ (cf. (2.3.1)), and by the definition of the Fourier transformation (cf. (2.7.1)), we get

$$
\left(\sum_{i, j=1}^{n}\left(-a_{j i}\right) \frac{\partial}{\partial y_{i}} y_{j}-\alpha \phi(A)\right) \mathscr{L}\left(f^{\alpha}\right)=0 \text {. }
$$

Let $\phi_{0}(A)=\operatorname{Tr}(\rho(A))\left(=\sum_{i=1}^{n} a_{i i}\right)$, and $D u_{\alpha}^{\prime}$ be the $D\left(V^{\vee}\right)$-module defined by

$$
\left(\sum_{i, j=1}^{n}\left(-a_{j i}\right) y_{j} \frac{\partial}{\partial y_{i}}-\left(\alpha \phi+\phi_{0}\right)(A)\right) u_{\alpha}^{\prime}=0, \text { for } A \in \operatorname{Lie}(G) .
$$

Since $\mathscr{Z}\left(f^{\alpha}\right)$ solves this system of differential equations, we get a $D$-module homomorphism $\varphi: D u_{\alpha}^{\prime} \rightarrow D \mathscr{F}\left(f^{\alpha}\right)$ such that $\varphi\left(u_{\alpha}^{\prime}\right)=\mathscr{F}\left(f^{\alpha}\right)$. Let $I$ be the set of $a \in C\left[V^{\vee}\right]$ such that $a \equiv 0$ on $O_{1}^{\vee}$, and $D u_{\alpha}^{\prime \prime}$ the $D\left(V^{\vee}\right)$-module defined by (3.5.1) (with $u_{\alpha}^{\prime}$ replaced by $u_{\alpha}^{\prime \prime}$ ) and

$$
a u_{\alpha}^{\prime \prime}=0, \text { for } a \in I .
$$

Let $\psi: D u_{\alpha}^{\prime} \rightarrow D u_{\alpha}^{\prime \prime}$ be the natural morphism.

3.6. Let $J=\operatorname{ann}_{D}\left(\mathscr{F}\left(f^{\alpha}\right)\right), J^{\prime}=\operatorname{ann}_{D}\left(u_{\alpha}^{\prime}\right)$, and $\bar{J}$ be the ideal of $C\left[V \times V^{\vee}\right]$ generated by 


$$
\sigma_{1}\left(\sum_{i, j=1}^{n}\left(-a_{j i}\right) y_{j} \frac{\partial}{\partial y_{i}}-\left(\alpha \phi+\phi_{0}\right)(A)\right)=-\sum_{i, j=1}^{n} a_{j i} x_{i} y_{j}=-\langle y \mid A x\rangle 。
$$

(Here $\operatorname{ann}_{D}$ denotes the annihilator.) Then $\bar{J} \subset \operatorname{gr}\left(J^{\prime}\right) \subset \operatorname{gr}(J)$. See (2.1.1) for gr(-). For an ideal $a$ of $\mathbb{C}\left[V \times V^{\vee}\right]$, let $\mathbb{V}(\mathfrak{a})$ denote the algebraic set defined by $a$. Then by the proof of $(2.2 .5), \mathbb{V}\left(\operatorname{gr}\left(J^{\prime}\right)\right)=\operatorname{ch}\left(D u_{\alpha}^{\prime}\right)$ and $\mathbb{V}(\operatorname{gr}(J))=$ $\operatorname{ch}\left(D \mathscr{F}\left(f^{\infty}\right)\right)$. Hence, by (3.4),

$$
U \cap \nabla(\bar{J}) \supset U \cap \operatorname{ch}\left(D u_{a}^{\prime}\right) \supset U \cap \operatorname{ch}\left(D \mathscr{F}\left(f^{\infty}\right)\right)=U \cap\left(T O_{1}^{\vee}\right)^{\perp}
$$

for any open subset $U$ of $V \times \Omega^{\vee}$. Let $\mathbb{A}=\left(T O_{1}^{\vee}\right)^{\perp}$. By (1.18), $\Psi\left(O_{0}\right)$ is an open $G$-orbit in $\Lambda$. Let $p=\left(v, v^{\vee}\right)$ be a point in $\Psi\left(O_{0}\right)$. Then

$$
\begin{aligned}
& \operatorname{dim}\left\{\left(-A v, A v^{\vee}\right) \mid A \in \operatorname{Lie}(G)\right\}=\operatorname{dim}\left\{\left(A v, A v^{\vee}\right) \mid A \in \operatorname{Lie}(G)\right\} \\
& \quad=\operatorname{dim} G \circ\left(v, v^{\vee}\right)=\operatorname{dim} \Psi\left(O_{0}\right)=n .
\end{aligned}
$$

Since the differential at $p=\left(v, v^{\vee}\right)$ of the generators $-\langle y \mid A x\rangle$ of $\bar{J}$ are $-\langle A v \mid d y\rangle+\left\langle A v^{v} \mid d x\right\rangle$, since $\bar{J} \subset \operatorname{gr}(J)$, and since $\mathbb{V}(\operatorname{gr}(J))=\operatorname{ch}\left(D \mathscr{F}\left(f^{\alpha}\right)\right)$ coincides with $\mathbb{A}$ in a neighbourhood of $p$ by $(3.4), \operatorname{gr}(J)$ and $\operatorname{gr}\left(J^{\prime}\right)$ are also the defining ideals of $\Lambda$ in a neighbourhood of $p$.

Lemma 3.7. (1) With the notation as in (3.5) and (3.6), $E_{p} u_{\alpha}^{\prime}$ is a simple $E_{p}$-module for $p \in \Psi\left(O_{0}\right)$, i.e., it has no proper submodules.

(2) $m\left(\mathbb{1}, D u_{a}^{\prime}\right)=1$.

(Here and below, we write $E_{p} u_{\alpha}^{\prime}$ etc. for $E_{p} \otimes_{D} D u_{\alpha}^{\prime}$ etc.)

Proof. Assume first that $\operatorname{dim} O_{1}^{\vee}=n\left(=\operatorname{dim} V^{\vee}\right)$. Then $\Lambda \subset\{0\} \times V^{\vee}$, and $p=\left(0, v^{\vee}\right) \in \Psi\left(O_{0}\right)=O_{0}^{\vee}$. Let $A^{(k)} \in \operatorname{Lie}(G)(1 \leq k \leq n)$ be elements such that $\left\{A^{(1)} v^{\vee}, \ldots, A^{(n)} v^{\vee}\right\}$ are linearly independent, $\rho\left(A^{(k)}\right)=\left(a_{i j}^{(k)}\right)$ and $c_{i k}(y)=$ $-\sum_{j=1}^{n} a_{j i}^{(k)} y_{j}$. Then

$$
\left(\sum_{i=1}^{n} c_{i k}(y) \frac{\partial}{\partial y_{i}}-\left(\alpha \phi+\phi_{0}\right)\left(A^{(k)}\right)\right) u_{\alpha i}^{\prime}=0 \quad(1 \leq k \leq n),
$$

and $\left(c_{i k}\right)_{1 \leq i, k \leq n}$ is invertible in $M_{n}\left(A_{v} v\right)$, where $A_{v} v$ is the localization of $A=\mathbb{C}\left[\mathbb{V}^{\vee}\right]$ at $v^{\vee}$. Assume that $\sum_{k=1}^{n} c_{i k} \overline{c_{k j}}=\delta_{i j}$ and let $\sum_{k=1}^{n} \overline{c_{k i}}(y)\left(\alpha \phi+\phi_{0}\right)\left(A^{(k)}\right)=c_{i}(y)$. Then $\left(\partial_{i}-c_{i}(y)\right) u_{\alpha}^{\prime}=0(1 \leq i \leq n)$. For any $i$ and $j_{j} \partial_{i} \partial_{j} u_{\alpha}^{\prime}=\partial_{i}\left(c_{j} u_{\alpha}^{\prime}\right)=\partial_{i}\left(c_{j}\right) u_{\alpha}^{\prime}$ $+c_{j}\left(\partial_{i} u_{\alpha}^{\prime}\right)=\partial_{i}\left(c_{j}\right) u_{\alpha}^{\prime}+c_{i} c_{j} u_{\alpha}^{\prime}$. Hence $\left(\partial_{i}\left(c_{j}\right)-\partial_{j}\left(c_{i}\right)\right) u_{\alpha}^{\prime}=0$. Since $\operatorname{ch}\left(D u_{\alpha}^{\prime}\right) \supset$ $\operatorname{ch}\left(D \mathscr{F}\left(f^{\alpha}\right)\right) \supset \Lambda \supset O_{0}^{\vee}, \partial_{i}\left(c_{j}\right)=\partial_{j}\left(c_{i}\right)$. Hence we can find $c \in \tilde{A}_{v} v$ such that $c_{i}=\partial_{i}(c)$. (See (2.1.3) for $\left.\tilde{\mathbb{A}}_{v} v\right)$. Then $\partial_{\imath}\left(e^{-c} u_{\alpha}^{\prime}\right)=0(1 \leq i \leq n)$. Hence $\mathbb{E}_{p} u_{\alpha}^{\prime}$ is isomorphic to a quotient of the $E_{p}$-module defined by $\partial_{1} u=\cdots=\partial_{n} u=0$. (In the present case, $E_{p}=\tilde{D}_{v^{v}}$ ). Since the latter is a simple $E_{p}$-module and $p \in O_{0}^{\vee}$ 
$\subset \operatorname{ch}\left(D u_{\alpha}^{\prime}\right), E_{p} u_{\alpha}^{\prime}$ is non-zero and is a simple $E_{p}$-module.

Let us consider the case where $\operatorname{dim} O_{1}^{\vee}<n$. Then $p \notin\{0\} \times V^{\vee}$. (In fact, if $p \in\{0\} \times V^{\vee}, \Psi\left(O_{0}\right)$ should be contained in $\left.\Lambda \cap\left(\{0\} \times V^{\vee}\right)=\{0\} \times O_{1}^{\vee}\right)$. By (3.6), $E_{p} u_{\alpha}^{\prime}$ is generated by a non-degenerate element $u_{\alpha}^{\prime}$ [26; Chap. II, Def. 4.1.1]. Let $p^{\prime}=\left(x_{1}, \cdots, x_{n} ; y_{1}, \cdots, y_{n}\right)=(0, \cdots, 0 ; 1,0, \cdots, 0)$ and, for $\beta \in \mathbb{C}$, $M(\beta)=E_{p^{\prime}} u$ be the $E_{p^{\prime}}$-module defined by $\left(x_{1} \partial_{1}-\beta\right) u=\partial_{2} u=\cdots=\partial_{n} u=0$. Then by a suitable quantized contact transformation, $E_{p} u_{\alpha}^{\prime}$ can be transformed into $M(\beta)$ for some $\beta$ [25; Theorem 8.2, (2)]. Since $M(\beta)$ is a simple $E_{p^{\prime \prime}}$ module, $E_{p} u_{\alpha}^{\prime}$ is also a simple $E_{p}$-module.

The second assertion is proved in the same way. (In fact, (1) follows from (2). In order to see the invariance of the multiplicity with respect to quantized contact transformations, it is enough to note that our definition is equivalent to $[15 ; 2.6 .1]$.)

Remark 3.7.1. The above lemma holds for any good Lagrangian variety $\Lambda$ and its generic point $p$. Cf. [25].

Remark 3.8. By using the notation of [25; §8] and [26; Chap. II, §4.2], the second assertion of [25; Theorem 8.2] is deduced from [26; Chap. II, Theorem 4.2.5] as follows. Let $p$ be a point which does not belong to the zero section of $T^{*} X$, and $M$ a holonomic system generated by a non-degenerate section $u$ in a neighbourhood of $p$. Since every canonical transformation can be 'quantized' locally $[15 ; 2.4 .16]$, we can 'quantize' Theorem 8.1 of [25]. Hence we may assume from the beginning that $M$ is supported by $\left\{(z ; \xi) \in T^{*} X \mid z_{1}=\xi_{2}=\xi_{3}=\right.$ $\left.\cdots=\xi_{n}=0\right\}$, and $p=(z ; \xi)$ with $\xi_{1} \neq 0$. Let $\alpha=-\operatorname{ord}(u)-\frac{1}{2}$, and $M^{\prime}$ be the system $\left(z_{1} D_{1}-\alpha\right) v=D_{2} v=\cdots=D_{n} v=0$. Then $\operatorname{ord}(v)=-\alpha-\frac{1}{2}=\operatorname{ord}(u)$. Hence $M$ is isomorphic to $M^{\prime}$ in a neighbourhood of $p$ by [26; Chap. II, Theorem 4.2.5].

Lemma 3.9. Let $p$ be an element of $\Psi\left(O_{0}\right)$. The morphisms $\varphi$ and $\psi$ in (3.5) induce isomorphisms $\varphi: E_{p} u_{\alpha}^{\prime} \rightarrow E_{p} \mathscr{F}^{\prime}\left(f^{\alpha}\right)$ and $\psi: E_{p} u_{\alpha}^{\prime} \rightarrow E_{p} u_{\alpha}^{\prime \prime}$.

Proof. Since $E_{p} u_{\alpha}^{\prime}$ is a simple $E_{p}$-module by (3.7), and since $\varphi$ and $\psi$ are surjective homomorphisms, it is enough to prove that

$$
\begin{aligned}
E_{p} u_{\alpha}^{\prime \prime} \neq 0 \text { and } & \\
E_{p} \mathcal{F}\left(f^{\alpha}\right) & \neq 0 .
\end{aligned}
$$

(3.9.2) is already proved in (3.4). Cf. (2.2.5). Hence it is enough to prove that 


$$
\operatorname{ch}\left(D\left(\Omega^{\vee}\right) u_{\alpha}^{\prime \prime}\right)=\left(T O_{1}^{\vee}\right)^{\perp} .
$$

Since the algebraic subset of $V \times \Omega^{\vee}$ defined by the principal symbols of the equations (3.5.1) and (3.5.2) is $\left(T O_{1}^{\vee}\right)^{\perp}$,

$$
\operatorname{ch}\left(D\left(\Omega^{\vee}\right) u_{\alpha^{\prime \prime}}^{\prime \prime}\right) \subset\left(T O_{1}^{\vee}\right)^{\perp} .
$$

What is necessary for our purpose is the inverse inclusion, which will be proved in the course of a detailed study of $D u_{a}^{\prime \prime}$. A proof of (3.9.3) will be given in (3.18). By $(3.7,(2)), m\left(\left(T O_{1}^{\vee}\right)^{\perp}, D u_{x}^{\prime}\right)=1$. Hence it follows from (3.9.3) that

$$
\operatorname{cln}\left(D\left(\Omega^{\vee}\right) u_{a}^{\prime \prime}\right)=\left(T O_{1}^{\vee}\right)^{\perp}
$$

Together with (2.2.6), (3.9.4) implies that $D\left(\Omega^{\vee}\right) u_{\alpha}^{\prime \prime}$ is a simple $D\left(\Omega^{\vee}\right)$-module.

Before proving (3.9.3), let us prove our first main result of this section assuming (3.9).

Lemma 3.10。 Let I be as in (3.5). Then $a \mathscr{F}\left(f^{a}\right)=0$ for any $a \in \mathbb{I}$.

Proof. For any $a \in \mathbb{I}, a u_{\alpha}^{\prime \prime}=0$. By (3.9), $a \mathscr{F}\left(f^{\alpha}\right)=0$ as an element of $E_{p} \mathscr{F}\left(f^{\alpha}\right)$ for any $p \in \Psi\left(O_{0}\right)$. By (2.2.5),

$$
\Psi\left(O_{0}\right) \cap \operatorname{ch}\left(\operatorname{DaF}\left(f^{\alpha}\right)\right)=\phi .
$$

By (3.4),

$$
\begin{aligned}
\operatorname{ch}\left(\operatorname{Da\mathscr {F}}\left(f^{\alpha}\right)\right) \cap\left(V \times \Omega^{\vee}\right) & =\operatorname{ch}\left(D a \mathscr{F}\left(f^{\alpha}\right)\right) \cap \operatorname{ch}\left(D \mathscr{F}\left(f^{\alpha}\right)\right) \cap\left(V \times \Omega^{\vee}\right) \\
& =\operatorname{ch}\left(D a \mathscr{F}\left(f^{\alpha}\right)\right) \cap \Lambda \subset \Lambda-\Psi\left(O_{0}\right) .
\end{aligned}
$$

Hence for any irreducible component $\Lambda^{\prime}$ of $\operatorname{ch}\left(D a \mathscr{F}\left(f^{\infty}\right)\right)$ such that $\Lambda^{\prime} \cap(V \times$ $\left.\Omega^{\vee}\right) \neq \phi, \Lambda^{\prime} \cap\left(V \times \Omega^{\vee}\right) \subset \Lambda-\Psi\left(O_{0}\right)$. Hence $\operatorname{dim} \Lambda^{\prime}<\operatorname{dim} \Lambda=n$. Since a characteristic variety is known to be involutive [26; Chap. II, Theorem 5.3.2], $\operatorname{dim} \Lambda^{\prime}$ is at least $n$. Hence such an irreducible component does not exist, i.e.,

$$
\operatorname{ch}\left(\operatorname{Da\mathscr {F}}\left(f^{\alpha}\right)\right) \cap\left(V \times \Omega^{\vee}\right)=\phi .
$$

By (2.2.7), $\operatorname{supp}\left(a \mathscr{F}^{\prime}\left(f^{\alpha}\right)\right) \cap \Omega^{\vee}=\phi, \quad$ and $a \mathscr{F}\left(f^{\alpha}\right)=0$ as an element of $\mathscr{I}\left(D f^{\alpha}\right)\left[f^{\vee-1}\right]$. By (3.2), if $\alpha \in A_{+}(b), a \mathscr{F}\left(f^{\alpha}\right)=0$ as an element of $\mathscr{F}\left(D f^{\alpha}\right)$. Hence, for $\alpha \in A_{+}(b), a(\partial) f^{s} \in(s-\alpha) D[s] f^{s}$. Recall that $f(x)^{s}$ is a holomorphic function of $(s, x) \in \mathbb{C} \times B$ (cf. (2.3.1)). We can consider its restriction to $\{\alpha\} \times$ $B$, and $\left.a(\partial) f^{s}\right|_{\{\alpha\} \times B} \equiv 0$ for $\alpha \in A_{+}(b)$. Hence

$$
a(\partial) f^{s}=0 \text { for } a \in I .
$$

By the definition of $f^{a}$ and $\mathscr{F}$, we get the assertion. 
Theorem 3.11. Let

$$
\begin{aligned}
& A_{+}=\{\alpha \in C \mid b(\alpha+j) \neq 0 \quad \text { for } j=0,1,2, \cdots\} \quad \text { and } \\
& A_{-}=\{\alpha \in C \mid b(\alpha-j) \neq 0 \quad \text { for } j=1,2, \cdots\} .
\end{aligned}
$$

(1) $D f^{\alpha}=\left(D f^{\alpha}\right)\left[f^{-1}\right]$, if $\alpha \in A_{-}$.

(2) $D f^{\alpha}=\left(\left(D f^{-\alpha}\right)\left[f^{-1}\right]\right)^{*}$, if $\alpha \in A_{+}$.

(3) $\mathscr{F}\left(D f^{\alpha}\right)=\mathscr{F}\left(D f^{\alpha}\right)\left[f^{v-1}\right]$, if $\alpha \in A_{+}$.

(4) $\mathscr{I}\left(D f^{\alpha}\right)=\left(\mathscr{F}\left(D f^{-\alpha}\right)\left[f^{\vee-1}\right]\right)^{*}$, if $\alpha \in A_{\text {-. }}$.

(5) Let $D u_{\alpha}^{\prime \prime}$ be the D-module defined by

$$
\left(\sum_{i, j=1}^{n}\left(-a_{j i}\right) y_{j} \frac{\partial}{\partial y_{i}}-\left(\alpha \phi+\phi_{0}\right)(A)\right) u_{\alpha}^{\prime \prime}=0 \quad \text { for } \quad A \in \operatorname{Lie}(G),
$$

and

$$
a u_{\alpha}^{\prime \prime}=0 \text { for } a \in I .
$$

(Here $\rho(A)=\left(a_{i j}\right), \phi_{0}=\operatorname{Tr}(\rho(A))$ and $I$ is the defining ideal of $\bar{O}_{1}^{\vee}$.) Then for any $\alpha \in \boldsymbol{C}$ and for any integer $k$,

$$
\mathscr{F}\left(D f^{\alpha}\right)\left[f^{\vee-1}\right]=\left(D u_{\alpha+k}^{\prime \prime}\right)\left[f^{\vee-1}\right] .
$$

(See (1.6) for $b(s),(2.3 .1)$ for $D f^{\alpha},(2.6 .3)$ for $*$, and (2.7.1) for $\mathscr{F}$.)

Proof. (1) follows from (2.3.8). (Cf. the remark at the end of (2.3.2).) If $\alpha \in A_{+}, D f^{\alpha+k}=D f^{\alpha}$ for any non-negative integer $k$ by (2.3.9). If $k$ is sufficiently large, $-\alpha-k \in A_{-}$and $D f^{\alpha}=D f^{\alpha+k}=\left(D f^{-\alpha-k}\right)^{*}=\left(\left(D f^{-\alpha}\right)\left[f^{-1}\right]\right)^{*}$ by (2.6.4, (3)). Cf. (2.5.10). (3) is already proved in (3.2). Let us prove (5). By (3.10), $\varphi: D u_{\alpha}^{\prime} \rightarrow D \mathscr{F}\left(f^{\alpha}\right)$ induces a surjective morphism $\bar{\varphi}: D u_{\alpha}^{\prime \prime} \rightarrow D \mathscr{F}\left(f^{\alpha}\right)$ such that $\bar{\varphi}\left(u_{\alpha}^{\prime \prime}\right)=\mathscr{F}\left(f^{\alpha}\right)$. By (3.5.1) and (3.5.2), the characteristic variety of $D u_{\alpha}^{\prime \prime}$ is contained in the variety defined by $\langle y|A| x\rangle=0(A \in \operatorname{Lie}(G))$, and $a(y)$ $=0(a \in I)$. Hence

$$
\operatorname{ch}\left(D u_{\alpha}^{\prime \prime}\right) \cap\left(V \times \Omega^{\vee}\right) \subset\left(T O_{1}^{\vee}\right)^{\perp}=\Lambda .
$$

On the other hand $\operatorname{ch}\left(D \mathscr{F}\left(f^{\alpha}\right)\right) \cap\left(V \times \Omega^{\vee}\right)=\Lambda$ by (3.4), and $\bar{\varphi}$ induces an isomorphism $E_{p} u_{\alpha}^{\prime \prime} \rightarrow E_{p} \mathscr{F}\left(f^{\alpha}\right),\left(p \in \Psi\left(O_{0}\right)\right)$ by (3.9). Hence $\operatorname{ch}(\operatorname{ker} \bar{\varphi}) \cap\left(V \times \Omega^{\vee}\right)$ is contained in $A-\Psi\left(O_{0}\right)$. Since a characteristic variety is involutive, $\operatorname{ch}(\operatorname{ker} \bar{\varphi}) \cap$ $\left(V \times \Omega^{\vee}\right)=\phi . \quad$ By $(2.2 .7), \operatorname{supp}(\operatorname{ker} \bar{\varphi}) \subset V^{\vee}-\Omega^{\vee}=f^{\vee-1}(0)$. Hence $(\operatorname{ker} \bar{\varphi})\left[f^{\vee-1}\right]$ $=0$ and $\left(D u_{\alpha}^{\prime \prime}\right)\left[f^{\vee-1}\right] \simeq \mathscr{F}\left(D f^{\alpha}\right)\left[f^{\vee-1}\right]$. Since the morphism $D u_{\alpha-1}^{\prime \prime} \rightarrow D u_{\alpha}^{\prime \prime}$ defined by $u_{\alpha-1}^{\prime \prime} \rightarrow f^{\vee} u_{\alpha}^{\prime \prime}$ induces an isomorphism $\left(D u_{\alpha-1}^{\prime \prime}\right)\left[f^{\vee-1}\right] \simeq\left(D u_{\alpha}^{\prime \prime}\right)\left[f^{\vee-1}\right]$, we get the assertion. Let us prove (4). Assume that $\alpha \in A_{-}$. For a sufficiently large integer $k,-\alpha+k \in A_{+}$. Hence 


$$
\begin{aligned}
\left(\mathscr{F}\left(D f^{-\alpha}\right)\left[f^{\vee-1}\right]\right)^{*} & =\left(\mathscr{F}\left(D f^{-\alpha+k}\right)\left[f^{\vee-1}\right]\right)^{*}, \quad \text { by }(5), \\
& =\mathscr{F}\left(D f^{-\alpha+k}\right)^{*}, \quad \text { by }(3), \\
& =\mathscr{F}\left(\left(D f^{-\alpha+k}\right)^{*}\right) \\
& =\mathscr{F}\left(\left(D f^{\alpha-k}\right)\left[f^{-1}\right]\right), \quad \text { by the dual of }(2), \\
& =\mathscr{F}\left(D f^{\alpha}\right) .
\end{aligned}
$$

Thus we get (4).

3.12. Let $j: \Omega \rightarrow V, j^{\vee}: \Omega^{\vee} \rightarrow V^{\vee}, i: O_{1} \rightarrow \Omega$ and $i^{\vee}: O_{1}^{\vee} \rightarrow \Omega^{\vee}$ be the inclusion mappings. Let $\operatorname{dim} O_{1}^{\vee}=m, v^{\vee} \in O_{1}^{\vee},\left\{y_{1}^{\prime}, \cdots, y_{m}^{\prime}\right\}$ be a local coordinate system of $O_{1}^{v}$ at $v^{v}$, and

$$
x_{k}^{\prime}=F^{*} y_{k}^{\prime}
$$

Then $\left\{x_{1}^{\prime}, \cdots, x_{m}^{\prime}\right\}$ gives a local coordinate system of $O_{1}$ at $v=F^{\vee}\left(v^{\vee}\right)$. Let $\frac{\partial}{\partial x_{k}^{\prime}}$ and $\frac{\partial}{\partial y_{k}^{\prime}}$ be the tangent vector field determined by these coordinate functions. We sometimes write $\partial_{k}^{\prime}$ for $\frac{\partial}{\partial x_{k}^{\prime}}$ or $\frac{\partial}{\partial y_{k}^{\prime}}$ if there is no fear of confusion. We denote by $\partial_{k, p}^{\prime}$ the 'value' of $\partial_{k}^{\prime}$ at a point $p$.

Denote by $F_{*, v}^{\vee}$ the linear mapping $T_{v} \vee O_{1}^{\vee} \rightarrow T_{v} O_{1}$ induced by $F^{\vee}$. Define a bilinear form $B_{v}$ on $T_{v} v O_{1}^{\vee}$ by

$$
B_{v^{\vee}}(p, q)=\left\langle F_{*, v}^{\vee}(p) \mid q\right\rangle
$$

for $p, q \in T_{v} v O_{1}^{\vee}$. Here we regard $T_{v} O_{1}$ and $T_{v} v O_{1}^{\vee}$ as subspaces of $V$ and $V^{v}$. Since this bilinear form is nothing but the one defined in (1.16), $B_{v^{v}}$ is $G_{v^{v}}$ invariant, non-degenerate and symmetric. Let

$$
\omega^{\vee 2}\left(v^{\vee}\right)=\operatorname{det}\left(B_{v} v\left(\partial_{k, v}^{\prime}, \partial_{l, v}^{\prime}\right)\right)_{1 \leq k, l \leq m} \cdot\left(d y_{1}^{\prime} \wedge \cdots \wedge d y_{m}^{\prime}\right)^{\otimes 2}
$$

and $\omega^{\vee 2}$ be the section of $\left(\Omega_{O_{1}^{\vee}}\right)^{\otimes 2}$ defined by $v^{\vee} \rightarrow \omega^{\vee 2}\left(v^{\vee}\right)$. (See (2.8.1) for $\Omega_{X}$.) Note that $\omega^{\mathrm{v} 2}$ does not vanish at any point of $O_{1}^{\mathrm{v}}$.

Lemma 3.13. $\omega^{\mathrm{v} 2}$ is G-invariant and does not depend on the choice of a local coordinate system $\left\{y_{1}^{\prime}, \cdots, y_{m}^{\prime}\right\}$.

Proof. For $g \in G$, let

$$
g_{*}^{-1}\left(\partial_{j, g v}^{\prime}\right)=\sum_{i=1}^{m} c_{i j}\left(v^{\vee}\right) \partial_{i, v^{\vee}}^{\prime} .
$$

Then $\left(g^{-1}\right)^{*} d y_{i}^{\prime}=\sum_{j=1}^{m} c_{i j} d y_{j}^{\prime}$, and, hence, 


$$
g^{*}\left(d y_{1}^{\prime} \wedge \cdots \wedge d y_{m}^{\prime}\right)^{\otimes 2}=\operatorname{det}\left(c_{i j}\right)^{-2}\left(d y_{1}^{\prime} \wedge \cdots \wedge d y_{m}^{\prime}\right)^{\otimes 2}
$$

Since

$$
\begin{aligned}
& T_{g v \vee} O_{1}^{\vee} \stackrel{F_{*, g v \vee}^{\vee}}{\longrightarrow} T_{g v} O_{1} \\
& g_{*}^{-1} \downarrow \\
& T_{v \vee} O_{1}^{\vee} \stackrel{F_{*, v \vee}^{\vee}}{\longrightarrow} T_{v} O_{1}
\end{aligned}
$$

is commutative, we have

$$
\begin{aligned}
& B_{g v v}\left(\partial_{i, g v v}^{\prime}, \partial_{j, g v v}^{\prime}\right)=\left\langle F_{*, g v v}^{\vee}\left(\partial_{i, g v v}^{\prime}\right) \mid \partial_{j, g v v}^{\prime}\right\rangle \\
& =\left\langle g_{*} \cdot F_{*, v v}^{\vee} \cdot g_{*}^{-1}\left(\partial_{i, g v v}^{\prime}\right) \mid \partial_{j, g v}^{\prime}\right\rangle \\
& =\left\langle F_{*, v}^{\vee} \cdot g_{*}^{-1}\left(\partial_{i, g v}^{\prime}\right) \mid g_{*}^{-1}\left(\partial_{j, g v}^{\prime} v\right)\right\rangle \\
& =\sum_{i^{\prime}, j^{\prime}=1}^{m} c_{i^{\prime} i}\left(v^{\vee}\right) c_{j^{\prime} j}\left(v^{\vee}\right)\left\langle F_{*, v \vee}^{\vee}\left(\partial_{i^{\prime}, v^{v}}^{\prime}\right) \mid \partial_{j^{\prime}, v^{\vee}}^{\prime}\right\rangle \\
& =\sum_{i^{\prime}, j^{\prime}=1}^{m} c_{i^{\prime} i}\left(v^{v}\right) c_{j^{\prime} j}\left(v^{\vee}\right) B_{v^{v}}\left(\partial_{i^{\prime}, v v}^{\prime}, \partial_{j^{\prime}, v v}^{\prime}\right)
\end{aligned}
$$

and, hence,

$$
\operatorname{det}\left(B_{g v v}\left(\partial_{i, g v v}^{\prime}, \partial_{j, g v}^{\prime}\right)\right)=\operatorname{det}\left(c_{i j}\right)^{2} \operatorname{det}\left(B_{v v}\left(\partial_{i, v v}^{\prime}, \partial_{j, v}^{\prime}\right)\right) .
$$

By (3.13.1) and (3.13.2), we get the $G$-invariance. The independence of the choice of a local coordinate system is obvious.

3.14. Let $\omega^{\vee}$ be a local single-valued $m$-form on $O_{1}^{\vee}$ such that $\omega^{\vee} \otimes \omega^{\vee}=$ $\omega^{\vee 2}$. Although $\omega^{\vee}$ is not necessarily single-valued on the whole of $O_{1}^{\vee}$, but there is a canonical two-fold covering $\pi^{\vee}: \tilde{O}_{1}^{\vee} \rightarrow O_{1}^{\vee}$ such that $\widetilde{\omega}^{\vee}:=\pi^{*} \omega^{\vee}$ becomes globally single-valued. This two-fold covering is constructed as follows: Let $\left(U^{(\nu)} ; z_{1}^{(\nu)}, \cdots, z_{m}^{(\nu)}\right)$ be the local coordinate systems of $O_{1}^{\vee}$. If $\omega^{\vee 2}$ is given by

$$
\omega^{\mathrm{v} 2}=a^{(\nu)}\left(z_{1}^{(\nu)}, \cdots, z_{m}^{(\nu)}\right) \cdot\left(d z_{1}^{(\nu)} \wedge \cdots \wedge z_{m}^{(\nu)}\right)^{\otimes 2},
$$

we can define a two-fold (unramified) covering $\widetilde{U}^{(\nu)}$ of $U^{(\nu)}$ by

$$
\begin{array}{r}
\tilde{U}^{(\nu)}=\left\{\left(z_{0}^{(\nu)}, z_{1}^{(\nu)}, \cdots, z_{m}^{(\nu)}\right) \mid\left(z_{0}^{(\nu)}\right)^{2}=a^{(\nu)}\left(z_{1}^{(\nu)}, \cdots, z_{m}^{(\nu)}\right),\right. \\
\left.z_{0}^{(\nu)} \in C,\left(z_{1}^{(\nu)}, \cdots, z_{m}^{(\nu)}\right) \in U^{(\nu)}\right\} .
\end{array}
$$

Identify $\left(z_{0}^{(\mu)}, \cdots, z_{m}^{(\mu)}\right) \in \widetilde{U}^{(\mu)}$ with $\left(z_{0}^{(\nu)}, \cdots, z_{m}^{(\nu)}\right) \in \widetilde{U}^{(\nu)}$ if $\left(z_{1}^{(\mu)}, \cdots, z_{m}^{(\mu)}\right) \in U^{(\mu)}$ and $\left(z_{1}^{(\nu)}, \cdots, z_{m}^{(\nu)}\right) \in U^{(\nu)}$ correspond to the same point of $O_{1}^{v}$ and

$$
\frac{z_{0}^{(\mu)}}{z_{0}^{(\nu)}} \cdot \frac{d z_{1}^{(\mu)} \wedge \cdots \wedge d z_{m}^{(\mu)}}{d z_{1}^{(\nu)} \wedge \cdots \wedge d z_{m}^{(\nu)}}=1
$$


Patching together $U^{(v)}$ 's in this way, we get a two-fold (unramified) covering $\tilde{O}_{1}^{\vee}$ of $O_{1}^{v}$.

Define a two-fold covering $\pi: \widetilde{\Omega} \rightarrow \Omega$ as a pull-back of $\pi^{\vee}: \tilde{O}_{1}^{\vee} \rightarrow O_{1}^{\vee}$ by $F: \Omega \rightarrow O_{1}^{\vee}$, i.e., $\tilde{\Omega}=\tilde{O}_{1}^{\vee} \times_{O_{1}^{\vee}} \Omega$. We define $B_{v}, \omega, \widetilde{\omega}, \pi: \tilde{O}_{1} \rightarrow O_{1}$ and $\pi^{\vee}: \tilde{\Omega}^{\vee} \rightarrow \Omega^{\vee}$ in the same way as above.

Lemma 3.15. The isomorphism $F: O_{1} \rightarrow O_{1}^{\vee}$ can be lifted to an isomorphism $\tilde{F}: \tilde{O}_{1} \rightarrow \tilde{O}_{1}^{\vee}$, i.e., there is a commutative diagram

$$
\begin{gathered}
\tilde{O}_{1} \stackrel{\widetilde{F}}{\longrightarrow} \tilde{O}_{1}^{\vee} \\
\pi \downarrow \pi^{\vee} \downarrow \\
O_{1} \stackrel{F}{\longrightarrow} O_{1}^{\vee} .
\end{gathered}
$$

Proof. It is enough to prove that

$$
F^{*} \omega^{\mathrm{\vee} 2}=\omega^{2}
$$

By (3.12.1),

$$
F^{*} d y_{k}^{\prime}=d x_{k}^{\prime} \quad \text { and } \quad F_{*}\left(\frac{\partial}{\partial x_{k}^{\prime}}\right)=\frac{\partial}{\partial y_{k}^{\prime}}
$$

Hence for $v \in O_{1}$ and $F(v)=v^{v} \in O_{1}^{v}$,

$$
\begin{aligned}
B_{v v}\left(\partial_{k, v v}^{\prime}, \partial_{l, v v}^{\prime}\right) & =\left\langle F_{*, v v}^{\vee}\left(\partial_{k, v}^{\prime}\right) \mid \partial_{l, v v}^{\prime}\right\rangle \\
& =\left\langle F_{*, v v}^{\vee} F_{*, v}\left(\partial_{k, v}^{\prime}\right) \mid F_{*, v}\left(\partial_{l, v}^{\prime}\right)\right\rangle \\
& =\left\langle F_{*, v} F_{*, v}^{\vee} F_{*, v}\left(\partial_{k, v}^{\prime}\right) \mid \partial_{l, v}^{\prime}\right\rangle .
\end{aligned}
$$

(Recall that $F_{*, v}$ corresponds to the symmetric matrix $\left(\frac{\partial^{2} \log f}{\partial x_{i} \partial x_{j}}(v)\right)$.) Since $F_{v \vee}^{\vee} \cdot F_{v}$ is the identity mapping,

$$
B_{F(v)}\left(\partial_{k, v v}^{\prime}, \partial_{l, v v}^{\prime}\right)=\left\langle F_{*, v}\left(\partial_{k, v}^{\prime}\right) \mid \partial_{l, v}^{\prime}\right\rangle=B_{v}\left(\partial_{k, v}^{\prime}, \partial_{l, v}^{\prime}\right) .
$$

By this equality, together with (3.15.2), we get the assertion.

3.16. By (3.15), we may regard $\tilde{O}_{1}=\tilde{O}_{1}^{\vee} \times{ }_{o_{1}^{\vee}} O_{1}$. Let $\tilde{i}: \tilde{O}_{1} \rightarrow \tilde{\Omega}\left(=\tilde{O}_{1}^{\vee} \times{ }_{o_{1}^{\vee} \Omega}\right)$ be the injection induced by $i: O_{1} \rightarrow \Omega$. Define $\tilde{i}^{\vee}: \tilde{O}_{1}^{\vee} \rightarrow \tilde{\Omega}^{\vee}$ in the same way. Let $\mathcal{I} \subset \mathcal{O}_{\tilde{\Omega}^{v}}$ be the defining ideal of $\tilde{O}_{1}^{v}$. Recall that $\mathscr{D}_{\tilde{o}_{1}^{v} \rightarrow \tilde{\Omega}^{v}}=\mathcal{O}_{\tilde{o}_{1}^{v}} \otimes \tilde{i}^{v-1} \mathscr{D}_{\tilde{\Omega}^{v}}$ $=\left(\mathscr{D}_{\tilde{\Omega}^{v}} / \mathcal{G}_{\mathscr{D}_{\Omega^{v}}}\right) \mid \tilde{o}_{1}^{v}$, where, in the second member, the tensor product is taken over $\tilde{i}^{\vee-1} \mathcal{O}_{\widetilde{\Omega}^{\vee}}(\mathrm{cf} .(2.1 .4))$.

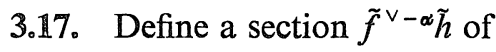




$$
\int_{\tilde{i}^{\vee}} \mathscr{D}_{\tilde{o}_{1}^{v}} \tilde{f}^{\vee-\alpha}:=\tilde{i}_{*}^{v}\left(\tilde{f}^{\vee-\alpha} \Omega_{\tilde{o}_{1}^{\vee}} \otimes \mathscr{D}_{\tilde{o}_{1}^{v} \rightarrow \tilde{\Omega}^{v}}\right) \otimes \Omega_{\tilde{\Omega}^{v}}^{-1}
$$

by

$$
\tilde{f}^{\vee-\alpha} \tilde{h}=\frac{\tilde{f}^{\vee-\alpha} \tilde{\omega}^{\vee} \otimes 1 \tilde{o}_{1}^{\vee} \rightarrow \tilde{\Omega}^{\vee}}{\pi^{\vee *}\left(d y_{1} \wedge \cdots \wedge d y_{n}\right)} .
$$

(See (2.8.1) for $\Omega_{X}$.) Here $\tilde{f}^{\vee}=\pi^{\vee} * f^{\vee}$. Let us show that there exists a nonzero homomorphism

$$
\pi^{\vee-1} \mathcal{D}_{\Omega^{\vee}} u_{\alpha}^{\prime} \rightarrow \int_{\tilde{i}^{\vee}} \mathcal{D}_{\tilde{o}_{1}^{\vee}} \tilde{f}^{\vee-\alpha}
$$

such that $u_{\alpha}^{\prime} \rightarrow \tilde{f}^{\vee-\alpha} \tilde{h}$. Since $\tilde{f}^{\vee-\alpha} \tilde{h} \neq 0$ by $(2.1 .5)$, it is enough to prove that $\tilde{f}^{\vee-\alpha} \tilde{h}$ solves (3.5.1) locally, i.e.,

$$
\left(f^{\vee-\alpha} \omega^{\vee} \otimes 1_{O_{1}^{\vee} \rightarrow \Omega^{\vee}}\right)\left(\sum_{i, j=1}^{n} a_{j i} \frac{\partial}{\partial y_{i}} y_{j}-\left(\alpha \phi+\phi_{0}\right)(A)\right)=0 .
$$

Since $\tilde{O}_{1}^{\vee}$ is locally isomorphic to $O_{1}^{\vee}$, we may identify $\tilde{f}^{\vee-\infty} \tilde{h}$ with the local section $f^{\vee-\infty} h$ of $\int_{i^{\vee}} \mathscr{D}_{O_{1}^{\vee}}^{a n} f^{\vee-\infty}$ defined by

$$
f^{\vee-\alpha} h=\frac{f^{\vee-\alpha} \omega^{\vee} \otimes 1_{O_{1}^{\vee} \rightarrow \Omega^{\vee}}}{d y_{1} \wedge \cdots \wedge d y_{n}}
$$

Let $\left\{z_{1}, \cdots, z_{n}\right\}$ be a local coordinate system of $\Omega^{\vee}$ such that $\left\{z_{m+1}=\cdots=z_{n}=0\right\}$ $=O_{1}^{\vee}$, and $z_{i}^{\prime}=\left.z_{i}\right|_{o_{1}^{\vee}}(1 \leq i \leq m)$. Then $\left\{z_{1}^{\prime}, \cdots, z_{m}^{\prime}\right\}$ gives a local coordinate system of $O_{1}^{\vee}$. Let

$$
f^{\vee-\infty} \omega^{\vee}=e\left(z_{1}^{\prime}, \cdots, z_{m}^{\prime}\right) d z_{1}^{\prime} \wedge \cdots \wedge d z_{m}^{\prime},
$$

and $g(t)=\exp (t A)(t \in C, A \in \operatorname{Lie}(G))$ be a one-parameter subgroup of $G$. If $|t|$ is sufficiently small,

$$
g(t)^{*}\left(f^{\vee-\alpha} \omega^{\vee}\right)=\phi^{\alpha}(g(t))\left(f^{\vee-\alpha} \omega^{\vee}\right)
$$

by (3.13). Let $g(t)^{*} d z_{i}=\sum_{j=1}^{m} c_{i j} d z_{j}(1 \leq i \leq n)$, where $c_{i j}=c_{i j}(t ; z)$. Then (3.17.5) can be written by using the local coordinate as

$$
g(t)^{*} e\left(z^{\prime}\right) \cdot \operatorname{det}\left(c_{i j}\left(t ; z_{1}^{\prime}, \cdots, z_{m}^{\prime}, 0, \cdots, 0\right)\right)_{1 \leq i, j \leq m}=\phi^{\alpha}(g(t)) e\left(z^{\prime}\right) .
$$

Let $\dot{c}_{i j}=\dot{c}_{i j}(z)=\frac{\partial c_{i j}}{\partial t}(0 ; z)$ and $\left.\frac{d}{d t}\left(g(t)^{*} z_{i}\right)\right|_{t=0}=A z_{i}$. Then by differentiating (3.17.6), we get 


$$
\begin{aligned}
& \sum_{i=1}^{m}\left(A z_{i}\right) \frac{\partial e\left(z^{\prime}\right)}{\partial z_{i}^{\prime}}+\left(\sum_{i=1}^{m} \dot{c}_{i i}\right) e\left(z^{\prime}\right)=(\alpha \phi)(A) e\left(z^{\prime}\right), \\
& \left(f^{\vee-\infty} \omega^{\vee}\right)\left(-\sum_{i=1}^{m} \frac{\partial}{\partial z_{i}} \cdot\left(A z_{i}\right)+\sum_{i=1}^{m} \dot{c}_{i i}-(\alpha \phi)(A)\right)=0, \text { and }
\end{aligned}
$$

(3.17.8) $\quad\left(f^{\vee-\alpha} \omega^{\vee} \otimes 1_{O_{1}^{\vee} \rightarrow \Omega^{\vee}}\right)\left(-\sum_{i=1}^{m} \frac{\partial}{\partial z_{i}} \cdot\left(A z_{i}\right)+\sum_{i=1}^{m} \dot{c}_{i i}-(\alpha \phi)(A)\right)=0$

Let us show that (3.17.8) is nothing but (3.17.4). Let $g(t)^{*} z_{i}=\varphi_{i}\left(t ; z_{1}, \cdots, z_{n}\right)$. Then $c_{i j}=\frac{\partial \varphi_{i}}{\partial z_{j}}$ and $A z_{i}=\frac{\partial \varphi_{i}}{\partial t}(0 ; z)$. Hence

$$
\begin{aligned}
-\sum_{i=1}^{m} \frac{\partial}{\partial z_{i}} \cdot\left(A z_{i}\right)+\sum_{i=1}^{m} \dot{c}_{i i} \\
=-\sum_{i=1}^{m} \frac{\partial}{\partial z_{i}} \cdot \frac{\partial \varphi_{i}}{\partial t}(0 ; z)+\sum_{i=1}^{m} \frac{\partial^{2} \varphi_{i}}{\partial t \partial z_{i}}(0 ; z) \\
\quad=-\sum_{i=1}^{m} \frac{\partial \varphi_{i}}{\partial t}(0 ; z) \frac{\partial}{\partial z_{i}} .
\end{aligned}
$$

For $i>m, z_{i} \equiv 0$ on $O_{1}^{\vee}$. Since $O_{1}^{\vee}$ is $G$-stable, $g(t)^{*} z_{i} \equiv 0$ and $\frac{\partial \varphi_{i}}{\partial t}(0 ; z) \equiv 0$ on $O_{1}^{\vee}$. Since $1_{O_{1}^{\vee} \rightarrow \Omega^{\vee}}$ is annihilated by the defining ideal of $O_{1}^{\vee},(3.17 .8)$ can be written as

$$
\left(f^{\vee-\alpha} \omega^{\vee} \otimes 1_{O_{1}^{\vee} \rightarrow \Omega^{\vee}}\right)\left(-\sum_{i=1}^{n} \frac{\partial \varphi_{i}}{\partial t}(0 ; z) \frac{\partial}{\partial z_{i}}-(\alpha \phi)(A)\right)=0
$$

Let $y_{j}=\psi\left(z_{1}, \cdots, z_{n}\right)$. Then $g(t)^{*} y_{j}=\psi_{j}\left(g(t)^{*} z_{1}, \cdots, g(t)^{*} z_{n}\right)$, and

$$
-\sum_{i=1}^{n} a_{i j} y_{i}=\left.\frac{\partial}{\partial t}\left(g(t)^{*} y_{j}\right)\right|_{t=0}=\sum_{i=1}^{n} \frac{\partial \varphi_{i}}{\partial t}(0 ; z) \cdot \frac{\partial \psi_{j}}{\partial z_{i}}
$$

Hence

$$
\begin{aligned}
-\sum_{i=1}^{n} \frac{\partial \varphi}{\partial t}(0 ; z) \frac{\partial}{\partial z_{i}} & =-\sum_{i, j=1}^{n} \frac{\partial \varphi}{\partial t}(0 ; z) \cdot \frac{\partial y_{j}}{\partial z_{i}} \cdot \frac{\partial}{\partial y_{j}}=\sum_{i, j=1}^{n} a_{i j} y_{i} \frac{\partial}{\partial y_{j}} \\
& =\sum_{i, j=1}^{n} a_{j i} \frac{\partial}{\partial y_{i}} y_{j}-\sum_{i=1}^{n} a_{i i} .
\end{aligned}
$$

Hence (3.17.9) is nothing but (3.17.4).

Since $1_{O_{1}^{v} \rightarrow \Omega^{v}}$ is annihilated by $z_{m+1}, \cdots, z_{n}$, it would be more impressive to write 


$$
f^{\vee-\infty} h=\frac{f^{\vee-\alpha} \omega^{\vee} \wedge\left(\delta\left(z_{m+1}, \cdots, z_{n}\right) d z_{m+1} \wedge \cdots \wedge d z_{n}\right)}{d y_{1} \wedge \cdots \wedge d y_{n}} .
$$

3.18. Proof of (3.9.3). Since $1 \tilde{o}_{1 \rightarrow \tilde{\Omega}^{v}}$ is annihilated by the defining ideal $\mathcal{G}$ of $\tilde{O}_{1}^{\vee}, \mathcal{G}\left(\tilde{f}^{\vee-\alpha} \tilde{h}\right)=0$. Hence (3.17.3) induces a non-zero homomorphism

$$
\pi^{\vee-1} \mathscr{D}_{\Omega^{\vee}} u_{\alpha}^{\prime \prime} \rightarrow \int_{\tilde{i}^{\vee}} \mathscr{D}_{\tilde{o}_{1}^{\vee}} \tilde{f}^{\vee-\alpha}
$$

such that $u_{\alpha}^{\prime \prime} \rightarrow \tilde{f}^{\vee-\alpha} \tilde{h}$. Since $\mathscr{D} \tilde{o}_{1}^{\vee} \tilde{f}^{\vee-\alpha}$ is a simple $\mathscr{D}$-module, $\int \tilde{i}^{\vee} \mathscr{D}_{\tilde{o}_{1}^{\vee}} \tilde{f}^{\vee-\alpha}$ is also simple by [11; Chap.1, Theorem 5.1]. Hence (3.18.1) is a surjection. Since $\mathscr{D}^{a n} \otimes(3.18 .1)$ can be locally identified with a surjective, locally defined homomorphism

$$
\mathscr{D}_{\Omega^{\vee}}^{a n} u_{\alpha}^{\prime \prime} \rightarrow \int_{i} \mathcal{D}_{O_{1}^{\vee}}^{a n} f^{\vee-\alpha},
$$

we get the desired inclusion

$$
\operatorname{ch}\left(D\left(\Omega^{\vee}\right) u_{\alpha}^{\prime \prime}\right) \supset \operatorname{ch}\left(\int_{i} \mathscr{D}_{O_{1}^{\vee}}^{a n} f^{\vee-\infty}\right)=\left(T O_{1}^{\vee}\right)^{\perp}
$$

(Note that $\int_{i^{\vee}} \mathscr{D}_{O_{1}^{\vee}}^{a n} f^{\vee}-\alpha$ is locally isomorphic to $\mathscr{D}^{a n} \delta\left(z_{m+1}, \cdots, z_{n}\right)=\mathscr{D}^{a n} /\left(\sum_{k=1}^{m}\right.$ $\mathscr{D}^{a n} \frac{\partial}{\partial z_{k}}+\sum_{k=m+1}^{n} \mathscr{D}^{a n} z_{k}$ ) in terms of a local coordinate system $\left\{z_{1}, \cdots, z_{n}\right\}$ such that $\left\{z_{m+1}=\cdots=z_{n}=0\right\}=O_{1}^{\vee}$.)

\subsection{Regularity}

As is noted in (3.9), (3.9.3) implies the simplicity of $D\left(\Omega^{\vee}\right) u_{\alpha}^{\prime \prime}$. Hence (3.18.2) is a (locally defined) isomorphism. Hence (3.18.1) is an isomorphism. Since $\mathscr{D}_{\tilde{o}_{1}^{\vee}} \tilde{f}^{\vee-\alpha}$ is regular holonomic (cf. (2.8.6)), $\int_{\tilde{i}^{\vee}} \mathscr{D}_{\tilde{o}_{1}^{\vee}} \tilde{f}^{\vee-\infty}=\pi^{\vee-1} \mathscr{D}_{\Omega^{\vee}} u_{\alpha}^{\prime \prime}$ is also regular holonomic by [11; Chap. 4, Theorem 2.2.1]. Hence $D\left(\Omega^{\vee}\right) u_{a}^{\prime \prime}=$ $\left(D\left(V^{\vee}\right) u_{\alpha}^{\prime \prime}\right)\left[f^{\vee-1}\right]=D\left(\Omega^{\vee}\right) \mathscr{F}\left(f^{\alpha}\right)$ is a regular holonomic $D\left(\Omega^{\vee}\right)$-module. By (3.11, (3)), $\mathscr{F}\left(D f^{\alpha}\right)$ is a regular holonomic $D\left(V^{\vee}\right)$-module, if $\alpha \in A_{+}$. By (2.8.5), the composition factors of $\mathscr{F}\left(D f^{\alpha}\right)$ and $\mathscr{F}\left(D f^{\alpha+k}\right)$ are the same for any $k \in \mathbb{Z}$. Hence $\mathscr{F}\left(D f^{\alpha}\right)$ is a regular holonomic $D\left(V^{\vee}\right)$-module for any $\alpha$.

3.20. From the isomorphism (3.18.1), we get an injective homomorphism

$$
\mathscr{D}_{\Omega \vee} u_{\alpha}^{\prime \prime} \rightarrow \pi_{*}^{\vee} \pi^{\vee-1} \mathscr{H}(\alpha),
$$

where

$$
\mathscr{M}(\alpha)=\int_{i^{\vee}} \mathscr{D}_{O_{1}^{\vee}} f^{\vee-\alpha}
$$


Since $\Gamma=\operatorname{Gal}\left(\tilde{O}_{1}^{\vee} / O_{1}^{\vee}\right)$ is a cyclic group of order two, $\pi_{*}^{\vee} \pi^{\vee-1} \mathscr{H}(\alpha)$ decomposes into a direct sum of $\Gamma$-isotypic parts. Let $\mathscr{M}_{0}(\alpha)\left(\operatorname{resp} . \mathscr{M}_{1}(\alpha)\right)$ be the part corresponding to the trivial (resp. non-trivial) character of $\Gamma$. Note that $\mathscr{M}_{0}(\alpha)$ is the image of the canonical injection $\mathscr{M}(\alpha) \rightarrow \pi_{*}^{\vee} \pi^{\vee-1} \mathscr{M}(\alpha)$, and simple. Since $\mathscr{M}_{1}(\alpha)^{a n}$ is locally isomorphic to $\mathscr{H}(\alpha)^{a n}, \mathbb{C} \mathbb{h}\left(\mathscr{M}_{1}(\alpha)\right)=\left(T O_{1}^{\vee}\right)^{\perp}$ and $\mathscr{M}_{1}(\alpha)$ is simple. Since $\mathscr{D}_{\Omega^{v}} u_{\alpha}^{\prime \prime}$ is a simple submodule of $\pi_{*}^{v} \pi^{v-1} \mathscr{M}(\alpha)=\mathscr{M}_{0}(\alpha) \oplus \mathscr{M}_{1}(\alpha)$, and since $\mathscr{H}_{0}(\alpha)$ and $\mathscr{M}_{1}(\alpha)$ are simple,

$$
\mathscr{D}_{\Omega} \cdot u_{\alpha}^{\prime \prime} \simeq \mathscr{M}_{0}(\alpha) \text { or } \mathscr{M}_{1}(\alpha) \text {. }
$$

Lemma 3.21. $\mathscr{D}_{\Omega^{\vee}} \otimes_{D} \mathscr{F}\left(D f^{\alpha}\right) \simeq \mathscr{D}_{\Omega^{\vee}} u_{\alpha}^{\prime \prime} \simeq \mathscr{H}_{1}(\alpha) \simeq \pi_{*}^{\vee} \pi^{\vee-1} \mathscr{H}(\alpha) / \mathscr{H}(\alpha)$.

Proof. The first isomorphism follows from $(3.11,(5))$.

If $\omega^{\vee}$ is single-valued on the whole $O_{1}^{\vee}$, then $\tilde{O}_{1}^{\vee}$ is a disjonit union of two copies of $O_{1}^{\vee}$. Then the assertion is obvious.

Assume that $\mathscr{D}_{\Omega^{v}} u_{\alpha}^{\prime \prime} \simeq \mathscr{M}_{0}(\alpha)$. Since $\mathscr{M}_{0}(\alpha) \simeq \mathscr{M}(\alpha)$, we can find a global holomorphic $m$-form $\omega^{\prime}$ on $O_{1}^{\vee}$ such that $u_{\alpha}^{\prime \prime}$ corresponds to

$$
v_{\alpha}=v_{\alpha}(y)=\frac{f^{\vee-\alpha} \omega^{\prime} \otimes 1_{O_{1}^{\vee} \rightarrow \Omega^{\vee}}}{d y_{1} \wedge \cdots \wedge d y_{n}}
$$

by the above isomorphism. (Cf. (2.1.5) and (3.17.1).) Let $\left\{z_{1}, \cdots, z_{n}\right\}$ be a local coordinate system of $\Omega^{\vee}$ such that $O_{1}^{\vee}=\left\{z_{m+1}=\cdots=z_{n}=0\right\}, z_{i}^{\prime}=\left.z_{i}\right|_{o_{1}^{\vee}}(1 \leq i \leq$ $m$ ), and $f^{\vee-\alpha} \omega^{\prime}=e\left(z^{\prime}\right) d z_{1}^{\prime} \wedge \cdots \wedge d z_{m}^{\prime}$. Since $v_{\alpha}$ solves (3.5.1), $e\left(z^{\prime}\right)$ solves (3.17.7). ( $\operatorname{Read}$ (3.17) backward and apply (2.1.5).) Hence $f^{\vee-\infty} \omega^{\prime}$ is (locally defined) relatively invariant holomorphic $m$-form of character $\phi^{\alpha}$ with respect to $G$. Since $O_{1}^{\vee}$ is a single $G$-orbit, such an $m$-form is unique up to non-zero scalar multiple. Hence we may assume from the beginning that $\omega^{\mathrm{v} 2}=\omega^{\prime} \otimes \omega^{\prime}$. Hence $\omega^{\vee}$ is single-valued. Hence if $\omega^{\vee}$ is not single-valued, $\mathscr{D}_{\Omega^{\vee}} u_{a}^{\prime \prime}$ is not isomorphic to $\mathscr{M}_{0}(\alpha)$. Thus the assertion follows from (3.20.3).

3.22. Let $L(\alpha)=C f^{\alpha}$ and $L^{\vee}(\alpha)=\mathbb{C}\left(f^{\vee}\right)^{\alpha}$ be the locally constant sheaves of rank 1 on $\Omega$ and $\Omega^{\vee}$ generated by (local) single-valued branches of $f^{\alpha}$ and $\left(f^{\vee}\right)^{\alpha}$. Decompose the locally constant sheaf $\pi_{*}^{\vee} C_{\widetilde{o}_{1}^{\vee}}$ into $\Gamma$-isotypic parts $\mathbb{C}_{O_{1}^{\vee}}$ and $H^{\vee}$, where $\boldsymbol{C}_{O_{1}^{\vee}}$ (resp. $H^{\vee}$ ) corresponds to the trivial (resp. non-trivial) character of $\Gamma$. (Recall that $\Gamma=\operatorname{Gal}\left(\tilde{O}_{1}^{\vee} / O_{1}^{\vee}\right)$.) Then $\underline{\operatorname{Hom}}_{C}\left(H^{\vee}, \mathbb{C}\right)=H^{\vee}$. By (3.20.2),

$$
\begin{aligned}
& \operatorname{Sol}\left(\pi_{*}^{\vee} \pi^{\vee-1} \mathcal{H}(\alpha)\right)=\pi_{*}^{\vee} \pi^{\vee}-1 i_{*}^{\vee} L^{\vee}(-\alpha)[m-n]=i_{*}^{\vee} \pi_{*}^{\vee} \pi^{\vee-1} L^{\vee}(-\alpha)[m-n] \\
& =i_{*}\left(L^{\vee}(-\alpha) \otimes_{C^{*}} \pi_{*}^{\vee} \pi^{\vee-1} \mathbb{C}_{O_{1}^{\vee}}\right)[m-n]
\end{aligned}
$$


Here we have written $L^{\vee}(-\alpha)$ for $\left.L^{\vee}(-\alpha)\right|_{o_{1}^{\vee}}$. Hence

$$
\begin{aligned}
& \operatorname{Sol}\left(\mathscr{M}_{1}(\alpha)\right)=i_{*}^{\vee}\left(L^{\vee}(-\alpha) \otimes H^{\vee}\right)[m-n], \quad \text { and } \\
& \operatorname{DR}\left(\mathscr{M}_{1}(\alpha)\right)=i_{*}^{\vee}\left(L^{\vee}(\alpha) \otimes H^{\vee}\right)[m-n] .
\end{aligned}
$$

Theorem 3.23. Let $j: \Omega \rightarrow V, j^{\vee}: \Omega^{\vee} \rightarrow V^{\vee}, i: O_{1} \rightarrow \Omega$ and $i^{\vee}: O_{1}^{\vee} \rightarrow \Omega^{\vee}$ be the inclusion mappings, $n=\operatorname{dim} V=\operatorname{dim} V^{\vee}$ and $m=\operatorname{dim} O_{1}=\operatorname{dim} O_{1}^{\vee}$.

(1) $\operatorname{Sol}\left(D f^{\alpha}\right)=R j_{*} L(\alpha)$, if $\alpha \in A_{+}$.

(2) $\operatorname{Sol}\left(D f^{\alpha}\right)=j_{!} L(\alpha)$, if $\alpha \in A_{\text {. }}$.

(3) $\operatorname{Sol}\left(\mathscr{F}\left(D f^{\alpha}\right)\right)=j_{!}^{\vee} i_{*}^{\vee}\left(L^{\vee}(-\alpha) \otimes H^{\vee}\right)[m-n]$, if $\alpha \in A_{+}$.

(4) $\operatorname{Sol}\left(\mathscr{F}\left(D f^{\alpha}\right)\right)=R j_{*}^{\vee} i_{*}^{\vee}\left(L^{\vee}(-\alpha) \otimes H^{\vee}\right)[m-n]$, if $\alpha \in A_{-}$.

(5) $\operatorname{DR}\left(D f^{\alpha}\right)=j_{1} L(-\alpha)$, if $\alpha \in A_{+}$.

(6) $\operatorname{DR}\left(D f^{\alpha}\right)=R j_{*} L(-\alpha)$, if $\alpha \in A_{-}$.

(7) $\mathrm{DR}\left(\mathscr{I}\left(D f^{\alpha}\right)\right)=R j_{*}^{\vee} i_{*}^{\vee}\left(L^{\vee}(\alpha) \otimes H^{\vee}\right)[m-n]$, if $\alpha \in A_{+}$.

(8) $\mathrm{DR}\left(\mathscr{F}\left(D f^{\alpha}\right)\right)=j_{1}^{\vee} i_{*}^{\vee}\left(L^{\vee}(\alpha) \otimes H^{\vee}\right)[m-n]$, if $\alpha \in A_{-}$.

Proof. By (2.8.6) and (3.19), $D f^{\alpha}$ and $\mathscr{F}\left(D f^{\alpha}\right)$ are regular holonomic. Hence we can use the commutativity of DR with other functors (cf. [11; Chap. 5]). If $\alpha \in A_{-}$,

$$
\begin{aligned}
\operatorname{DR}\left(D f^{\alpha}\right) & =\operatorname{DR}\left(D f^{\alpha}\left[f^{-1}\right]\right), \quad \text { by }(3.11,(1)) \\
& =R j_{*} L(-\alpha) .
\end{aligned}
$$

If $\alpha \in A_{+}$.

$$
\begin{aligned}
\operatorname{DR}\left(D f^{\infty}\right) & =\operatorname{DR}\left(\left(D f^{-\infty}\right)\left[f^{-1}\right]^{*}\right), \quad \text { by }(3.11,(2)) \\
& =j_{!} L(-\alpha) .
\end{aligned}
$$

If $\alpha \in A_{+}$,

$$
\begin{array}{rlrl}
\operatorname{DR}\left(\mathscr{F}\left(D f^{\alpha}\right)\right) & =\operatorname{DR}\left(\mathscr{F}\left(D f^{\alpha}\right)\left[f^{\vee-1}\right]\right), & & \text { by }(3.11,(3)) \\
& =\operatorname{DR}\left(\left(D u_{\alpha}^{\prime \prime}\right)\left[f^{\vee-1}\right]\right), & & \text { by }(3.11,(5)) \\
& =R j_{*}^{\vee} \operatorname{DR}\left(\left.D u_{\alpha}^{\prime \prime}\right|_{\Omega^{\vee}}\right) & \\
& =R j_{*}^{\vee} \operatorname{DR}\left(\mathscr{M}_{1}(\alpha)\right), & & \text { by }(3.21) \\
& =R j_{*}^{\vee} i_{*}^{\vee}\left(L^{\vee}(\alpha) \otimes H^{\vee}\right)[m-n], & & \text { by }(3.22) .
\end{array}
$$

If $\alpha \in A_{-}$,

$$
\begin{aligned}
\operatorname{DR}\left(\mathscr{F}\left(D f^{\alpha}\right)\right) & =\operatorname{DR}\left(\mathscr{F}\left(D f^{-\alpha}\right)\left[f^{\vee-1}\right]^{*}\right), & & \text { by }(3.11,(4)) \\
& =\operatorname{DR}\left(\left(D u_{-\alpha}^{\prime \prime}\right)\left[f^{\vee-1}\right]^{*}\right), & & \text { by }(3.11,(5)) \\
& =j_{!}^{\vee} \operatorname{DR}\left(\left.\left(D u_{-\alpha}^{\prime \prime}\right)^{*}\right|_{\Omega^{\vee}}\right) & & \\
& =j_{!}^{\vee} i_{*}^{\vee}\left(L^{\vee}(\alpha) \otimes H^{\vee}\right)[m-n], & & \text { by (3.21) and (3.22). }
\end{aligned}
$$


Thus we get (5)-(8). The assertions (1)-(4) can be obtained from (5)-(8) by the duality of DR and Sol. Cf. [11; Chap.5, 2.1. (ii) and 3.3.(i)].

Example 3.24. Let $(G, \rho, V)$ be the prehomogeneous vector space considered in (1.20). Then $V$ has ten $G$-orbits:
(I) (II)
(III) (IV)
(V)
(0) $E_{1,1}+E_{2,2}+E_{m+1,3}$
$3 \quad\left(C_{m-2} T_{1}\right) U_{2 m-3}$
$6 m$
(1) $E_{1,1}+E_{m+1,3}$
$2 \quad\left(C_{m-1} T_{1}\right)$
$4 m+2$
(2) $E_{1,1}+i E_{1,3}+E_{m+1,2}+E_{m+2,3}$
$3 \quad\left(C_{m-2} T_{1}\right) U_{2 m-2}$
$6 m-1$
(3) $E_{1,1}+i E_{1,3}+E_{m+1,2}$
$2 \quad\left(C_{m-1} T_{1}\right) U_{1}$
$4 m+1$
(4) $E_{1,1}+E_{2,2}+E_{3,3}$
$3 \quad\left(C_{m-3} A_{1} T_{1}\right) U_{6 m-12}$
$6 m-3$
(5) $E_{1,1}+E_{2,2}$
$2 \quad\left(C_{m-2} T_{2}\right) U_{4 m-5}$
$4 m+1$
(6) $E_{1,1}+i E_{1,2}$
$1 \quad\left(C_{m-1} T_{2}\right) U_{2 m}$
$2 m+1$
(7) $E_{1,1}+i E_{1,2}+E_{2,3}$
$2 \quad\left(C_{m-2} T_{2}\right) U_{4 m-4}$
$4 m$
(8) $E_{1,1}$
$1 \quad\left(C_{m-1} T_{2}\right) U_{2 m-1}$
$2 m+2$
(9) 0
$0 \quad\left(C_{m} A_{1} T_{1}\right)$
0

Here the second column contains a representative element $X_{j}$ of each orbit $O_{j}(0 \leq j \leq 9)$, where $E_{j k}$ denotes the matrix unit and $i=\sqrt{-1}$. The third column contains the rank of $X_{\boldsymbol{j}}$. The fourth column contains the local structure of the isotropy subgroup at $X_{i}$, where $A_{k}$ (resp. $C_{k}$ ) denotes the simple Lie group of type $A_{k}$ (resp. $C_{k}$ ), $T_{k}$ denotes the torus of $k$ dimension, and $U_{k}$ denotes a $k$-dimensional unipotent group. The last column contains the dimension of the orbit.

Let $\Lambda_{j}$ be the Zariski closure in $V \times V^{\vee}$ of the conormal bundle of $O_{j}$. To each $\Lambda_{j}$ associate a vertex $j$. Connect two vertexes $j$ and $k$ by a solid line if

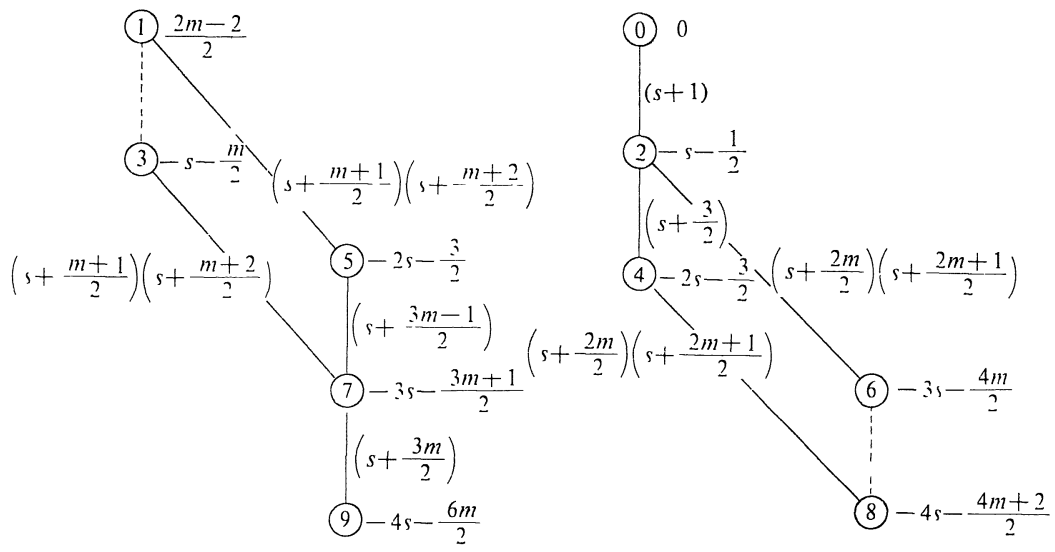


$\Lambda_{j} \cap \Lambda_{k}$ contains a $G$-orbit whose dimension is $\operatorname{dim} V-1$. Connect them by a broken line if $\operatorname{dim} \Lambda_{j} \cap \Lambda_{k}=\operatorname{dim} V-1$ and $\Lambda_{j} \cap \Lambda_{k}$ does not contain a $G$-orbit whose dimension is $\operatorname{dim} V-1$. Thus we obtain the following diagram, which is called the holonomy diagram of $(G, \rho, V)$. (See [25] for holonomy diagrams, etc.)

Let $(G, \rho, V)$ be a general prehomogeneous vector space, $n=\operatorname{dim} V$, $\left\{v_{1}, \cdots, v_{n}\right\}$ a basis of $V$ and $\left\{v_{1}^{\vee}, \cdots, v_{n}^{\vee}\right\}$ its dual basis. For $A \in \operatorname{Lie}(G)$, let $\rho(A) v_{j}=\sum_{i=1}^{n} v_{i} a_{i j}$ and $N^{\prime}(\alpha)=D u_{\alpha}^{\prime}$ the $D$-module defined by

$$
\left(\sum_{i, j=1}^{n} a_{i j} x_{j} \frac{\partial}{\partial x_{\dot{i}}}-\alpha \phi(A)\right) v_{\alpha}^{\prime}=0
$$

Now let us return to the above example. Then $\operatorname{ch}\left(N^{\prime}(\alpha)\right) \subset \cup_{i=0}^{9} \Lambda_{i}$ (cf. [15; 5.1.12]). Since $O_{0}$ is the open $G$-orbit in $V, \Lambda_{0} \subset \operatorname{ch}\left(D f^{\alpha}\right)$. (See (2.3.1) for $D f^{\alpha}$.) By $[25 ; 6.6]$ and by the above holonomy diagram, we can show that $\Lambda_{i} \subset \operatorname{ch}(N)$ for even $i$. (See (2.3.1) for $N=D[s] f^{s}$.) Since scalar multiplications are contained in $\rho(G)$, we can find an element $A_{1} \in \operatorname{Lie}(G)$ such that $\rho\left(A_{1}\right)$ is the identity. Then by $(1.9,(1))$,

$$
s d=\langle x \mid s \cdot \operatorname{grad} \log f(x)\rangle=\left\langle\rho\left(A_{1}\right) x \mid s \cdot \operatorname{grad} \log f(x)\right\rangle .
$$

Here $d=\operatorname{deg} f=4$. In the notation of $[25 ; 4.2],(S)$ equals $\sigma(x, s \cdot \operatorname{grad} \log f(x))$. Hence the pull-back of $\sigma$ to $\tilde{W}$ is equal to $s \cdot d$. Hence

$$
\begin{aligned}
\operatorname{ch}\left(D f^{\alpha}\right) & =p\left(\tilde{W}_{0}\right), \quad \text { by }(2.4 .5) \text { and }(2.4 .6) \\
& =p(\{(s, x, y) \in \tilde{W} \mid \sigma(x, y)=0\}) \\
& =\{(x, y) \in W \mid \sigma(x, y)=0\}, \quad \text { by (2.4.4) }
\end{aligned}
$$

Since $\sigma \equiv 0$ on any $\Lambda_{i}$ (cf. $\left.[25 ; 4.4]\right), \Lambda_{i} \subset \operatorname{ch}(N)(=W) \Leftrightarrow \Lambda_{i} \subset \operatorname{ch}\left(D f^{\alpha}\right)$. Hence $\Lambda_{i} \subset \operatorname{ch}\left(D f^{\alpha}\right)$ for even $i$. The Fourier transform of $N^{\prime}(\alpha)$ is the $D$-module defined by

$$
\left(\sum_{i, j=1}^{n}\left(-a_{j \dot{z}}\right) y_{j} \frac{\partial}{\partial y_{i}}-\left(\alpha \phi+\phi_{0}\right)(A)\right) u_{\alpha}^{\prime}=0
$$

If $A=\left(t, A^{\prime}, A^{\prime \prime}\right) \in \operatorname{Lie}\left(C^{\times} \times S p_{2 m} \times S O_{3}\right)$, then $\phi(A)=4 t$ and $\phi_{0}(A)=6 m t=$ $(6 m / 4) \phi(A)$. Hence $D f^{\vee-\alpha-6 m / 4}$ is a quotient of $\mathscr{F}\left(N^{\prime}(\alpha)\right)$ and $\mathscr{H}\left(D f^{\vee-\alpha-6 m / 4}\right)$ is a quotient of $N^{\prime}(\alpha)$. Since $\Lambda_{9}$ is the conormal bundle of the open orbit $O_{0}^{\vee}$ of $V^{\vee}$, we can show that $\Lambda_{i} \subset \operatorname{ch}\left(D f^{\vee-\alpha-6 m / 4}\right)$ for odd $i$ by the same argument as above. By (2.7.2), $\Lambda_{i} \subset \operatorname{ch}\left(\mathscr{F}\left(D f^{\vee-\alpha-6 m / 4}\right)\right)$ for odd $i$. Since $D f^{\alpha}$ and $\mathscr{F}\left(D f^{\vee-\alpha-6 m / 4}\right)$ are both quotients of $N^{\prime}(\alpha)$, we have the natural morphism 


$$
N^{\prime}(\alpha) \rightarrow D f^{\alpha} \oplus \mathscr{F}\left(D f^{\vee-\alpha-6 m / 4}\right) .
$$

By a direct calculation (cf. $[25 ; 4.4]$ ), we can show that every $\Lambda_{i}$ is $G$-prehomogeneous. Hence, as in $[25 ; 4.8]$, we can show that

$$
\begin{aligned}
& m\left(\Lambda_{i}, N^{\prime}(\alpha)\right)=m\left(\Lambda_{i}, D f^{\alpha}\right)=1 \text { for even } i, \text { and } \\
& m\left(\Lambda_{i}, N^{\prime}(\alpha)\right)=m\left(\Lambda_{i}, D f^{\vee-\alpha-6 m / 4}\right)=1 \text { for odd } i .
\end{aligned}
$$

By considering the support, we can show that $\operatorname{ch} D f^{\alpha}=\operatorname{ch} \mathscr{F}\left(D f^{\alpha}\right) \perp \Lambda_{9}$ (resp. $\left.\operatorname{ch} \mathscr{I}\left(D f^{\vee-\alpha-6 m / 4}\right) \perp \Lambda_{0}\right)$, which implies that $\Lambda_{i}$ for odd $i$ (resp. even $i$ ) is not contained in this characteristic variety. Hence

$$
N^{\prime}(\alpha)=D f^{\alpha} \oplus \mathscr{F}\left(D f^{\vee-\alpha-6 m / 4}\right) .
$$

By (1.6) and (3.1),

(3.24.3) $f^{\vee}(\partial) f \cdot f^{\alpha}=b(\alpha) f^{\alpha}$, and

(3.24.4) $f^{\vee}(\partial) f \circ \mathscr{I}\left(f^{\vee-\alpha-6 m / 4}\right)=(-1)^{4} b(-\alpha-6 m / 4-1) \mathscr{F}\left(f^{\vee-\alpha-6 m / 4}\right)$.

The $b$-function appeared in (3.24.3) can be calculated using the right half of the holonomy diagram (cf. [25; §7]):

$$
b(s)=2^{4}(s+1)\left(s+\frac{3}{2}\right)\left(s+\frac{2 m}{2}\right)\left(s+\frac{2 m+1}{2}\right) .
$$

(See [30; Prop.2.7] for the way of calculation of the leading coefficient of $b(s)$.) The other ' $b$-function' appeared in (3.24.4) can be calculated using the left half of the holonomy diagram:

$$
(-1)^{4} b\left(-s-\frac{6 m}{4}-1\right)=2^{4}\left(s+\frac{3 m}{2}\right)\left(s+\frac{3 m-1}{2}\right)\left(s+\frac{m+2}{2}\right)\left(s+\frac{m+1}{2}\right) .
$$

The inclusion relations between closures of orbits $O_{j}(0 \leq j \leq 0)$ are given by the following diagram:

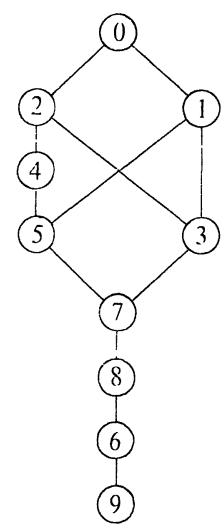


Here the vertex $j$ lies under $k$ if and only if $O_{j}$ is contained in the closure of $O_{k}$. For example

$$
\bar{O}_{1}=\bigcup_{j \neq 0,2,4} O_{j}=\left\{X \in V=M_{2 m, 3}(C) \mid \operatorname{rank}(X) \leq 2\right\}
$$

\section{§4. Relatively Invariant Hyperfunctions}

4.0. The purpose of this section is to prove (4.19), which describes the hyperfunction solutions of $D f^{a}$ and their Fourier transforms. (See (2.1.6) for 'solution'.)

\subsection{Hyperfunctions}

Let $X$ be an $n$-dimensional smooth algebraic variety defined over $\boldsymbol{R}$. By the implicit function theorem, $X(\mathbb{R})$ is an $n$-dimensional real analytic submanifold of the complex manifold $X(\boldsymbol{C})$. (Here and below, $X(K)$ denotes the set of $K$-rational points.) Let $\mathcal{O}^{a n}=\mathcal{O}_{X}^{a n}$ be the sheaf of holomorphic functions on $X(\boldsymbol{C}), \boldsymbol{C}=\boldsymbol{C}_{X}$ the constant sheaf, $\mathcal{A}=\mathcal{A}_{X}=\left.\mathcal{O}_{X}^{a n}\right|_{X(\boldsymbol{R})}$, and

$$
\mathscr{B}=\mathscr{B}_{X}=\left.R \underline{\operatorname{Hom}}_{C_{X}}\left(R \Gamma_{X(R)}\left(C_{X}\right), R \Gamma_{X(R)}\left(\mathcal{O}_{X}^{a n}\right)\right)\right|_{X(R)} .
$$

Then $\mathscr{B}$ is locally isomorphic to $\left.R \Gamma_{X(\boldsymbol{R})}\left(\mathcal{O}_{X}^{a n}\right)[n]\right|_{X(\boldsymbol{R})}$. It is known (cf. [17; Chap.2, §2]) that $H^{i}(\mathscr{B})=0(i \neq 0)$. Hence we can identify $\mathscr{B}$ with the sheaf $H^{0}(\mathscr{B})$. A local section of $\mathcal{B}$ is called a hyperfunction. Since

$$
\mathscr{A}=\left.\underline{\operatorname{Hom}}_{C_{X}}\left(C_{X}, \mathcal{O}_{X}^{a n}\right)\right|_{X(R)},
$$

we have a natural homomorphism $\mathscr{A} \rightarrow \mathscr{B}$, which is known to be injective (cf. [17; Chap.2, §3]). By this injection, we regard a real analytic function as a hyperfunction. It is also known that any distribution can be regarded as a hyperfunction.

\subsection{Real form}

Let $K$ be a real number field $\boldsymbol{R}$ or the complex number field $\boldsymbol{C}, G_{K}$ a reductive group scheme over $\operatorname{spec}(K), V_{K}=\operatorname{spec} K\left[x_{1}, \cdots, x_{n}\right]$, and $\rho_{K}: G_{K} \rightarrow G L_{n, K}$ $=\operatorname{spec}\left(K\left[x_{i j}(1 \leq i, j, \leq n), \operatorname{det}\left(x_{i j}\right)^{-1}\right]\right)$ a homomorphism of group schemes. We denote the set of rational points of $V_{K}$ etc. by $V_{K}(K)$ or $V(K)$ etc. If $K$ is an algebraically closed field, we sometimes identify $V_{K}$ etc. with $V(K)$ etc., but otherwise, we do not do such an identification. If $\left(G_{R}, \rho_{R}, V_{R}\right) \otimes C$ is isomorphic to $\left(G_{\boldsymbol{C}}, \rho_{\boldsymbol{C}}, V_{\boldsymbol{C}}\right)$, we call $\left(G_{\boldsymbol{R}}, \rho_{\boldsymbol{R}}, V_{\boldsymbol{R}}\right)$ a real form of $\left(G_{\boldsymbol{C}}, \rho_{\boldsymbol{C}}, V_{\boldsymbol{C}}\right)$. (Here $G_{\boldsymbol{R}} \otimes \boldsymbol{C}$ $=G_{\boldsymbol{R}} \times_{\mathrm{spec}(\boldsymbol{R})} \operatorname{spec}(\boldsymbol{C})$ etc. $)$ 
Let $\phi_{C}$ be a character of $G_{C}$. Then there exists a real form $\left(G_{R}, \rho_{\mathbb{R}}, V_{\mathbb{R}}\right)$ and a character $\phi_{\mathbb{R}}: G_{\mathbb{R}} \rightarrow G L_{1, \mathbb{R}}=\operatorname{spec}\left(\mathbb{R}\left[t, t^{-1}\right]\right)$ such that $\phi_{\mathbb{R}} \otimes C=\phi_{C}$. For example take any split $\mathbb{R}$-form of $\left(G_{C}, \rho_{C}, V_{C}\right)$ [5].

\subsection{Prehomogeneous vector spaces defimed over $\mathbb{R}$}

Let $\left(G_{C}, \rho_{C}, V_{C}\right)$ be a prehomogeneous vector space, $f$ a relative invariant of character $\phi_{C}$, and take a real form $\left(G_{\mathbb{R}}, \rho_{R}, V_{R}\right)$ such that $G_{\mathbb{R}}$ has a character $\phi_{\mathbb{R}}$ such that $\phi_{\mathbb{R}} \otimes \mathbb{C}=\phi_{C}$. Henceforth, we omit the suffixes $\mathbb{R}$ and $\mathbb{C}$ if there is no fear of confusion. Take vector basis $\left\{v_{1}^{\vee}, \cdots, v_{n}^{\vee}\right\}$ of $V^{\vee}(\mathbb{R})$. Let $x_{\dot{8}}=v_{i}^{\vee}$, and

$$
f(x)=\sum a_{\dot{s}_{1}, \cdots, i_{n}} x_{1^{1000}}^{i_{n}} x_{n}^{i_{n}} \quad\left(a_{i_{1}, \cdots, i_{n}} \in \mathbb{C}\right) .
$$

Define its complex conjugate by

$$
f^{c}(x)=\sum a_{i_{1}, \cdots, i_{n}}^{c} x_{1}^{i_{1} \cdots x_{n}} x_{n}^{i_{n}},
$$

where ${ }^{c}$ denotes the complex conjugation. Then $f^{c}$ is also a relative invariant of character $\phi$. Hence if one of its coefficients is real, then $f(x)=f^{c}(x) \in$ $\mathbb{R}\left[x_{1} \cdots, x_{n}\right]$. Hence by multiplying a suitable scalar, we may assume from the beginning that $f(x) \in \mathbb{R}\left[x_{1}, \cdots, x_{n}\right]$. Let $\left\{y_{1}, \cdots, y_{n}\right\}$ be the dual coordinate system of $\left\{x_{1}, \cdots, x_{n}\right\}$ and $f^{\vee}$ a relative invariant on the dual prehomogeneous vector space $\left(G, \rho^{\vee}, V^{\vee}\right)$ of character $\phi^{-1}$. By the same reason as above, we assume that $f^{\vee}(y) \in \mathbb{R}\left[y_{1}, \cdots, y_{n}\right]$ without loss of generality.

4.4. Let us consider $\Omega(\mathbb{R}), \Omega^{\vee}(\mathbb{R}), O_{1}(\mathbb{R})$ and $O_{1}^{\vee}(\mathbb{R})$. (Recall that $\Omega=$ $f^{-1}\left(\mathbb{C}^{\times}\right)$and $\Omega^{\vee}=f^{\vee-1}\left(\mathbb{C}^{\times}\right)$, and see (1.4) for $O_{1}$ and $O_{1}^{\vee}$ ) Let $F=\operatorname{grad} \log f$ and $F^{\vee}=\operatorname{grad} \log f^{\vee}$ as before. By (1.8),

$$
O_{1}^{\vee}(\mathbb{R})=F F^{\vee}\left(O_{1}^{\vee}(\mathbb{R})\right) \subset F\left(O_{1}(\mathbb{R})\right) \subset F(\Omega(\mathbb{R})) \subset O_{1}^{\vee}(\mathbb{R})
$$

Hence

$$
O_{1}^{\vee}(\mathbb{R})=F\left(O_{1}(\mathbb{R})\right)=F(\Omega(\mathbb{R}))
$$

Since $\Omega(\mathbb{R}) \neq \phi, O_{1}^{\vee}(\mathbb{R}) \neq \phi$. Hence $O_{1}^{\vee}(\mathbb{R})$ is a real analytic manifold of dimension $m\left(=\operatorname{dim} O_{1}^{v}\right)$. Similarly, $O_{1}(\mathbb{R})$ is also a real analytic manifold of dimension $m$. Let

$$
\Omega(\mathbb{R})=\bigcup_{j=1}^{l^{\prime}} \Omega_{j} \quad \text { and } \quad \Omega^{\vee}(\mathbb{R})=\bigcup_{j=1}^{l^{\prime \prime}} \Omega_{j}^{\vee}
$$

be the decomposition into connected components.

Lemma $4.5 \% \Omega_{j} \cap O_{1}=F^{\vee} F\left(\Omega_{j}\right)$. 
Proof. Let $v \in \Omega_{j}$. By (1.18), $F^{-1}(F(v))$ is an affine subspace of $V$ containing v. Hence $F^{-1}(F(v)) \cap V(\boldsymbol{R}) \subset \Omega_{j}$. By $(1.18), \quad F F^{\vee} F(v)=F(v)$, i.e., $F^{\vee} F(v) \in F^{-1}(F(v))$. Since $F^{\vee} F(v) \in O_{1}(\boldsymbol{R})$ by $(1.18), F^{\vee} F(v) \in F^{-1}(F(v)) \cap$ $O_{1}(\boldsymbol{R}) \subset \Omega_{j} \cap O_{1}$. On the other hand, since $F^{\vee} F$ is the identity on $O_{1}$ by (1.18), $\Omega_{j} \cap O_{1}=F^{\vee} F\left(\Omega_{j} \cap O_{1}\right) \subset F^{\vee} F\left(\Omega_{j}\right)$.

Lemma 4.6. The decomposition of $O_{1}(\boldsymbol{R})$ and $O_{1}^{\vee}(\boldsymbol{R})$ into connected components are given by $O_{1}(\boldsymbol{R})=\bigcup_{j=1}^{l^{\prime}}\left(\Omega_{j} \cap O_{1}\right)$, and $O_{1}^{\vee}(\boldsymbol{R})=\bigcup_{j=1}^{l^{\prime \prime}}\left(\Omega_{j}^{\vee} \cap O_{1}^{\vee}\right)$.

Proof. By (4.5), $\Omega_{j} \cap O_{1}\left(1 \leq j \leq l^{\prime}\right)$ are connected and non-empty. Since $\Omega_{j}$ are open subsets of $\Omega(\mathbb{R}), \Omega_{j} \cap O_{1}$ are open subsets of $\Omega(\boldsymbol{R}) \cap O_{1}=O_{1}(\boldsymbol{R})$. Since the above union is disjoint, we get the assertion.

4.7. Since $O_{1}(\mathbb{R})$ is a homeomorphic to $O_{1}^{\vee}(\mathbb{R})$ by $F$, their connected components are in natural one-to-one correspondence, i.e., $l^{\prime}=l^{\prime \prime}$. Let $l=l^{\prime}=$ $l^{\prime \prime}$ and assume that

$$
F\left(\Omega_{j} \cap O_{1}\right)=\Omega_{j}^{\vee} \cap O_{1}^{\vee}
$$

Since $F\left(\Omega_{j}\right)$ is connected subset of $O_{1}^{\vee}(\boldsymbol{R})$ and contains $\Omega_{j}^{\vee} \cap O_{1}^{\vee}$ by (4.7.1), we have

$$
F\left(\Omega_{j}\right)=\Omega_{j}^{\vee} \cap O_{1}^{\vee}
$$

Lemma 4.8. Let $G(\boldsymbol{R})^{+}$be the identity component of $G(\boldsymbol{R})$. Then $\Omega_{j} \cap O_{1}$ and $\Omega_{j}^{\vee} \cap O_{1}^{\vee}$ are $G(\mathbb{R})^{+}$-orbits.

Proof. For any $v \in O_{1}(\boldsymbol{R}), g \rightarrow g v$ defines a submersion $G(\boldsymbol{R}) \rightarrow O_{1}(\boldsymbol{R})$. Hence $G(\boldsymbol{R})^{+} v$ are connected open subsets of $O_{1}(\boldsymbol{R})$, and $O_{1}(\boldsymbol{R})$ is their disjoint union. Hence each connected component $\Omega_{j} \cap O_{1}$ of $O_{1}(\boldsymbol{R})$ is a $G(\boldsymbol{R})^{+}$-orbit.

Lemma 4.9. (1) $\left|f^{\vee}(y)\right|^{\alpha}$ is a real analytic function on each $\Omega_{j}^{\vee}$. (2) For $g \in G(\boldsymbol{R})^{+}$and $v^{\vee} \in \Omega_{j},\left|f^{\vee}\left(g v^{\vee}\right)\right|^{\alpha}=\phi(g)^{-\alpha}\left|f^{\vee}\left(v^{\vee}\right)\right|^{\alpha}$.

Proof. By (1.8), $b_{0} f^{\vee}(y)^{-1}=f\left(F^{\vee}(y)\right)$ on $\Omega_{1}^{\vee}$. Since the signature of $f$ is constant on $F^{\vee}\left(\Omega_{j}^{\vee}\right)=\Omega_{j} \cap O_{1}$ (cf. (4.7.2)), the signature of $f^{\vee}(y)$ on $\Omega_{j}$ is also constant. (Note that $b_{0} \neq 0$ by (1.7).) Hence we get (1). The second assertion is obvious.

4.10. Let $\omega$ be the holomorphic $m$-form defined locally on $O_{1}$ given in (3.14). Since $\omega \otimes \omega$ is globally single-valued and does not vanish at any point of $O_{1},|\omega|$ gives a real analytic $m$-form on $O_{1}(\boldsymbol{R})$. Hence $\left|F^{\vee} * \omega\right|$ gives a real analytic $m$-form on $\Omega^{\vee}(\boldsymbol{R})$. (Here our use of the term ' $m$-form' is slightly abu- 
sive. The author hopes that it does not cause any confusion.)

Let $\left\{z_{1}, \cdots, z_{n}\right\}$ be a local coordinate system of $V^{\vee}(\boldsymbol{R})$ such that $\left\{z_{m+1}=\right.$ $\left.\cdots=z_{n}=0\right\}=O_{1}^{\vee}(\mathbb{R})$. Then the $(n-m)$-form

$$
\left|\delta\left(z_{m+1}, \cdots, z_{n}\right) d z_{m+1} \wedge \cdots \wedge d z_{n}\right|:=\delta\left(z_{m+1}, \cdots, z_{n}\right)\left|d z_{m+1} \wedge \cdots \wedge d z_{n}\right|
$$

determined by the delta function of Dirac does not depend on the choice of a local coordinate system. See [17; Prop. 2.4.1] and the remark following it.

Define a hyperfunction $h^{\vee}$ on $\Omega^{\vee}(\mathbb{R})$ by

$$
h^{\vee}=\left|\frac{F^{\vee} \omega \Lambda \wedge \delta\left(z_{m+1}, \cdots, z_{n}\right)\left(d z_{m+1} \wedge \cdots \wedge d z_{n}\right)}{d y_{1} \wedge \cdots \wedge d y_{n}}\right| .
$$

Define a hyperfunction $\left|f^{\vee}\right| \bar{j}^{-\alpha} \cdot h^{\vee}$ on $\Omega^{\vee}(\mathbb{R})$ by

$$
\left|f^{\vee}\right|_{j}^{-\alpha} \cdot h^{\vee}= \begin{cases}\left|f^{\vee}\right|^{-\alpha} \cdot h^{\vee}, & \text { on } \Omega_{j}^{\vee} \\ 0, & \text { on } \Omega^{\vee}(\mathbb{R})-\Omega_{1}^{\vee} .\end{cases}
$$

By $(4.9,(1))$, the product $\left|f^{\vee}\right|^{-\alpha} h^{\vee}$ has a meaning on $\Omega_{j}^{\vee}$.

Lemma 4.11. The hyperfunctions $\left|f^{\vee}\right|_{j}^{-\alpha} \cdot h^{\vee}(1 \leq j \leq l)$ on $\Omega^{\vee}(\mathbb{R})$ are solutions of (3.5.1) and (3.5.2).

Proof. The numerator of $h^{\vee}$ is absolutely invariant with respect to $G(\mathbb{R})^{+}$. The denominator $\left|d y_{1} \wedge \cdots \wedge d y_{n}\right|$ is relatively invariant, and the corresponding character is $\operatorname{det} \rho^{\vee}(g)=\phi_{0}(g)^{-1}$. Since $\left|f^{\vee}\right|^{-\infty}$ corresponds to the character $\alpha \phi,\left|f^{\vee}\right|_{j}^{-\alpha} \circ h^{\vee}$ corresponds to $\alpha \phi+\phi_{0}$, and hence solves (3.5.1). Since $h^{\vee}$ has the delta function as a factor, $\left|f^{\vee}\right| \bar{j}^{-\alpha} \cdot h^{\vee}$ solves (3.5.2).

Lemma 4.12. If $\alpha \in A_{-}=A_{-}(b)$, then

$$
\left.\Gamma\left(V^{\vee}(\mathbb{R}), \underline{\operatorname{Hom}}_{D}\left(\mathscr{F}\left(D f^{\alpha}\right), \mathscr{B}\right)\right)\right)=\Gamma\left(\mathcal{Q}^{\vee}(\mathbb{R}), \underline{\operatorname{Hom}}_{D}\left(\mathscr{F}\left(D f^{\alpha}\right), \mathscr{B}\right)\right),
$$

and their dimension is equal to $l$.

(See (1.6) for $b=b(s),(2.3 .6)$ for $A_{ \pm}=A_{ \pm}(b)$, and $l$ for (4.7).)

Proof. We have

$$
\begin{aligned}
K: & =R \overline{\operatorname{Hom}}_{D}\left(\mathscr{F}\left(D f^{\alpha}\right), \mathscr{B}\right) \\
& \simeq R \overline{\operatorname{Hom}}_{D}\left(\mathscr{F}\left(D f^{\alpha}\right), R \Gamma_{V^{\vee}(R)} \mathcal{O}^{a n}\right)[n] \\
& =R \Gamma_{V^{\vee}(R)} \operatorname{Sol}\left(\mathscr{F}\left(D f^{\alpha}\right)\right)[n] \\
& =R \Gamma_{V^{\vee}(R)} R j_{*}^{\vee} i_{*}^{\vee}\left(L^{\vee}(-\alpha) \otimes H^{\vee}\right)[m], \quad \text { by }(3.23,(4)) .
\end{aligned}
$$

Since $\Gamma_{\left(V^{v}-\Omega^{v}\right)(\boldsymbol{R})} \cdot \Gamma_{V^{v}(\boldsymbol{R})^{\circ}} j_{*}^{v}=0, R \Gamma_{\left(\boldsymbol{V}^{v}-\Omega^{v}\right)(\boldsymbol{R})} K=0$. Hence $K=R \Gamma_{\Omega^{v}(\boldsymbol{R})} K$ and 


$$
\begin{aligned}
& R \Gamma\left(V^{\vee}(\boldsymbol{R}), R \underline{\operatorname{Hom}}_{D}\left(\mathscr{F}\left(D f^{\alpha}\right), \mathscr{B}\right)\right) \\
& \quad=R \Gamma\left(\mathscr{Q}^{\vee}(\boldsymbol{R}), R \underline{\operatorname{Hom}_{D}}\left(\mathscr{F}\left(D f^{\alpha}\right), \mathscr{B}\right)\right) .
\end{aligned}
$$

By comparing the spectral sequences determined by the both sides of (4.12.2), we get the first assertion. By (4.12.1),

$$
\begin{aligned}
R & \Gamma\left(\Omega^{\vee}(\boldsymbol{R}), R \underline{\operatorname{Hom}}_{D}\left(\mathscr{F}\left(D f^{\alpha}\right), \mathscr{B}\right)\right) \\
& \simeq R \Gamma\left(\Omega^{\vee}(\boldsymbol{R}), R \Gamma_{\Omega^{\vee}(\boldsymbol{R})} i_{*}^{\vee}\left(L^{\vee}(-\alpha) \otimes H^{\vee}\right)\right)[m] \\
& =\bigoplus_{j=1}^{i} R \Gamma\left(\Omega_{j}^{\vee}, i_{\boldsymbol{R}^{k}}^{\vee} R \Gamma_{O_{1}^{\vee}(\boldsymbol{R})}\left(L^{\vee}(-\alpha) \otimes H^{\vee}\right)\right)[m] .
\end{aligned}
$$

Here we denote the injection $O_{1}^{\vee}(\mathbb{R}) \rightarrow \Omega^{\vee}(\mathbb{R})$ by $i_{\boldsymbol{R}}^{\vee}$. Since $L^{\vee}(-\alpha) \otimes H^{\vee}$ is isomorphic to the constant sheaf $\mathbb{C}$ in a neighbourhood of $\Omega_{j}^{\vee} \cap O_{1}^{\vee}$ in $O_{1}^{\vee}(\mathbb{C})$,

$$
R \Gamma_{O_{1}^{\vee}(R)}\left(L^{\vee}(-\alpha) \otimes H^{\vee}\right) \simeq \mathbb{C}[-m]
$$

(Recall that $m=\operatorname{dim} O_{1}^{\vee}$ ). Hence (4.12.3) is isomorphic to

$$
\bigoplus_{j=1}^{l} R \Gamma\left(\Omega_{j}^{\vee}, i_{R^{*}}^{\vee} \mathbb{C}\right)=\bigoplus_{j=1}^{l} R \Gamma\left(\Omega_{j}^{\vee} \cap O_{1}^{\vee}, C\right),
$$

whose $H^{0}$ is of dimension $l$. (Recall that $\Omega_{j}^{\vee} \cap O_{1}^{\vee}(1 \leq j \leq l)$ are the connected components of $O_{1}^{\vee}(\mathbb{R})$.)

As a consequence of (4.11) and (4.12), we get the following lemma.

Lemma 4.13. If $\alpha \in A_{-}(b)$, every hyperfunction solution of $\mathscr{F}\left(D f^{\alpha}\right)$ on $\Omega^{\vee}(\boldsymbol{R})$ can be uniquely extended to a solution on $V^{\vee}(\mathbb{R})$. Denote the extension of $\left|f^{\vee}\right|_{j}^{-\alpha} h^{\vee}$ to a solution on $V^{\vee}(\mathbb{R})$ by the same letter. Then $\left\{\left|f^{\vee}\right| \bar{j}^{-\infty} h^{\vee}\right.$ $(1 \leq j \leq l)\}$ gives a basis of the vector space of the hyperfunction solutions of $\mathscr{F}\left(D f^{\alpha}\right)$ on $V^{\vee}(\boldsymbol{R})$.

4.14. Define a real analytic function $|f|_{j}^{\alpha}$ on $\Omega(\mathbb{R})$ by

$$
|f|_{j}^{\alpha}= \begin{cases}|f|^{\alpha}, & \text { on } \Omega_{j} \\ 0, & \text { on } \Omega(\mathbb{R})-\Omega_{j} .\end{cases}
$$

Obviously $|f|_{j}^{\alpha}(1 \leq j \leq l)$ are hyperfunction solutions of $D f^{\alpha}$. By the same argument as above, we get the following lemma.

Lemma 4.15. If $\alpha \in A_{+}(b)$, every hyperfunction solution of $D f^{\alpha}$ on $\boldsymbol{Q}(\boldsymbol{R})$ can be uniquely extended to a solution on $V(\mathbb{R})$. Denote the extension of $|f|_{j}^{\alpha}$ to a solution on $V(\boldsymbol{R})$ by the same letter. Then $\left\{|f|_{j}^{\alpha}(1 \leq j \leq l)\right\}$ gives a basis of the vector space of the hyperfunction solutions of $D f^{\infty}$ on $V(\mathbb{R})$. 
Remark 4.16. The above lemma holds for any polynomial $f$, if $b(s)$ is replaced by $B(s)$ (cf. (2.5.1)). It is also possible to get an analytic version.

4.17. Let $S=S(V)$ be the set of $C^{\infty}$-functions $\varphi(x)$ on $V(\mathbb{R})$ such that $\mathbb{P} \varphi(x)$ is bounded for any $P \in D(V)$, and $\mathcal{S}^{\prime}=\mathcal{S}^{\prime}(V)$ its dual. An element $u=u(x)$ is called a tempered distribution on $V(\mathbb{R})$. Cf. [4]. Denote the value of $u$ at $\varphi \in \mathcal{S}$ by $\int u(x) \varphi(x) d x$. The $D(V)$-module structure of $\mathcal{S}^{\prime}$ is defined by $\int P u(x) \circ \varphi(x) d x=\int u(x) \circ P^{*} \varphi(x) d x$ (cf. (2.1.2)). Let $A$ be a domain in $\mathbb{C}$. A family $u_{\alpha}(x)(\alpha \in A)$ of tempered distributions is a said to be holomorphic (resp. meromorphic) in $\alpha$, if for any $\varphi(x) \in \mathcal{S}, \alpha \rightarrow \int u_{\alpha}(x) \varphi(x) d x$ is holomorphic (resp. meromorphic). If for any $\varphi(x) \in \mathcal{S}, \int u_{\alpha}(x) \varphi(x) d x$ has a pole of order $\leq \rho$ at $\alpha=\alpha_{0}$, we say that $u_{\alpha}(x)$ has a pole of order $\leq \rho$ at $\alpha=\alpha_{0}$. We can naturally define a pole of order $\rho$ etc. Define a Fourier transform $\mathscr{F}(\varphi)$ of $\varphi \in \mathcal{S}$ by

$$
\mathscr{I}(\varphi)(y)=\int_{V(\mathbb{R})} \varphi(x) e^{-v \overline{-1}\langle x \mid y\rangle} d x,
$$

where $d x$ is an Euclidean measure of $V(\mathbb{R})$. Define the Fourier transform $\mathscr{F}(u)$ of $u \in \mathcal{S}^{\prime}(V)$ by

$$
\int \mathscr{F}(u)(y) \varphi(y) d y=\int u(x) \mathscr{F}(\varphi)(x) d x
$$

for $\varphi \in S\left(V^{\vee}\right)$. Then

$$
\mathscr{H}(P u)=\mathscr{F}(P) \mathscr{F}(u)
$$

(cf. 2.7.1).

4.18. Let

$$
f_{j}^{\alpha}= \begin{cases}|f|_{j}^{\alpha}, & \text { if } f>0 \text { in } \Omega_{j} \\ \exp (\alpha \pi \sqrt{-1})|f|_{j}^{\alpha}, & \text { if } f<0 \text { in } \Omega_{j},\end{cases}
$$

and

$$
f_{j}^{\vee-\alpha}= \begin{cases}\left|f^{\vee}\right|_{j}^{-\alpha}, & \text { if } f^{\vee}>0 \text { in } \Omega_{j}^{\vee} \\ \exp (-\alpha \pi \sqrt{-1})\left|f^{\vee}\right|_{j}^{-\alpha} h, & \text { if } f^{\vee}<0 \text { in } \Omega_{j}^{\vee} .\end{cases}
$$

If the real part of $\alpha \in \mathbb{C}$ is non-negative, the real analytic function $f_{j}^{\alpha}$ on $\Omega(\mathbb{R})$ can be extended to a continuous function on $V(\mathbb{R})$, which we shall consider as a tempered distribution on $V(\mathbb{R})$. Then $f_{j}^{a}$ is holomorphic in $\{\alpha \mid \operatorname{Re}(\alpha)>0\}$. If $\operatorname{Re}(\alpha)>-m, f_{j}^{\alpha}=(b(\alpha+m-1) \cdots b(\alpha))^{-1} f^{\vee}(\partial)^{m} f_{j}^{\alpha+m}$. Hence $f_{j}^{a}$ is meromorphic on the whole complex plane $\mathbb{C}$, and holomorphic on $A_{+}=$ $A_{+}(b)$. (Cf. (2.3.6).) 
Let $A=A(V), D=D(V)$ and $P \in D_{k}[s]$ (cf. (2.1.1)). If $P(s) f^{s}=a(s) f^{s-k}$ with $a(s) \in A[s]$. Then $P(\alpha) f_{j}^{\alpha}=a(\alpha) f_{j}^{\alpha-k}$ on $\Omega$. If $\operatorname{Re}(\alpha-k)>0$, this equality holds on $V(\mathbb{R})$ as usual functions. Hence it holds also as tempered distributions. By an analytic continuation, the equality holds as meromorphic families of tempered distributions. Hence if $P f^{\alpha}=0$ in $D f^{\alpha}$ (cf. (2.3.1)), then $P f_{j}^{\alpha}=0$. In other words, $f_{j}^{\alpha}$ is a solution of $D f^{\alpha}$. By (4.17.1) and (2.7.1), $\mathscr{F}\left(f_{j}^{\alpha}\right)$ is a solution of $\mathscr{F}\left(D f^{\alpha}\right)$.

Theorem 4.19. (1) By an analytic continuation, $f_{j}^{\vee-\infty} h^{\vee}$ is extended to a single-valued meromorphic family of tempered distributions on the whole complex plane $\mathbb{C}$ which is holomorphic in $A_{-}(b)$.

(2) There exist meromorphic functions $c_{i j}(\alpha)(1 \leq i, j \leq l)$ such that

$$
\mathscr{F}\left(f_{i}^{\alpha}\right)=\sum_{j=1}^{l} c_{i j}(\alpha) f_{j}^{\vee-\alpha} h^{\vee} \quad(1 \leq i \leq l),
$$

and which are holomorphic in $A_{+}(b)$.

(See (4.13) and (4.18) for $f_{j}^{\vee-\alpha} h^{\vee},(4.15)$ and (4.18) for $f_{i}^{\alpha}, b$ for (1.6), $A_{ \pm}(b)$ for (2.3.6) and $\mathscr{F}$ for (4.17).)

Proof. Let $\alpha \in A_{+} \cap A_{-}$. As is shown in (4.18), $\mathscr{F}\left(f_{i}^{a}\right)(1 \leq i \leq l)$ are solutions of $\mathscr{F}\left(D f^{\infty}\right)$ on $V^{\vee}(\mathbb{R})$, which are linearly independent. By (4.12), they give a basis of the space of global hyperfunction solutions of $\mathscr{F}\left(D f^{\alpha}\right)$. By (4.13), $f_{j}^{\vee-\infty} h^{\vee}(1 \leq j \leq l)$ also give a basis of the same space. Hence there exist functions $c_{i j}(\alpha)(1 \leq i, j \leq l)$ on $A_{+} \cap A_{-}$such that

$$
\mathscr{F}\left(f_{i}^{a}\right)=\sum_{j=1}^{l} c_{i j}(\alpha) f_{j}^{\vee-\alpha} h^{\vee},
$$

and $\operatorname{det}\left(c_{i j}(\alpha)\right) \neq 0$. Since $f_{j}^{\vee-\alpha} h^{\vee}$ can be expressed as linear combinations of tempered distributions $\mathscr{L}\left(f_{i}^{\alpha}\right), f_{j}^{\vee-\alpha} h^{\vee}$ are also tempered distributions. If the support of $\varphi(y) \in \mathcal{S}\left(V^{\vee}\right)$ is contained in $\Omega_{j}^{\vee}$,

$$
c_{i j}(\alpha)=\frac{\int \mathscr{F}_{i}\left(f_{i}^{\alpha}\right)(y) \cdot \varphi(y) d y}{\int\left(f_{j}^{\vee-\alpha} h^{\vee}\right)(y) \cdot \varphi(y) d y} .
$$

Suitably choosing $\varphi(y)$, the denominator of the right member becomes an entire function which does not take the value zero. As is shown in (4.18), $f_{i}^{a}$ are meromorphic on the whole complex plane and holomorphic in $A_{+}(b)$. Hence $c_{i j}(\alpha)$ are also meromorphic on $\mathbb{C}$ and holomorphic in $A_{+}(b)$. Thus we get (2). In the same time, it follows that $f_{j}^{\vee-\alpha} h^{\vee}$ are meromorphic on $C$. Let $\alpha_{0} \in A_{-}$ and assume that $f_{j}^{\vee-\infty} h^{\vee}$ has a pole of order $\rho(>0)$ at $\alpha=\alpha_{0}$. Then $u=u(y)=$ $\left.\left(\alpha-\alpha_{0}\right)^{\rho} \cdot f_{j}^{\vee-\alpha} h^{\vee}\right|_{\alpha=\alpha_{0}}$ gives a solution of $\mathscr{F}\left(D f^{\alpha}\right)$, which is a tempered distri- 
bution. If the support of $\varphi(y) \in \mathcal{S}\left(V^{\vee}\right)$ is contained in $\Omega_{j}^{\vee}, \int \varphi(y) u(y) d y=0$. Hence the support of $u(y)$ is contained in $\left(V^{\vee}-\Omega^{\vee}\right)(\mathbb{R})$. Hence $u(y)$ is not a linear combination of $f_{j}^{\vee-\alpha_{0}} h^{\vee}$. This consequence contradicts (4.13). Hence $f_{j}^{-\alpha} h^{\vee}$ is holomorphic in $A_{-}$.

Remark 4.20. We can determine $c_{i j}(\alpha)$ to the same extent as in [24], [28], [30] by the same argument.

Remark 4.21. The theory of prehomogeneous vector spaces is originated by M. Sato in 1961 in order to give a testing ground for investigating a 'general theory of linear differential equations'. Such a 'general theory' is realized as the theory of $D$-modules, mainly by $\mathbb{M}$. Sato, T. Kawai and M. Kashiwara. We can see in [25] a deep relation between the theory of $D$-modules (especially, their microlocal analysis) and the theory of prehomogeneous vector spaces.

The original theory of prehomogeneous vector spaces is based on the invariance with respect to a very large group. An invariance with respect to a connected Lie group is nothing but an invariance with respect to the corresponding Lie algebra, which is also expressed as a system of linear differential equations of first order. This system is $N^{\prime}(\alpha)=D v_{\alpha}^{\prime}$ given by (3.24.1). Thus we can also say that the original theory of prehomogeneous vector spaces is based on the system $N^{\prime}(\alpha)$ of linear differential equations of first order.

Since the defining equations of $N^{\prime}(\alpha)$ are explicitly given, it is easy to determine its Fourier transform. But for a further investigation, $N^{\prime}(\alpha)$ seems not to be good. For example, it seems inevitable to consider $N(\alpha)=D f^{\alpha}$ given in (2.3.1) instead of $N^{\prime}(\alpha)$ to get such consequences as (4.12), (4.13) and (4.15). If we assume the regularity condition, the difference between $N^{\prime}(\alpha)$ and $N(\alpha)$ is not so large and sometimes negligible. But if we do not assume the regularity condition, as is seen from (3.24.2), the difference is not negligible at all. Thus it becomes essential to investigate $N(\alpha)=D f^{\alpha}$, especially to determine its Fourier transform. For this purpose, we needed the theory of $D$-modules, which is, in a sense, a fruit of the original theory of prehomogeneous vector spaces. (It would be worth noting here that $N(\alpha)$ is the main object of [25]. Although it might seem that $N^{\prime}(\alpha)$ is exclusively studied there, $N(\alpha)$ comes into the study as a study of $N^{\prime}(\alpha)$ on a good Lagrangian.)

\section{References}

[1] Björk, J.-E., Rings of differential operators, North-Holland, Amsterdam-New YorkOxford, 1979. 
[2] Bopp, N. and Rubenthaler, H., Fonction zêta associée à la série principale sphérique de certains espaces symétriques, C.R.Acad. Sci. Paris, 310 (1990), 505-508.

[3] Bourbaki, N., Algèbre commutative, Hermann, Paris, 1961.

[4] Gel'fand, I.M. and Shilov, G.E., Generalized functions, vol. 1, Academic Press, New York-London, 1964.

[ 5] Gyoja, A., Representations of reductive group schemes, Tsukuba J. Math., 15 (1991), 335-346.

[6] - A counterexample in the theory of prehomogeneous vector spaces, Proc. Japan Acad., 66 (1990), 26-27.

[ 7 ] - Invariants, nilpotent orbits and prehomogeneous vector spaces, J. Algebra, 142 (1991), 210-232.

[8] - Vector valued invariants of prehomogeneous vector spaces, J. Math. Soc. Japan, 43 (1991), 117-131.

[9] - Theory of prehomogeneous vector spaces, (note by T. Kimura in Japanese), Surikaiseki Kenkyusho Kokyuroku, 718 (1990).

[10] Hironaka, H., Resolution of singularities of an algebraic variety over a field of characteristic zero I, II, Ann. of Math., 79 (1964), 109-326.

[11] Hotta, R., Introduction to D-modules, The Institute of Mathematical Sciences, Madras, 1987.

[12] Hotta, R. and Kashiwara, M., The invariant holonomic system on a semisimple Lie algebra, Invent. Math., 75 (1984), 327-358.

[13] Kashiwara, M., b-Functions and holonomic systems, Invent. Math., 38 (1976), 33-53.

[14] - On the holonomic systems of linear differential equations, II, Invent. Math., 49 (1978), 121-135.

[15] —, Systems of microdifferential equations, Progress in Mathematics, 34, Birkhäuser, Boston-Basel-Stuttgart. 1983.

[16] Kashiwara, M. and Kawai, T., On the characteristic variety of a holonomic system with regular singularities, Adv. in Math., 34 (1979), 163-184.

[17] Kashiwara, M., Kawai, T. and Kimura, T., Foundaitons of algebraic analysis, Princeton University Press, Princeton, 1986.

[18] Kawanaka, N., Generalized Gelfand-Graev representations of exceptional simple algebraic groups over a finite field I, Invent. Math., 84 (1986), 575-616.

[19] Luna, D., Sur les orbites fermées des groupes algèbriques réductifs, Invent. Math., 16 (1972), 1-5.

[20] Matsushima, Y., Espaces homogènes de Stein des groupes de Lie complexes, Nagoya Math. J., 16 (1960), 205-218.

[21] Mumford, D., Geometric invariant theory, Springer Verlag, Berlin-Heidelberg-New York, 1965.

[22] Popov, V.L., Stability criteria for the action of a semisimpie group on a factorial manifold, Math. USSR-Izv., 4 (1970), 527-535.

[23] Rubenthaler, H. and Schiffmann, G., Opérateurs différentiels de Shimura et espaces préhomogènes, Invent. Math., 90 (1987), 409-442.

[24] Sato, M., Theory of prehomogeneous vector spaces (note by T. Shintani in Japanese), Sugaku no Ayumi, 15 (1970), 85-157.

[25] Sato, M., Kashiwara, M., Kimura, T. and Oshima, T., Micro-local analysis of prehomogeneous vector spaces, Invent. Math., 62 (1980), 117-179.

[26] Sato, M., Kawai, T. and Kashiwara, M., Microfunctions and pseudo-differential equations, Proc. Katata Conf., Lecture Notes in Math., Springer, 287 (1973), 263-529.

[27] Sato, M. and Kimura, T., A classification of irreducible prehomogeneous vector spaces and their relative invariants, Nagoya Math. J., 65 (1977), 1-155.

[28] Sato, M. and Shintani, T., On zeta functions associated with prehomogeneous vector 
spaces, Ann. of Math., 100 (1974), 131-170.

[29] Serre, J.-P., Algèbre locale-multiplicités, Lecture Notes in Math., Springer, $\mathbb{1} 1$ (1965).

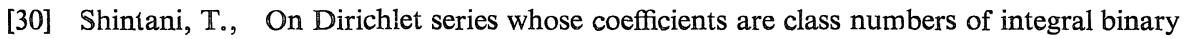
cubic forms, J. Math. Soc. Japan, 24 (1972), 132-188.

[31] Springer, T.A. and Steinberg, R., Conjugacy classes, Seminar on algebraic groups and related finite groups, Lecture Notes in Muth., Springer, 131 (1970).

[32] Steinberg, R., Conjugacy classes in algebraic groups, Lecture Notes in Math., Springer, 366 (1974).

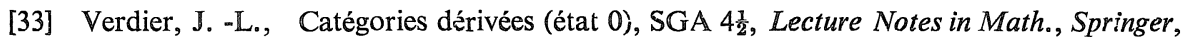
569 (1977). 